\title{
On the Enumeration of Irreducible Polynomials over GF $(q)$ with Prescribed Coefficients
}

\author{
Robert Granger \\ Secure Systems Research Group \\ Department of Computer Science \\ University of Surrey \\ United Kingdom \\ r.granger@surrey.ac.uk
}

Dedicated to the memory of Oonagh Ni Chéileachair.

Sonas ort.

\begin{abstract}
We present an efficient deterministic algorithm which outputs exact expressions in terms of $n$ for the number of monic degree $n$ irreducible polynomials over $\mathbb{F}_{q}$ of characteristic $p$ for which the first $l<p$ coefficients are prescribed, provided that $n$ is coprime to $p$. Each of these counts is $\frac{1}{n}\left(q^{n-l}+\mathcal{O}\left(q^{n / 2}\right)\right)$. The main idea behind the algorithm is to associate to an equivalent problem a set of Artin-Schreier curves defined over

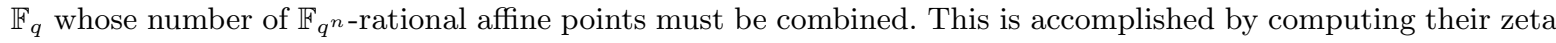
functions using a $p$-adic algorithm due to Lauder and Wan. Using the computational algebra system Magma one can, for example, compute the zeta functions of the arising curves for $q=5$ and $l=4$ very efficiently, and we detail a proof-of-concept demonstration. Due to the failure of Newton's identities in positive characteristic, the $l \geq p$ cases are seemingly harder. Nevertheless, we use an analogous algorithm to compute example curves for $q=2$ and $l \leq 7$, and for $q=3$ and $l=3$. Again using Magma, for $q=2$ we computed the relevant zeta functions for $l=4$ and $l=5$, obtaining explicit formulae for these open problems for $n$ odd, as well as for subsets of these problems for all $n$, while for $q=3$ we obtained explicit formulae for $l=3$ and $n$ coprime to 3 . We also discuss some of the computational challenges and theoretical questions arising from this approach in the general case and propose some natural open problems.
\end{abstract}

Keywords: Irreducible polynomials, prescribed coefficients, prescribed traces, Artin-Schreier curves, zeta functions, binary Kloosterman sums.

MSC: 12Y05, 11T06, 11Y16, 11G20.

\section{Introduction}

For $q=p^{r}$ a prime power let $\mathbb{F}_{q}$ denote the finite field of $q$ elements, and let $I_{q}(n)$ denote the number of monic irreducible polynomials in $\mathbb{F}_{q}[x]$ of degree $n$. A classical result due to Gauss [19, pp. 602-629] states that

$$
I_{q}(n)=\frac{1}{n} \sum_{d \mid n} \mu(d) q^{n / d}
$$

A natural problem is to determine the number of monic irreducible polynomials in $\mathbb{F}_{q}[x]$ of degree $n$ for which certain coefficients are prescribed, which we refer to in general as the prescribed coefficients problem. As Panario has stated [43, p. 115], "The long-term goal here is to provide existence and counting results for irreducibles with any number of prescribed coefficients to any given values. This goal is completely out of reach at this time. Incremental steps seem doable, but it would be most interesting if new techniques were introduced to attack these problems."

Regarding existence, building upon work of Bourgain [6] and Pollack [44], the best result to date is due to $\mathrm{Ha}$ [23], who in 2016 proved that there exists a monic irreducible polynomial in $\mathbb{F}_{q}[x]$ of degree $n$ with up to $\lfloor(1 / 4-\epsilon) n\rfloor$ coefficients prescribed in any positions, for any $\epsilon>0$ and $q$ sufficiently large.

In contrast to Ha's important progress towards the above long-term goal on the existence side, the corpus of counting results is far less well developed. Nearly all such research has focused on the subproblem of determining the number of monic irreducible polynomials in $\mathbb{F}_{q}[x]$ of degree $n$ for which the first $l$ 
coefficients have the prescribed values $t_{1}, \ldots, t_{l}$, which we denote by $I_{q}\left(n, t_{1}, \ldots, t_{l}\right)$. Although asymptotics for such subproblems have been obtained by Cohen [10], very few exact results are known. In 1952 Carlitz gave formulae for $I_{q}\left(n, t_{1}\right)$ [7], while in $1990 \mathrm{Kuz}$ 'min gave formulae for $I_{q}\left(n, t_{1}, t_{2}\right)$ [32,33]; Cattell et al. later reproduced Kuz'min's results for the base field $\mathbb{F}_{2}$, in 1999 [8]. In 2001 the three coefficient case $I_{2}\left(n, t_{1}, t_{2}, t_{3}\right)$ was solved, by Yucas and Mullen for $n$ even [57] and by Fitzgerald and Yucas for $n$ odd [17]. Formulae for $I_{2^{r}}\left(n, t_{1}, t_{2}\right)$ for all $r \geq 1$ were given again in 2013 by Ri et al. [45]. Most recently, in 2016 Ahmadi et al. gave formulae for $I_{2^{r}}(n, 0,0,0)$ for all $r \geq 1$ [3].

Rather than study the above subproblem instances directly, the papers $[8,57,17,45,3]$ all study a set of equivalent problems, namely counting the number of elements of $\mathbb{F}_{q^{n}}$ with correspondingly prescribed traces, which we refer to in general as the prescribed traces problem. In particular, for $a \in \mathbb{F}_{q^{n}}$ the characteristic polynomial of $a$ with respect to the extension $\mathbb{F}_{q^{n}} / \mathbb{F}_{q}$ is defined to be:

$$
\prod_{i=0}^{n-1}\left(x-a^{q^{i}}\right)=x^{n}-T_{1}(a) x^{n-1}+T_{2}(a) x^{n-2}-\cdots+(-1)^{n-1} T_{n-1}(a) x+(-1)^{n} T_{n}(a),
$$

with $T_{l}: \mathbb{F}_{q^{n}} \rightarrow \mathbb{F}_{q}, 1 \leq l \leq n$ the successive trace functions

$$
\begin{aligned}
T_{1}(a) & =\sum_{i=0}^{n-1} a^{q^{i}}, \\
T_{2}(a) & =\sum_{0 \leq i_{1}<i_{2} \leq n-1} a^{q^{i_{1}}+q^{i_{2}}}, \\
T_{3}(a) & =\sum_{0 \leq i_{1}<i_{2}<i_{3} \leq n-1} a^{q^{i_{1}}+q^{i_{2}}+q^{i_{3}}}, \\
& \vdots \\
T_{l}(a) & =\sum_{0 \leq i_{1}<\cdots<i_{l} \leq n-1} a^{q^{i_{1}}+\cdots+q^{i_{l}}}, \\
& \vdots \\
T_{n}(a) & =a^{1+q+q^{2}+\cdots+q^{n-1}} .
\end{aligned}
$$

For any $n \geq l$ and $t_{1}, \ldots, t_{l} \in \mathbb{F}_{q}$, let $F_{q}\left(n, t_{1}, \ldots, t_{l}\right)$ denote the number of elements $a \in \mathbb{F}_{q^{n}}$ for which $T_{1}(a)=t_{1}, \ldots, T_{l}(a)=t_{l}$. If for a given $q=p^{r}$ and $l \geq 1$ one determines $F_{q}\left(n, t_{1}, \ldots, t_{l}\right)$ for all $\left(t_{1}, \ldots, t_{l}\right) \in$ $\left(\mathbb{F}_{q}\right)^{l}$, then an application of the multinomial theorem and a generalised Möbius inversion-type argument gives $I_{q}\left(n, t_{1}, \ldots, t_{l}\right)$ for all $\left(t_{1}, \ldots, t_{l}\right) \in\left(\mathbb{F}_{q}\right)^{l}$, and vice versa, hence the equivalence. The formulae for the equivalence follow from the approach of Miers and Ruskey [40], extending the subcases already proven for binary fields $[8,57,17,45,3]$. For our main case of interest for which $l<p$, each $I_{q}\left(n, t_{1}, \ldots, t_{l}\right)$ can be expressed in terms of only one $F_{q}\left(n, t_{1}^{\prime}, \ldots, t_{l}^{\prime}\right)$, and vice versa, see $\S 2.4$.

As well as proving formulae for $I_{2^{r}}(n, 0,0,0)$ for all $r \geq 1$, the work [3] also explained an intriguing phenomenon, which is that the formulae for $F_{2}\left(n, t_{1}, t_{2}\right)$ and $F_{2}\left(n, t_{1}, t_{2}, t_{3}\right)$ proven in [8] and [57,17] depend on $n \bmod 8$ and on $n \bmod 24$, respectively. In particular, by Fourier analysing the formulae the present

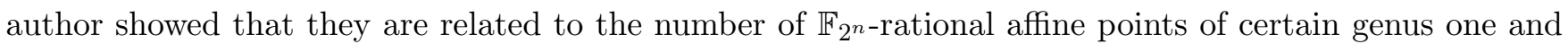
two supersingular curves defined over $\mathbb{F}_{2}$. A simple argument gave a new derivation of the formulae for the $t_{1}=0$ cases and the $n$ odd cases, and since the curves featured are supersingular their normalised Weil numbers are roots of unity, which explains the observed periodicity.

In this paper we greatly extend the curve-based approach of [3] in order to prove the following theorem. Let $\overline{\mathbb{Q}}$ be an algebraic closure of $\mathbb{Q}$ and let $\overline{\mathbb{Z}}$ be the integral closure of $\mathbb{Z}$ in $\overline{\mathbb{Q}}$.

Theorem 1. Let $q=p^{r}, l<p$ and let

$$
N= \begin{cases}\left(q^{l}(q l-q-l)+q\right) & \text { if } r=1 \\ \left(q^{l}(q l-q-l)+q\right)(p-1) & \text { if } r>1\end{cases}
$$


Then for each $\bar{n} \in\{1, \ldots, p-1\}$ there exist $\omega_{1}, \ldots, \omega_{N} \in \overline{\mathbb{Z}}$ (not necessarily distinct), which are all of norm $\sqrt{q}$, such that for all $\left(t_{1}, \ldots, t_{l}\right) \in\left(\mathbb{F}_{q}\right)^{l}$ there exist explicitly determined $v_{1}, \ldots, v_{N} \in\{0, \pm 1\}$ such that for all $n \equiv \bar{n}(\bmod p)$ with $n \geq l$ one has

$$
F_{q}\left(n, t_{1}, \ldots, t_{l}\right)=\frac{1}{q^{l}}\left(q^{n}+\frac{q-1}{p-1} \sum_{k=1}^{N} v_{k} \omega_{k}^{n}\right)=q^{n-l}+\mathcal{O}\left(q^{n / 2}\right) .
$$

Moreover, there exists an explicit deterministic algorithm which for each $\bar{n}$ computes $\omega_{1}, \ldots, \omega_{N}$ in $q^{l}$. $\tilde{\mathcal{O}}\left(l^{5} p^{4}\right)$ bit operations when $r=1$, and $q^{l+1} \cdot \tilde{\mathcal{O}}\left(l^{5} p^{4} r^{3}\right)$ bit operations when $r>1$.

Here we use the soft-oh notation $\tilde{\mathcal{O}}$, which ignores factors which are logarithmic in the argument. Note that Theorem 1 implies an asymptotic equidistribution result for the prescribed traces problem, as one expects.

The main idea behind the algorithm is to associate to the prescribed traces problem a fibre product of $l$ Artin-Schreier curves defined over $\mathbb{F}_{q}$, whose number of $\mathbb{F}_{q^{n}}$-rational affine points solves the problem. In order to count this number we propose two methods. The first - which we refer to as the direct method - computes a single $F_{q}\left(n, t_{1}, \ldots, t_{l}\right)$ by replacing the fibre product by an intersection. However, this results in curves of relatively large genus for which we do not know of an efficient point counting algorithm. The second - which we refer to as the indirect, or 'batching' method-determines all of the $q^{l}$ counts $F_{q}\left(n, t_{1}, \ldots, t_{l}\right)$ simultaneously, by transforming these counting problems into a set of $q^{l}$ equivalent counting problems. All but one of the latter problems requires computing the zeta function of an ArtinSchreier curve defined over $\mathbb{F}_{q}$, which is accomplished using a $p$-adic algorithm due to Lauder and Wan [35]. While obtaining the relevant curves is immediate, determining their zeta functions is a fundamentally computational problem, so one should not expect to be able to simply write down general formulae for $F_{q}\left(n, t_{1}, \ldots, t_{l}\right)$. There may of course exist faster algorithms for determining the exact formulae than the approach we take, particularly when only one $F_{q}\left(n, t_{1}, \ldots, t_{l}\right)$ is required, but we emphasise that the one presented here constitutes the first algorithmic approach to solving the prescribed traces problem exactly, which therefore represents a shift in perspective with regard to its study.

Although the indirect method is very efficient for computing all $q^{l}$ counts $F_{q}\left(n, t_{1}, \ldots, t_{l}\right)$ simultaneously, a potential disadvantage is that the number $N$ of algebraic integers featuring in Theorem 1 may be larger than for the direct method, even accounting for multiplicities. There may, therefore, be some redundancy in the resulting formulae. Such redundancy can be eliminated with some postcomputation by identifying, for each $\bar{n}$, cancellations between linear combinations of $n$-th powers of the $\omega_{k}$ 's which are valid for all $n \equiv \bar{n}(\bmod p)$, see $\S 5$ for an example. One should therefore attempt to use the direct method first, and if it is not computationally feasible then use the indirect method, noting that some redundancy elimination may be required.

It should be no surprise that the formulae for $F_{q}\left(n, t_{1}, \ldots, t_{l}\right)$ arise from the number of points on curves. As noted by Voloch [53], a method used by Hayes [25], Hsu [27], Voloch himself and others to estimate $I_{q}\left(n, t_{1}, \ldots, t_{l}\right)$ relates these counts to the number of points over $\mathbb{F}_{q^{n}}$ of certain curves defined over $\mathbb{F}_{q}$ whose function fields are subfields of the so-called cyclotomic function fields. The equivalence to $F_{q}\left(n, t_{1}, \ldots, t_{l}\right)$ then implies that these counts are related to the number of points on curves as well.

The prescribed traces problem that we partially solve in this work is similar to a problem studied by Miers and Ruskey [39]. In particular, using combinatorial techniques, expressions were given for the number of strings over finite rings with prescribed symmetric function evaluations. However, while this problem is similar the techniques used do not seem applicable to the prescribed traces problem as we have defined it. More relevant to our case, but still seemingly inapplicable are the expressions given by Miers and Ruskey for the number of equivalence classes of aperiodic strings over finite rings with prescribed symmetric function evaluations, under rotation [40]. However, as already mentioned the formulae for the equivalence between the prescribed coefficients problem and the prescribed traces problem that we study follow easily from this work.

A fundamental limitation of our proposed algorithm is that it breaks for $l \geq p$ due to the failure of Newton's identities in positive characteristic. Nevertheless, by employing a slightly different but likely 
limited technique to bypass the $l<p$ constraint, we computed curves for $q=2, l \leq 7$ and $n$ odd whose

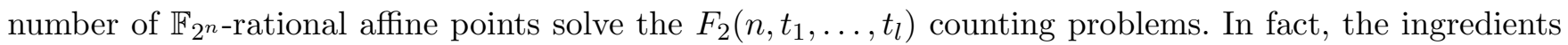
of our main algorithm were initially developed for $q=2$, since even the $l=4$ cases were open problems with the only previous result being an approximation to the counts [30,31] (there is however a small error in the transforms used, see $\S 4.6$ ). We used the computational algebra system Magma [5] to compute the corresponding zeta functions for $l=4$ and $l=5$, obtaining explicit formulae for these open problems for $n$ odd. Interestingly, for these cases the characteristic polynomial of the Frobenius endomorphism of the featured curves have factors that arise from ordinary - rather than supersingular - abelian varieties, and a simple argument implies that the set of formulae $\left\{F_{2}\left(n, t_{1}, t_{2}, t_{3}, t_{4}\right)\right\}_{n \geq 4}$ and $\left\{F_{2}\left(n, t_{1}, t_{2}, t_{3}, t_{4}, t_{5}\right)\right\}_{n \geq 5}$ for each $\left(t_{1}, \ldots, t_{4}\right)$ and $\left(t_{1}, \ldots, t_{5}\right)$ respectively, can not be periodic in $n$, as in the $l=2$ and $l=3$ cases. This perhaps explains why there had been little progress in the four coefficients problem for the past 15 years, since there is not a finite set of cases to enumerate. We also give formulae for a subset of $F_{2}\left(n, t_{1}, t_{2}, t_{3}, t_{4}\right)$ and $F_{2}\left(n, t_{1}, t_{2}, t_{3}, t_{4}, t_{5}\right)$ which are valid for all $n \geq 4$ and $n \geq 5$, respectively. Somewhat surprisingly, the formulae for $F_{2}\left(n, 0,0,0, *, t_{5}\right)$ for $n \geq 5$ - where the asterisk means that we do not mind what the value of the fourth trace function is - are periodic in $n$ with period 120. It is therefore conceivable that these formulae could have been discovered without using our curve-based approach, although no doubt far less efficiently. The method for $q=2$ extends to one for any $q=2^{r}$ and $l \leq 7$, and also extends to $q=3^{r}$ and $l \leq 3$, which features non-supersingular Weil numbers for just three prescribed coefficients when $r=1$.

The sequel is organised as follows. In $\S 2$ we present our main algorithm for arbitrary $q=p^{r}, l<p$ and $n$ coprime to $p$, and present details of a very efficient proof-of-concept demonstration for $q=5$ and $l=4$. In $\S 3$ we present direct and indirect methods for solving the prescribed traces problem for $q=2$ and $n$ odd and discuss its possible limitations. Then in $\S 4$ we apply these methods to compute curves for $l \leq 7$, some for $n$ odd and some for all relevant $n$, provide explicit formulae for $l=4$ and $l=5$, and for $l=4$ detail a connection to binary Kloosterman sums and provide the correct transform from the prescribed traces problems to the prescribed coefficients problem. In $\S 5$ we present some formulae for the $q=l=3$ case. Finally, in $\S 6$ we discuss some of the computational challenges and theoretical questions arising from our approach in the general case and propose some natural open problems.

We assume the reader is familiar with curves and their zeta functions. Should they not be, the relevant definitions may be found in $\S 3$ of the antecedent to this work [3], which we recommend to the reader. The code used for all interesting computations performed with Magma and Maple [1] is openly available from https://github.com/robertgranger/CountingIrreducibles, with the relevant files indicated in footnotes in the text.

\section{The Main Algorithm}

In this section we present an algorithm for solving the prescribed traces problem - and thus the prescribed coefficients problem - exactly for any prime power $q=p^{r}$, any $1 \leq l<p$ and any $n \geq l$ coprime to $p$. The input traces whose values are prescribed are $T_{1}, \ldots, T_{l}$, i.e., the problem is to compute

$$
F_{q}\left(n, t_{1}, \ldots, t_{l}\right)=\#\left\{a \in \mathbb{F}_{q^{n}} \mid T_{1}(a)=t_{1}, \ldots, T_{l}(a)=t_{l}\right\}
$$

for any $t_{1}, \ldots, t_{l} \in \mathbb{F}_{q}$. We begin with what we refer to as the direct method in $\S 2.1$ and present the indirect, or batching method in $\S 2.3$.

\subsection{Direct method}

We begin with the following extremely simple lemma, whose proof follows from [36, Theorem 2.25].

Lemma 1. (1) For $a \in \mathbb{F}_{q^{n}}$ the condition $T_{1}(a)=0$ is equivalent to $a=a_{1}^{q}-a_{1}$, for $q$ different $a_{1} \in \mathbb{F}_{q^{n}}$.

(2) For $a \in \mathbb{F}_{q^{n}}$ and $n \not \equiv 0(\bmod p)$, the condition $T_{1}(a)=t_{1}$ is equivalent to $a=a_{1}^{q}-a_{1}+t_{1} / n$, for $q$ different $a_{1} \in \mathbb{F}_{q^{n}}$. 
We now recall Newton's identities over $\mathbb{Z}$ (see e.g., [36, Theorem 1.75]) with indeterminates $\alpha_{1}, \ldots, \alpha_{n}$. Abusing notation slightly, we refer to the elementary symmetric polynomials in $\alpha_{1}, \ldots, \alpha_{n}$ as $T_{1}(\alpha), T_{2}(\alpha), \ldots$, and to the power sum symmetric polynomials $\alpha_{1}^{j}+\cdots+\alpha_{n}^{j}$ as $T_{1}\left(\alpha^{j}\right)$ for $j \geq 1$, i.e., we work in the ring of symmetric functions, suppressing the dependence on $n$. We use the convention that $T_{0}(\alpha)=1$.

Lemma 2. For all $k \geq 1$ and $n \geq k$ we have

$$
k T_{k}(\alpha)=\sum_{j=1}^{k}(-1)^{j-1} T_{k-j}(\alpha) T_{1}\left(\alpha^{j}\right) .
$$

Letting $\alpha=a$ and substituting $T_{j}(a)=t_{j}$ for $1 \leq j \leq k$, Eq. (3) becomes

$$
\begin{aligned}
k t_{k} & =\sum_{j=1}^{k}(-1)^{j-1} t_{k-j} T_{1}\left(a^{j}\right) \\
& =(-1)^{k-1} T_{1}\left(a^{k}\right)+\sum_{j=1}^{k-1}(-1)^{j-1} t_{k-j} T_{1}\left(a^{j}\right),
\end{aligned}
$$

since by convention we have $T_{0}(\cdot)=1$ and so $t_{0}=1$. Eq. (4) allows one to express each $T_{1}\left(a^{k}\right)$ as a polynomial in $t_{1}, \ldots, t_{k}$ only, which we refer to as $p_{k}\left(t_{1}, \ldots, t_{k}\right)$. In particular, $p_{1}\left(t_{1}\right)=t_{1}, p_{2}\left(t_{1}, t_{2}\right)=$ $t_{1}^{2}-2 t_{2}, p_{3}\left(t_{1}, t_{2}, t_{3}\right)=3 t_{3}+t_{1}^{3}-3 t_{1} t_{2}$, and in general we have

$$
p_{k}\left(t_{1}, \ldots, t_{k}\right)=T_{1}\left(a^{k}\right)=(-1)^{k-1}\left(k t_{k}-\sum_{j=1}^{k-1}(-1)^{j-1} t_{k-j} p_{j}\left(t_{1}, \ldots, t_{j}\right)\right) .
$$

Since $1 \leq k \leq l<p$, each $t_{k}$ features in the condition on $T_{1}\left(a^{k}\right)$ in Eq. (5) and does so linearly. Therefore for each $\left(t_{1}^{\prime}, \ldots, t_{l}^{\prime}\right) \in\left(\mathbb{F}_{q}\right)^{l}$ there is a unique $\left(t_{1}, \ldots, t_{l}\right) \in\left(\mathbb{F}_{q}\right)^{l}$ such that for all $1 \leq k \leq l$ we have $t_{k}^{\prime}=p_{k}\left(t_{1}, \ldots, t_{k}\right)$, and vice versa. Eq. (2) thus becomes

$$
\#\left\{a \in \mathbb{F}_{q^{n}} \mid T_{1}(a)=t_{1}^{\prime}, T_{1}\left(a^{2}\right)=t_{2}^{\prime}, \ldots, T_{1}\left(a^{l}\right)=t_{l}^{\prime}\right\} .
$$

To evaluate Eq. (6) with what we call the direct method, let $\bar{n} \in\{1, \ldots, p-1\}$ and suppose $n \equiv \bar{n}(\bmod p)$. Then by introducing variables $a_{1}, \ldots, a_{l}$ and repeatedly applying Lemma 1, Eq. (6) equals

$$
\frac{1}{q^{l}} \#\left\{\left(a, a_{1}, \ldots, a_{l}\right) \in\left(\mathbb{F}_{q^{n}}\right)^{l+1} \mid a_{1}^{q}-a_{1}=a-t_{1}^{\prime} / \bar{n}, \ldots, a_{l}^{q}-a_{l}=a^{l}-t_{l}^{\prime} / \bar{n}\right\} .
$$

Note that if $t_{1}^{\prime}=\cdots=t_{l}^{\prime}=0$ then one need not introduce $\bar{n}$ at all, and the count (7) is valid for all $n \geq l$. Rather than work with this intersection one can alternatively define the curves $C_{k} / \mathbb{F}_{q}: a_{1}^{q}-a_{1}=a^{k}-t_{k}^{\prime} / \bar{n}$ for $1 \leq k \leq l$ and consider their fibre product, à la [52, §2]. In $\S 2.3$ we take a similar but more elementary approach, working only with an associated set of Artin-Schreier curves of much smaller genus, since there exists a practical algorithm for computing their zeta functions due to Lauder and Wan [35]. We do so by evaluating the count (6) indirectly, which allows one to solve the prescribed traces problem for all $q^{l}$ such $F_{q}\left(n, t_{1}, \ldots, t_{l}\right)$ simultaneously.

\subsection{A transform of the prescribed traces problem}

In this subsection we transform the problem of counting the number of elements of $\mathbb{F}_{q^{n}}$ with prescribed traces to the problem of counting the number of elements for which linear combinations of the trace functions evaluate to 1 . The transform is more general than is required for the target problem of interest and may be applied to any number of functions from $\mathbb{F}_{q^{n}}$ to $\mathbb{F}_{q}$. Therefore let $m \geq 1$ be the number of such functions. We first fix some notation. 
We require a bijection from the integers $\left\{0, \ldots, q^{m}-1\right\}$ to $\left(\mathbb{F}_{q}\right)^{m}$, the image of an input $i$ being denoted by $\mathbf{i}$. One can for instance take the base- $q$ expansion of $i$ to give $i_{m-1}^{\prime} q^{m-1}+\cdots+i_{0}^{\prime}$ and then set $\mathbf{i}=\left(i_{m-1}, \ldots, i_{0}\right)=\left(\tau\left(i_{m-1}^{\prime}\right), \ldots, \tau\left(i_{0}^{\prime}\right)\right)$, where $\tau:\{0, \ldots, q-1\} \rightarrow \mathbb{F}_{q}$ is defined by fixing a degree $r$ monic irreducible $f \in \mathbb{F}_{p}[x]$ and a polynomial basis for $\mathbb{F}_{p^{r}} / \mathbb{F}_{p}$, and mapping the base- $p$ expansion of an integer in $\{0, \ldots, q-1\}$ to the polynomial with those coefficients. Note that according to this definition, $\mathbf{0}=(0, \ldots, 0)$ is the all-zero vector in $\left(\mathbb{F}_{q}\right)^{m}$.

Let $f_{0}, \ldots, f_{m-1}: \mathbb{F}_{q^{n}} \rightarrow \mathbb{F}_{q}$ be any functions and let $\mathbf{f}=\left(f_{m-1}, \ldots, f_{0}\right)$. For $\mathbf{i}=\left(i_{m-1}, \ldots, i_{0}\right), \mathbf{j}=$ $\left(j_{m-1}, \ldots, j_{0}\right) \in\left(\mathbb{F}_{q}\right)^{m}$ let $\mathbf{i} \cdot \mathbf{j}$ denote the usual inner product. For any $\mathbf{i} \in\left(\mathbb{F}_{q}\right)^{m}$, let $\mathbf{i} \cdot \mathbf{f}$ denote the function

$$
\sum_{k=0}^{m-1} i_{k} f_{k}: \mathbb{F}_{q^{n}} \rightarrow \mathbb{F}_{q}
$$

and let $V_{1}(\mathbf{i} \cdot \mathbf{f})$ denote the number of elements of $\mathbb{F}_{q^{n}}$ for which $\mathbf{i} \cdot \mathbf{f}$ evaluates to 1 . We define $V_{1}(\mathbf{0} \cdot \mathbf{f})$ to be $q^{n}$. Finally, let $N(\mathbf{j})=N\left(j_{m-1}, \ldots, j_{0}\right)$ denote the number of $a \in \mathbb{F}_{q^{n}}$ such that $f_{k}(a)=j_{k}$, for $k=0, \ldots, m-1$.

Our goal is to express any $N(\mathbf{j})$ in terms of the $V_{1}(\mathbf{i} \cdot \mathbf{f})$, but we begin by first solving the inverse problem, i.e., expressing any $V_{1}(\mathbf{i} \cdot \mathbf{f})$ in terms of the $N(\mathbf{j})$.

Lemma 3. With the notation as above, for $\mathbf{i} \in\left(\mathbb{F}_{q}\right)^{m} \backslash\{\mathbf{0}\}$ we have

$$
V_{1}(\mathbf{i} \cdot \mathbf{f})=\sum_{\mathbf{i} \cdot \mathbf{j}=1} N(\mathbf{j})
$$

Proof. By definition, we have $V_{1}(\mathbf{i} \cdot \mathbf{f})=\#\left\{a \in \mathbb{F}_{q^{n}} \mid \mathbf{i} \cdot \mathbf{f}(a)=1\right\}=\#\left\{a \in \mathbb{F}_{q^{n}} \mid \sum_{k=0}^{m-1} i_{k} f_{k}(a)=1\right\}$. Since $N(\mathbf{j})$ counts precisely those $a \in \mathbb{F}_{q^{n}}$ such that $f_{k}(a)=j_{k}$, we must count over all those $\mathbf{j}$ for which $\sum_{k=0}^{m-1} i_{k} f_{k}(a)=1$, i.e., those such that $\sum_{k=0}^{m-1} i_{k} j_{k}=1$.

Writing Eq. (8) in matrix form, for $i, j \in\left\{0, \ldots, q^{m}-1\right\}$ we have

$$
\left[V_{1}(\mathbf{i} \cdot \mathbf{f})\right]^{T}=S_{q, m} \cdot[N(\mathbf{j})]^{T},
$$

where

$$
\left(S_{q, m}\right)_{i, j}=\left\{\begin{array}{ll}
1 & \text { if } \mathbf{i} \cdot \mathbf{j}=1 \text { or if } \mathbf{i}=\mathbf{0} \\
0 & \text { otherwise }
\end{array} .\right.
$$

We have the following lemma.

Lemma 4. For all prime powers $q=p^{r}$ and $m \geq 1$, the $q^{m} \times q^{m}$ matrix $S_{q, m}$ is invertible over $\mathbb{Q}$.

Proof. Indexing the rows and columns by $i$ and $j$ for $0 \leq i, j \leq q^{m}-1$, the 0 -th row of $S_{q, m}$ consists of 1 's only, while besides the initial 1 , the 0 -th column consists of 0's only. Therefore no $\mathbb{Q}$-linear combination of rows 1 to $q^{m}-1$ can cancel the 1 in position $(0,0)$. Hence if one shows that the submatrix

$$
S_{1 \leq i, j \leq q^{m}-1}= \begin{cases}1 & \text { if } \mathbf{i} \cdot \mathbf{j}=1 \\ 0 & \text { otherwise }\end{cases}
$$

of $S_{q, m}$ is invertible then we are done, since this implies that $S_{q, m}$ has full rank. We claim that the inverse of $S$ is

$$
S_{1 \leq i, j \leq q^{m}-1}^{\text {inv }}=\frac{1}{q^{m-1}}\left\{\begin{aligned}
1 & \text { if } \mathbf{i} \cdot \mathbf{j}=1 \\
-1 & \text { if } \mathbf{i} \cdot \mathbf{j}=0 \\
0 & \text { otherwise }
\end{aligned}\right.
$$


Let $R=S \cdot S^{\text {inv }}$. Then for $1 \leq i, j \leq q^{m}-1$ one has

$$
R_{i, j}=\sum_{k=1}^{q^{m}-1} S_{i, k} \cdot S_{k, j}^{\text {inv }}=\sum_{\substack{k=1, \mathbf{i} \cdot \mathbf{k}=1, \mathbf{j} \cdot \mathbf{k}=1}}^{q^{m}-1} 1-\sum_{\substack{k=1, \mathbf{i} \cdot \mathbf{k}=1, \mathbf{j} \cdot \mathbf{k}=0}}^{q^{m}-1} 1 .
$$

If $i=j$ then the second term of the r.h.s. of Eq. (9) is zero, while the first is $q^{m-1}$, since if one chooses a non-zero component $i_{l}$ of $\mathbf{i}$ (of which there is at least one as $i \neq 0$ ), then one can freely choose the $m-1$ coefficients of $\mathbf{k}$ other than $k_{l}$, while the condition $\mathbf{i} \cdot \mathbf{k}=1$ entails that

$$
k_{l}=\left(1-\sum_{\substack{w=0, w \neq l}}^{m-1} i_{w} k_{w}\right) / i_{l}
$$

which is well-defined because $i_{l}$ is invertible.

Now assume $i \neq j$. If possible choose $l, l^{\prime}$ such that $l \neq l^{\prime}$ and $i_{l} \neq 0$ and $j_{l^{\prime}} \neq 0$. Then considering the set of all $k$ for which $\mathbf{i} \cdot \mathbf{k}=1$ as described above, as $k_{l^{\prime}}$ varies over $\mathbb{F}_{q}$, so does $k_{l^{\prime}} j_{l^{\prime}}$. Hence both terms of the r.h.s. of Eq. (9) are precisely $q^{m-2}$, since there are $m-2$ free components of $\mathbf{k}$. For the remaining case where $\mathbf{i}$ and $\mathbf{j}$ both have only one non-zero component, in position $l$ say, then both terms of the r.h.s. of Eq. (9) are zero, since for the first there is no $k_{l}$ for which $i_{l} k_{l}=1$ and $j_{l} k_{l}=1$ since $i_{l} \neq j_{l}$, while for the second the condition $\mathbf{j} \cdot \mathbf{k}=0$ implies $k_{l}=0$, in which case $i_{l} k_{l}=1$ can not hold. Therefore, $R$ is the $q^{m}-1 \times q^{m}-1$ identity matrix.

To compute $N(\mathbf{0})$, we claim that

$$
N(\mathbf{0})=V_{1}(\mathbf{0} \cdot \mathbf{f})-\frac{1}{q^{m-1}} \sum_{i=1}^{q^{m}-1} V_{1}(\mathbf{i} \cdot \mathbf{f}) .
$$

Since $V_{1}(\mathbf{0} \cdot \mathbf{f})=q^{n}=\sum_{j=0}^{q^{m}-1} N(\mathbf{j})$ by definition, Eq. (10) is equivalent to

$$
\sum_{j=1}^{q^{m}-1} N(\mathbf{j})=\frac{1}{q^{m-1}} \sum_{i=1}^{q^{m}-1} V_{1}(\mathbf{i} \cdot \mathbf{f})=\frac{1}{q^{m-1}} \sum_{i=1}^{q^{m}-1} \sum_{\mathbf{i} \cdot \mathbf{j}=1} N(\mathbf{j}),
$$

with the latter equality given by Eq. (8). Considering the r.h.s. of Eq. (11), the number of occurrences of $N(\mathbf{j})$ is $q^{m-1}$ since as we argued previously if one fixes an $l$ for which $j_{l} \neq 0$, then one can choose the components of $\mathbf{i}$ other than $i_{l}$ freely, while $i_{l}$ is then fixed by the condition $\mathbf{i} \cdot \mathbf{j}=1$. We have therefore proven the following:

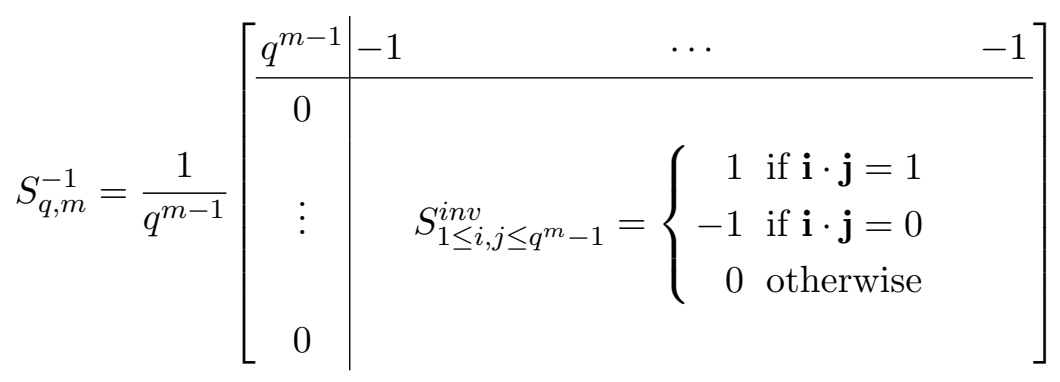

Thus in order to compute any of the $q^{m}$ possible outputs $N(\mathbf{j})$ of any set of $m$ functions $\mathbf{f}$, it is sufficient to count the number of evaluations to 1 of all the $q^{m}-1$ non-zero $\mathbb{F}_{q}$-linear combinations of the functions, and then apply $S_{q, m}^{-1}$. In particular, one may choose the $f_{0}, \ldots, f_{m-1}$ to be any subset of the trace functions $T_{1}, \ldots, T_{n}$, or in our case of interest, the trace functions $T_{1}, \ldots, T_{l}$. 


\subsection{Indirect method}

As per the transform of the previous subsection let $\mathbf{f}=\left(T_{1}\left(a^{l}\right), T_{1}\left(a^{l-1}\right), \ldots, T_{1}(a)\right)$ and for $1 \leq i \leq q^{l}-1$ let $\mathbf{i}=\left(i_{l-1}, \ldots, i_{0}\right)$. Computing formulae for $V_{1}(\mathbf{i} \cdot \mathbf{f})$ for each such $i$ produces formulae for all $q^{l}$ possible counts in Eq. (6), which then uniquely determine $F_{q}\left(n, t_{1}, \ldots, t_{l}\right)$. For $\bar{n} \in\{1, \ldots, p-1\}$ and all $n \equiv \bar{n}$ $(\bmod p)$, applying Lemma 1 we have

$$
\begin{aligned}
V_{1}(\mathbf{i} \cdot \mathbf{f}) & =\#\left\{a \in \mathbb{F}_{q^{n}} \mid \sum_{k=0}^{l-1} i_{k} f_{k}(a)=1\right\} \\
& \left.=\frac{1}{q} \#\left\{\left(a, a_{1}\right) \in\left(\mathbb{F}_{q^{n}}\right)^{2} \mid a_{1}^{q}-a_{1}=-\frac{1}{\bar{n}}+\sum_{k=0}^{l-1} i_{k} a^{k+1}\right)\right\} .
\end{aligned}
$$

In order to compute the zeta functions of the Artin-Schreier curves in (13) we use a $p$-adic point counting algorithm due to Lauder and Wan that is well suited to this purpose [35, Algorithm 25]. Let $f \in \mathbb{F}_{q}[a]$ be of degree $d$ not divisible by $p$, let $C_{f} / \mathbb{F}_{q}$ be the affine curve with equation $a_{1}^{p}-a_{1}=f(a)$ and let $\tilde{C}_{f}$ denote the unique smooth projective curve that is birational to $C_{f}$. By Weil [56], we know that the zeta function $Z\left(\tilde{C}_{f}, T\right)$ of $\tilde{C}_{f}$ satisfies

$$
Z\left(\tilde{C}_{f}, T\right)=\frac{P\left(\tilde{C}_{f}, T\right)}{(1-T)(1-q T)}
$$

where $P$ is polynomial of degree $2 g$ with $g=(p-1)(d-1) / 2$ the genus of $\tilde{C}_{f}$, and which factorises as $\prod_{k=1}^{2 g}\left(1-\omega_{k} t\right)$ with $\left|\omega_{k}\right|=\sqrt{q}$. Since $\tilde{C}_{f}$ is birational to $C_{f}$ their respective zeta functions differ only by a constant factor and thus to compute $Z\left(C_{f}, T\right)$ it suffices to compute $Z\left(\tilde{C}_{f}, T\right)$. An exponential sum approach also allows one to deduce the form of $Z\left(C_{f}, T\right)$ directly $[35, \S 2.2]$. We have the following.

Theorem 2. [35, Theorem 1] The zeta function of the smooth projective curve $\tilde{C}_{f}$ (and thus the affine curve $C_{f}$ ) may be computed deterministically in $\tilde{\mathcal{O}}\left(d^{5} p^{4} r^{3}\right)$ bit operations.

We note that one does not need to compute the unique smooth projective curve $\tilde{C}_{f}$ in order to apply the Lauder-Wan algorithm.

Let the right hand side of the curves in (13) be denoted by $\bar{p}_{\mathbf{i}, \bar{n}}(a)$ and denote each curve by

$$
C_{\mathbf{i}, \bar{n}} / \mathbb{F}_{q}: a_{1}^{q}-a_{1}=\bar{p}_{\mathbf{i}, \bar{n}}(a) .
$$

The degree of $\bar{p}_{\mathbf{i}, \bar{n}}(a)$ is $k^{\prime}+1$ where $k^{\prime}$ is the largest $0 \leq k^{\prime} \leq l-1$ such that $i_{k^{\prime}} \neq 0$. Since $k^{\prime}+1 \leq l<p$, for all $1 \leq i \leq q^{l}-1$ the degree of $\bar{p}_{\mathbf{i}, \bar{n}}(a)$ is less than $p$ and thus not divisible by $p$, and so this precondition of the Lauder-Wan algorithm is satisfied. However, the Lauder-Wan algorithm can only be applied when the left hand side of (15) is $a_{1}^{p}-a_{1}$; we therefore need to reduce to this case. This issue was already addressed in [3, Corollary 1] for the case $p=2$ (albeit with a sign error) using a theorem of Kani-Rosen [28] and by studying quotients arising from the action of automorphisms of the left hand side only. The correct generalisation is as follows, where for an affine curve $C / \mathbb{F}_{q}$ we denote by $\# C\left(\mathbb{F}_{q^{n}}\right)$ the number of $\mathbb{F}_{q^{n-}}$ rational affine points of $C$.

Lemma 5. Let $p \geq 2$ be prime, for $r \geq 1$ let $q=p^{r}$, let $f \in \mathbb{F}_{q}[x]$, let $C / \mathbb{F}_{q}$ be the Artin-Schreier curve $y^{q}-y=f(x)$ and for $\alpha \in \mathbb{F}_{q}^{\times}$let $C_{\alpha} / \mathbb{F}_{q}$ be the Artin-Schreier curve $y^{p}-y=\alpha f(x)$. Then for all $n \geq 1$ we have

$$
(p-1) \# C\left(\mathbb{F}_{q^{n}}\right)-\sum_{\alpha \in \mathbb{F}_{q}^{\times}} \# C_{\alpha}\left(\mathbb{F}_{q^{n}}\right)=(p-q) q^{n} .
$$

Thus computing the zeta function of $C / \mathbb{F}_{q}$ reduces to computing the zeta functions of the $q-1$ curves $C_{\alpha} / \mathbb{F}_{q}$. Lemma 5 may be proven using an exponential sum argument and such a proof can be found (in Persian) in [2]. For completeness we include it here and thank Omran Ahmadi for communicating to us its translation. 
Proof. For $m, r \geq 1$ positive integers let $\operatorname{Tr}_{m}: \mathbb{F}_{p^{m}} \rightarrow \mathbb{F}_{p}: x \mapsto x+x^{p}+x^{p^{2}}+\cdots+x^{p^{m-1}}$ denote the absolute trace function, and let $\operatorname{Tr}_{r m \mid r}: \mathbb{F}_{p^{r m}} \rightarrow \mathbb{F}_{p^{r}}: x \mapsto x+x^{p^{r}}+x^{p^{2 r}}+\cdots+x^{p^{(m-1) r}}$ denote the trace. By Lemma 1 (1) if $\operatorname{Tr}_{r n}(\alpha f(a))=0$, then there are $p$ points on $C_{\alpha}$ with $x$-coordinates equal to $a$, and otherwise none since $\operatorname{Tr}_{r n}\left(y^{p}-y\right)=0$ for all $y \in \mathbb{F}_{p^{r n}}$. Therefore the number of points on $C_{\alpha}$ with $x$-coordinate equal to $a$ is $\sum \psi\left(\operatorname{Tr}_{r n}(\alpha f(a))\right)$ where the sum is over the additive characters of $\mathbb{F}_{p}$. Denoting the set of all additive characters of $\mathbb{F}_{p}$ by $\Psi$ and its trivial character by $\psi_{0}$ we have

$$
\# C_{\alpha}\left(\mathbb{F}_{p^{r n}}\right)=\sum_{a \in \mathbb{F}_{p^{r n}}} \sum_{\psi \in \Psi} \psi\left(\operatorname{Tr}_{r n}(\alpha f(a))\right)=p^{r n}+\sum_{a \in \mathbb{F}_{p} r n} \sum_{\psi \neq \psi_{0}} \psi\left(\operatorname{Tr}_{r n}(\alpha f(a))\right)
$$

Summing over all $\alpha \in \mathbb{F}_{p^{r}}^{\times}$we obtain

$$
\begin{aligned}
S=\sum_{\alpha \in \mathbb{F}_{p^{r}}^{\times}} \# C_{\alpha}\left(\mathbb{F}_{p^{r n}}\right) & =p^{r n}\left(p^{r}-1\right)+\sum_{\alpha \in \mathbb{F}_{p^{r}}^{\times}} \sum_{a \in \mathbb{F}_{p^{r n}}} \sum_{\psi \neq \psi_{0}} \psi\left(\operatorname{Tr}_{r n}(\alpha f(a))\right) \\
& =p^{r n}\left(p^{r}-1\right)+\sum_{a \in \mathbb{F}_{p^{r n}}} \sum_{\alpha \in \mathbb{F}_{p^{r}}^{\times}} \sum_{\psi \neq \psi_{0}} \psi\left(\operatorname{Tr}_{r n}(\alpha f(a))\right) .
\end{aligned}
$$

Since $\alpha \in \mathbb{F}_{p^{r}}$, we have

$$
\operatorname{Tr}_{r n}(\alpha f(a))=\operatorname{Tr}_{r}\left(\operatorname{Tr}_{r n \mid r}(\alpha f(a))\right)=\operatorname{Tr}_{r}\left(\alpha \operatorname{Tr}_{r n \mid r}(f(a))\right)
$$

This implies that if $\operatorname{Tr}_{r n \mid r}(f(a))=0$ for an $a \in \mathbb{F}_{p^{r n}}$, then

$$
\sum_{\alpha \in \mathbb{F}_{p^{r}}^{\times}} \sum_{\psi \neq \psi_{0}} \psi\left(\operatorname{Tr}_{r n}(\alpha f(a))\right)=\sum_{\alpha \in \mathbb{F}_{p^{r}}^{\times}} \sum_{\psi \neq \psi_{0}} \psi\left(\operatorname{Tr}_{r}\left(\alpha \operatorname{Tr}_{r n \mid r}(f(a))\right)\right)=(p-1)\left(p^{r}-1\right),
$$

and otherwise

$$
\sum_{\alpha \in \mathbb{F}_{p^{r}}^{\times}} \sum_{\psi \neq \psi_{0}} \psi\left(\operatorname{Tr}_{r n}(\alpha f(a))\right)=\sum_{\alpha \in \mathbb{F}_{p^{r}}^{\times}} \sum_{\psi \neq \psi_{0}} \psi\left(\operatorname{Tr}_{r}\left(\alpha \operatorname{Tr}_{r n \mid r}(f(a))\right)\right)=1-p .
$$

If we let

$$
T=\#\left\{a \in \mid \operatorname{Tr}_{r n \mid r}(f(a))=0\right\}
$$

and

$$
U=\#\left\{a \in \mid \operatorname{Tr}_{r n \mid r}(f(a)) \neq 0\right\},
$$

then $T+U=p^{r n}$. Using this fact and combining Equations (17), (18) and (19), we obtain

$$
\begin{aligned}
S & =p^{r n}\left(p^{r}-1\right)+\left(p^{r}-1\right)(p-1) T+(1-p) U \\
& =p^{r n}\left(p^{r}-1\right)+\left(p^{r}-1\right)(p-1) T+(1-p)\left(p^{r n}-T\right) \\
& =(p-1) p^{r} T+p^{r n}\left(p^{r}-p\right)
\end{aligned}
$$

Applying Lemma 1 (1) again we have

$$
\# C\left(\mathbb{F}_{p^{r n}}\right)=p^{r} T
$$

and hence by Eq. (20) we have

$$
(p-1) \# C\left(\mathbb{F}_{p^{r n}}\right)-\sum_{\alpha \in \mathbb{F}_{p^{r}}^{\times}} \# C_{\alpha}\left(\mathbb{F}_{p^{r n}}\right)=\left(p-p^{r}\right) p^{r n}
$$


Proof of Theorem 1: Fix $\bar{n} \in\{1, \ldots, p-1\}$. By transform (12), in order to compute all $F_{q}\left(n, t_{1}, \ldots, t_{l}\right)$ one need only compute $V_{1}(\mathbf{i} \cdot \mathbf{f})$ for all $1 \leq i \leq q^{l}-1$. For each such $i$ let $d(\mathbf{i})$ denote the degree of $\bar{p}_{\mathbf{i}, \bar{n}}(a)$, which we recall is $\left(k^{\prime}+1\right)$ where $k^{\prime}$ is the largest $0 \leq k^{\prime} \leq l-1$ such that $i_{k^{\prime}} \neq 0$. The number of $i$ 's for which $d(\mathbf{i})$ is $l, l-1, \ldots, 1$ is therefore $(q-1) q^{l-1},(q-1) q^{l-2}, \ldots, q-1$, respectively.

We first count the number of roots - counted with multiplicities, since they may not all be distinct which appear in the numerator of the zeta function of the curve $C_{\mathbf{i}, \bar{n}} / \mathbb{F}_{q}$ featured in $V_{1}(\mathbf{i} \cdot \mathbf{f})$. For $q=p$ the genus of $C_{\mathbf{i}, \bar{n}} / \mathbb{F}_{p}$ is $(p-1)(d(\mathbf{i})-1) / 2$ and so there are $(p-1)(d(\mathbf{i})-1)$ roots. The total number of roots over all $1 \leq i \leq p^{l}-1$ is therefore

$$
(p-1) \sum_{i=1}^{p^{l}-1}(d(\mathbf{i})-1)=(p-1) \sum_{d=1}^{l}(p-1) p^{d-1}(d-1)=(p-1)^{2} \sum_{d=1}^{l-1} d p^{d}=p^{l}(p l-p-l)+p .
$$

For $q=p^{r}$ with $r>1$, as per Lemma 5 we adapt the curves in Eq. (15), so for $\alpha \in \mathbb{F}_{q}^{\times}$let

$$
C_{\alpha, \mathbf{i}, \bar{n}} / \mathbb{F}_{q}: a_{1}^{p}-a_{1}=\alpha \bar{p}_{\mathbf{i}, \bar{n}}(a) .
$$

Since the number of roots of $C_{\mathbf{i}, \bar{n}} / \mathbb{F}_{q}$ is the sum over $\alpha \in \mathbb{F}_{q}^{\times}$of the number of roots of $C_{\alpha, \mathbf{i}, \bar{n}} / \mathbb{F}_{q}$, the total number of roots is therefore

$$
\begin{aligned}
(q-1)(p-1) \sum_{i=1}^{q^{l}-1}(d(\mathbf{i})-1) & =(q-1)(p-1) \sum_{d=1}^{l}(q-1) q^{d-1}(d-1)=(q-1)^{2}(p-1) \sum_{d=1}^{l-1} d q^{d} \\
& =(p-1)\left(q^{l}(q l-q-l)+q\right) .
\end{aligned}
$$

Thus $N$ is as stated in the theorem and the (not necessarily distinct) roots $\omega_{1}, \ldots, \omega_{N} \in \overline{\mathbb{Z}}$ all have norm $\sqrt{q}$. The $v_{1}, \ldots, v_{N}$ corresponding to each $\mathbf{t}=\left(t_{1}, \ldots, t_{l}\right)$ follow immediately from transform (12). In particular, for $q=p$ we have

$$
F_{p}(n, \mathbf{0})=\frac{1}{p^{l-1}}\left(p^{n+l-1}-\sum_{i=1}^{p^{l}-1} V_{1}(\mathbf{i} \cdot \mathbf{f})\right)=p^{n}-\frac{1}{p^{l}} \sum_{i=1}^{p^{l}-1}\left(p^{n}-\sum_{k=1}^{(p-1)(d(\mathbf{i})-1)} \omega_{i, k}^{n}\right)=\frac{1}{p^{l}}\left(p^{n}+\sum_{k=1}^{N} \omega_{k}^{n}\right),
$$

while for $\mathbf{t} \neq \mathbf{0}$ we have

$$
F_{p}(n, \mathbf{t})=\frac{1}{p^{l-1}}\left(\sum_{\mathbf{i} \cdot \mathbf{t}=1} V_{1}(\mathbf{i} \cdot \mathbf{f})-\sum_{\mathbf{i} \cdot \mathbf{t}=0, \mathbf{i} \neq \mathbf{0}} V_{1}(\mathbf{i} \cdot \mathbf{f})\right)=\frac{1}{p^{l}}\left(p^{n}+\sum_{k=1}^{N} v_{k} \omega_{k}^{n}\right),
$$

where the $v_{k}$ are in $\{0, \pm 1\}$ and the $p^{n}$ arises as there are $p^{l-1}$ indices $i$ for which $\mathbf{i} \cdot \mathbf{t}=1$, while there are $p^{l-1}-1$ non-zero indices $i$ for which $\mathbf{i} \cdot \mathbf{t}=0$. For $q=p^{r}$ with $r>1$, we have

$$
\# C_{\alpha, \mathbf{i}, \bar{n}}\left(\mathbb{F}_{q^{n}}\right)=q^{n}-\sum_{k=1}^{(p-1)(d(\mathbf{i})-1)} \omega_{\alpha, i, k}^{n}
$$

and hence

$$
\sum_{\alpha \in \mathbb{F}_{q}^{\times}} \# C_{\alpha, \mathbf{i}, \bar{n}}\left(\mathbb{F}_{q^{n}}\right)=(q-1) q^{n}-\sum_{k=1}^{(q-1)(p-1)(d(\mathbf{i})-1)} \omega_{i, k}^{n},
$$

and by Lemma 5 we have

$$
\begin{aligned}
\# C_{\mathbf{i}, \bar{n}}\left(\mathbb{F}_{q^{n}}\right) & =\frac{p-q}{p-1} q^{n}+\frac{q-1}{p-1}\left(q^{n}-\sum_{k=1}^{(q-1)(p-1)(d(\mathbf{i})-1)} \omega_{i, k}^{n}\right) \\
& =q^{n}-\frac{q-1}{p-1} \sum_{k=1}^{(q-1)(p-1)(d(\mathbf{i})-1)} \omega_{i, k}^{n},
\end{aligned}
$$


and

$$
V_{1}(\mathbf{i} \cdot \mathbf{f})=\frac{1}{q}\left(q^{n}-\frac{q-1}{p-1} \sum_{k=1}^{(q-1)(p-1)(d(\mathbf{i})-1)} \omega_{i, k}^{n}\right) .
$$

As before, by (12) we have

$$
\begin{aligned}
F_{q}(n, \mathbf{0})=\frac{1}{q^{l-1}}\left(q^{n+l-1}-\sum_{i=1}^{q^{l}-1} V_{1}(\mathbf{i} \cdot \mathbf{f})\right) & =q^{n}-\frac{1}{q^{l}} \sum_{i=1}^{q^{l}-1}\left(q^{n}-\frac{q-1}{p-1} \sum_{k=1}^{(q-1)(p-1)(d(\mathbf{i})-1)} \omega_{i, k}^{n}\right) \\
& =\frac{1}{q^{l}}\left(q^{n}+\frac{q-1}{p-1} \sum_{k=1}^{N} \omega_{k}^{n}\right),
\end{aligned}
$$

while for $\mathbf{t} \neq \mathbf{0}$ we have

$$
F_{q}(n, \mathbf{t})=\frac{1}{q^{l-1}}\left(\sum_{\mathbf{i} \cdot \mathbf{t}=1} V_{1}(\mathbf{i} \cdot \mathbf{f})-\sum_{\mathbf{i} \cdot \mathbf{t}=0, \mathbf{i} \neq \mathbf{0}} V_{1}(\mathbf{i} \cdot \mathbf{f})\right)=\frac{1}{q^{l}}\left(q^{n}+\frac{q-1}{p-1} \sum_{k=1}^{N} v_{k} \omega_{k}^{n}\right) .
$$

In all cases, for fixed $p$ and $q$, as $n \rightarrow \infty$ with $n \equiv \bar{n}(\bmod p)$ we have

$$
F_{q}\left(n, t_{1}, \ldots, t_{l}\right)=q^{n-l}+\mathcal{O}\left(q^{n / 2}\right) .
$$

Regarding the complexity claim, for $q=p$, by Theorem 2 the cost of computing all the relevant zeta functions in terms of the number of bit operations is

$$
\sum_{i=1}^{p^{l}-1} \tilde{\mathcal{O}}\left(d(\mathbf{i})^{5} p^{4}\right)=\sum_{j=1}^{l}(p-1) p^{j-1} \cdot \tilde{\mathcal{O}}\left(j^{5} p^{4}\right)=p^{l} \cdot \tilde{\mathcal{O}}\left(l^{5} p^{4}\right) .
$$

For $q=p^{r}$ with $r>1$ we employ Lemma 5 and similarly obtain a cost in terms of the number of bit operations of

$$
(q-1) \sum_{i=1}^{q^{l}-1} \tilde{\mathcal{O}}\left(d(\mathbf{i})^{5} p^{4} r^{3}\right)=(q-1) \sum_{j=1}^{l}(q-1) q^{j-1} \cdot \tilde{\mathcal{O}}\left(j^{5} p^{4} r^{3}\right)=q^{l+1} \cdot \tilde{\mathcal{O}}\left(l^{5} p^{4} r^{3}\right)
$$

2.3.1 Some remarks on the algorithm. Firstly, note that the 'cost per $F_{q}\left(n, t_{1}, \ldots, t_{l}\right)$ ' is just $\tilde{\mathcal{O}}\left(l^{5} p^{4}\right)$ and $q \cdot \tilde{\mathcal{O}}\left(l^{5} p^{4} r^{3}\right)$ bit operations for $r=1$ and $r>1$, respectively. The algorithm is thus very efficient at computing all such $F_{q}\left(n, t_{1}, \ldots, t_{l}\right)$. Regarding practical efficiency, it should be possible to re-use much of the computation in the $q^{l}-1$ runs of the Lauder-Wan algorithm. It may also be possible for $r>1$ to work with the curve (15) rather than the curves (21), thus bypassing Lemma 5 and possibly reducing the complexity by a factor of $q-1$ in this case. Furthermore, if only one $F_{q}\left(n, t_{1}, \ldots, t_{l}\right)$ is required then there may be a way to exploit the direct method which is more efficient than computing either $2 q^{l-1}-1$ or $q^{l}-1$ zeta functions, as is required by the indirect method.

Secondly, as already noted there may be many repeats amongst the $\omega_{k}$ 's and so the number of summands in (1) may be far less than $N$, even for $F_{q}(n, \mathbf{0})$ to which they all contribute. Indeed, using the direct approach leads to formulae with less redundancy than the indirect method. For example, for $q=5$ and $l=4$, for the direct method the curve (7) is absolutely irreducible and has genus 860 , producing 1720 roots (not necessarily distinct), whereas the indirect method produces 6880 roots for $F_{q}(n, \mathbf{0})$ (not necessarily distinct) which is a factor of $p-1$ more. The advantage of the indirect method is that the genus of the arising curves is far smaller (0,2,4 and 6 in this example), making the computation of their zeta functions extremely efficient. One may be able to eliminate the redundancy: we provide an example of this in $\S 5$.

Finally, note that in contrast to the direct method, for the indirect method even if $\mathbf{t}=\mathbf{0}$, one needs to use $1 / \bar{n}$ as it features in (13), so the obtained formulae are only valid for $n$ coprime to $p$. 


\subsection{Reducing the prescribed coefficients problem to the prescribed traces problem}

We now show how to express our titular problem in terms of the prescribed traces problem. For the present case of interest for which $l<p$, the transform follows almost immediately from the work of Miers and Ruskey [40]; only a small change in the relevant definitions is needed.

For $a \in \mathbb{F}_{q^{n}}$ denote by $\bar{a}$ the string $\left(a, a^{q}, a^{q^{2}}, \ldots, a^{q^{n-1}}\right)$. Such a string is called periodic if there is a substring whose repeated concatenation gives $\bar{a}$; otherwise the string is called aperiodic. Observe that $\bar{a}$ is aperiodic if and only if $a$ does not belong to any proper subfield of $\mathbb{F}_{q^{n}}$. Let $A_{q}\left(n, t_{1}, \ldots, t_{l}\right)$ denote the number of aperiodic strings corresponding to elements of $\mathbb{F}_{q^{n}}$ in the above manner for which $T_{1}(a)=t_{1}, \ldots, T_{l}(a)=t_{l}$. If $\bar{a}$ is counted by $A_{q}\left(n, t_{1}, \ldots, t_{l}\right)$ then all rotations of $\bar{a}$ are distinct and produce the same trace values. By this and the above observation we thus have

$$
I_{q}\left(n, t_{1}, \ldots, t_{l}\right)=\frac{1}{n} A_{q}\left(n, t_{1}, \ldots, t_{l}\right) .
$$

Now let $f$ denote the minimum polynomial of $a$ over $\mathbb{F}_{q}$, which has degree $n / d$ for some $d \mid n$. Note that $T_{k}(a)$ is the coefficient of $x^{n-k}$ in $f^{d}$ [8, Lemma 2], so abusing notation slightly we also write $T_{k}(a)$ as $T_{k}\left(f^{d}\right)$. The multinomial theorem (cf. [40, Lemma 2.1]) gives the following.

Lemma 6. For all $k \geq 1$ and $d \geq 1$ we have

$$
T_{k}\left(f^{d}\right)=\sum_{\nu_{1}+2 \nu_{2}+\cdots+k \nu_{k}=k}\left(\begin{array}{c}
d \\
\nu_{1}, \ldots, \nu_{k}, d-\left(\nu_{1}+\cdots+\nu_{k}\right)
\end{array}\right) T_{1}(f)^{\nu_{1}} T_{2}(f)^{\nu_{2}} \cdots T_{k}(f)^{\nu_{k}} .
$$

This motivates the following definition. For a positive integer $d$ and $\mathbf{t}=\left(t_{1}, \ldots, t_{l}\right) \in\left(\mathbb{F}_{q}\right)^{l}$ define the map $\theta_{d}:\left(\mathbb{F}_{q}\right)^{l} \rightarrow\left(\mathbb{F}_{q}\right)^{l}: \mathbf{t} \rightarrow \mathbf{u}=\left(u_{1}, \ldots, u_{l}\right)$ by

$$
\begin{aligned}
u_{k} & =\sum_{\nu_{1}+2 \nu_{2}+\cdots+k \nu_{k}=k}\left(\begin{array}{c}
d \\
\nu_{1}, \ldots, \nu_{k}, d-\left(\nu_{1}+\cdots+\nu_{k}\right)
\end{array}\right) t_{1}^{\nu_{1}} t_{2}^{\nu_{2}} \cdots t_{k}^{\nu_{k}} \\
& =\sum_{\nu_{1}+2 \nu_{2}+\cdots+k \nu_{k}=k} d^{\left(\nu_{1}+\nu_{2}+\cdots+\nu_{k}\right)} \frac{t_{1}^{\nu_{1}}}{\nu_{1} !} \frac{t_{2}^{\nu_{2}}}{\nu_{2} !} \cdots \frac{t_{k}^{\nu_{k}}}{\nu_{k} !},
\end{aligned}
$$

where $d^{(m)}=d(d-1) \cdots(d-m+1)$ is the falling factorial.

For a propostion $P$ let $[P]$ denote its truth value. Since every periodic string is the repeated concatenation of an aperiodic string, we have (cf. [40, Eq. (2.5)]):

$$
F_{q}(n, \mathbf{u})=\sum_{d \mid n} \sum_{\mathbf{t} \in\left(\mathbb{F}_{q}\right)^{l}}\left[\theta_{d}(\mathbf{t})=\mathbf{u}\right] A_{q}\left(\frac{n}{d}, \mathbf{t}\right) .
$$

For the present case of interest there is precisely one $\mathbf{t}$ for each $\mathbf{u}$. Indeed, we have the following theorem - in which $\mu$ denotes the usual Möbius function - whose proof is identical to that given by Miers and Ruskey [40, Theorem 6.1].

Theorem 3. Let $q=p^{r}$ with $p$ an odd prime and let $\mathbf{t}=\left(t_{1}, \ldots, t_{l}\right)$ with $l<p$. Then

$$
I_{q}(n, \mathbf{t})= \begin{cases}\frac{1}{n} \sum_{\substack{d \mid n \\ p \nmid d}} \mu(d)\left(F_{q}\left(\frac{n}{d}, \mathbf{0}\right)-[p d \mid n] q^{n /(p d)}\right) & \text { if } \mathbf{t}=\mathbf{0}, \\ \frac{1}{n} \sum_{\substack{d \mid n \\ p \nmid d}} \mu(d) F_{q}\left(\frac{n}{d}, \theta_{d^{-1}}(\mathbf{t})\right) & \text { otherwise. }\end{cases}
$$


First note that the inverse of $d$ is computed $\bmod p$. Also note that since our algorithm does not address the $n \equiv 0(\bmod p)$ cases, the condition $p \nmid d$ in the summations is vacuous and the second term in each summand of the $\mathbf{t}=\mathbf{0}$ case is always zero. Therefore, for all $\mathbf{t}$ we have

$$
I_{q}(n, \mathbf{t})=\frac{1}{n} \sum_{d \mid n} \mu(d) F_{q}\left(\frac{n}{d}, \theta_{d^{-1}}(\mathbf{t})\right)=\frac{1}{n}\left(q^{n-l}+\mathcal{O}\left(q^{n / 2}\right)\right) .
$$

Finally, we note that for $q$ even only the first trace is prescribed, and this case was solved by Carlitz [7].

\subsection{Example: $F_{5}\left(n, t_{1}, t_{2}, t_{3}, t_{4}\right)$}

As a proof-of-concept example, for each $\bar{n} \in\{1,2,3,4\}$ we computed the zeta functions of all $5^{4}-1$ curves (15) that are required by the indirect method in order to compute each $F_{5}\left(n, t_{1}, t_{2}, t_{3}, t_{4}\right)$ for all $n \equiv \bar{n}(\bmod 5)$ with $n \geq 4$. The computations for each $\bar{n}$ took under a minute using Magma V22.2-3 on a $2.0 \mathrm{GHz}$ AMD Opteron computer. Note that Magma uses brute-force point counting over successive extension fields in order to compute the zeta functions. For this small example in which the genera are 0,2, 4 or 6 this is clearly not a concern. For much larger $p$ the Lauder-Wan algorithm should be used.

For the sake of space, in the following theorem we only give the formula for $F_{5}(n, 0,0,0,0)$, which turns out to be the same for each $\bar{n} \in\{1,2,3,4\}$. As per the transform (12) this count combines all $5^{4}-1$ zeta functions and thus has the greatest weight amongst all of the $F_{5}\left(n, t_{1}, t_{2}, t_{3}, t_{4}\right)$ formulae. For a polynomial $\gamma(X) \in \mathbb{Z}[X]$ let $\rho_{n}(\gamma)$ denote the sum of the $n$-th powers of the (complex) roots of $\gamma(X)$.

Theorem 4. For $n \geq 4$ and $(n, 5)=1$ we have

$$
\begin{aligned}
F_{5}(n, 0,0,0,0) & =5^{n-4}-\frac{1}{5^{4}}\left(160 \rho_{n}\left(\gamma_{2,1}\right)+16 \rho_{n}\left(\gamma_{2,2}\right)+164 \rho_{n}\left(\gamma_{4,1}\right)+16 \rho_{n}\left(\gamma_{4,2}\right)+116 \rho_{n}\left(\gamma_{4,3}\right)+25 \rho_{n}\left(\gamma_{8,1}\right)\right. \\
& +20 \rho_{n}\left(\gamma_{8,2}\right)+16 \rho_{n}\left(\gamma_{8,3}\right)+18 \rho_{n}\left(\gamma_{8,4}\right)+20 \rho_{n}\left(\gamma_{8,5}\right)+20 \rho_{n}\left(\gamma_{8,6}\right)+20 \rho_{n}\left(\gamma_{8,7}\right)+16 \rho_{n}\left(\gamma_{8,8}\right) \\
& +24 \rho_{n}\left(\gamma_{8,9}\right)+16 \rho_{n}\left(\gamma_{8,10}\right)+21 \rho_{n}\left(\gamma_{8,11}\right)+17 \rho_{n}\left(\gamma_{8,12}\right)+16 \rho_{n}\left(\gamma_{8,13}\right)+16 \rho_{n}\left(\gamma_{8,14}\right) \\
& +12 \rho_{n}\left(\gamma_{8,15}\right)+10 \rho_{n}\left(\gamma_{8,16}\right)+8 \rho_{n}\left(\gamma_{8,17}\right)+13 \rho_{n}\left(\gamma_{8,18}\right)+20 \rho_{n}\left(\gamma_{12,1}\right)+20 \rho_{n}\left(\gamma_{12,2}\right) \\
& +20 \rho_{n}\left(\gamma_{12,3}\right)+16 \rho_{n}\left(\gamma_{12,4}\right)+16 \rho_{n}\left(\gamma_{12,5}\right)+16 \rho_{n}\left(\gamma_{12,6}\right)+16 \rho_{n}\left(\gamma_{12,7}\right)+16 \rho_{n}\left(\gamma_{12,8}\right) \\
& +16 \rho_{n}\left(\gamma_{12,9}\right)+16 \rho_{n}\left(\gamma_{12,10}\right)+16 \rho_{n}\left(\gamma_{12,11}\right)+16 \rho_{n}\left(\gamma_{12,12}\right)+12 \rho_{n}\left(\gamma_{12,13}\right)+12 \rho_{n}\left(\gamma_{12,14}\right) \\
& \left.+12 \rho_{n}\left(\gamma_{12,15}\right)\right)
\end{aligned}
$$

where

$$
\begin{aligned}
\gamma_{2,1} & =X^{2}-5 \\
\gamma_{2,2} & =X^{2}+5 \\
\gamma_{4,1} & =X^{4}-5 X^{3}+15 X^{2}-25 X+25, \\
\gamma_{4,2} & =X^{4}+5 X^{2}+25 \\
\gamma_{4,3} & =X^{4}+5 X^{3}+15 X^{2}+25 X+25 \\
\gamma_{8,1} & =X^{8}-10 X^{7}+45 X^{6}-130 X^{5}+305 X^{4}-650 X^{3}+1125 X^{2}-1250 X+625, \\
\gamma_{8,2} & =X^{8}-5 X^{7}+10 X^{6}-25 X^{5}+75 X^{4}-125 X^{3}+250 X^{2}-625 X+625, \\
\gamma_{8,3} & =X^{8}-5 X^{7}+10 X^{6}+5 X^{5}-45 X^{4}+25 X^{3}+250 X^{2}-625 X+625, \\
\gamma_{8,4} & =X^{8}-5 X^{7}+15 X^{6}-35 X^{5}+80 X^{4}-175 X^{3}+375 X^{2}-625 X+625, \\
\gamma_{8,5} & =X^{8}-5 X^{7}+20 X^{6}-65 X^{5}+155 X^{4}-325 X^{3}+500 X^{2}-625 X+625, \\
\gamma_{8,6} & =X^{8}-15 X^{6}+105 X^{4}-375 X^{2}+625 \\
\gamma_{8,7} & =X^{8}-10 X^{6}+55 X^{4}-250 X^{2}+625, \\
\gamma_{8,8} & =X^{8}-5 X^{6}+25 X^{4}-125 X^{2}+625, \\
\gamma_{8,9} & =X^{8}+30 X^{4}+625 \\
\gamma_{8,10} & =X^{8}+5 X^{6}-20 X^{5}+5 X^{4}-100 X^{3}+125 X^{2}+625,
\end{aligned}
$$




$$
\begin{aligned}
& \gamma_{8,11}=X^{8}+5 X^{6}-10 X^{5}+5 X^{4}-50 X^{3}+125 X^{2}+625, \\
& \gamma_{8,12}=X^{8}+5 X^{6}+10 X^{5}+5 X^{4}+50 X^{3}+125 X^{2}+625, \\
& \gamma_{8,13}=X^{8}+5 X^{6}+20 X^{5}+5 X^{4}+100 X^{3}+125 X^{2}+625 \text {, } \\
& \gamma_{8,14}=X^{8}+5 X^{7}+10 X^{6}-5 X^{5}-45 X^{4}-25 X^{3}+250 X^{2}+625 X+625 \text {, } \\
& \gamma_{8,15}=X^{8}+5 X^{7}+10 X^{6}+25 X^{5}+75 X^{4}+125 X^{3}+250 X^{2}+625 X+625 \text {, } \\
& \gamma_{8,16}=X^{8}+5 X^{7}+15 X^{6}+35 X^{5}+80 X^{4}+175 X^{3}+375 X^{2}+625 X+625 \text {, } \\
& \gamma_{8,17}=X^{8}+5 X^{7}+20 X^{6}+65 X^{5}+155 X^{4}+325 X^{3}+500 X^{2}+625 X+625 \text {, } \\
& \gamma_{8,18}=X^{8}+10 X^{7}+45 X^{6}+130 X^{5}+305 X^{4}+650 X^{3}+1125 X^{2}+1250 X+625, \\
& \gamma_{12,1}=X^{12}-5 X^{11}+5 X^{10}+5 X^{9}+5 X^{8}+75 X^{7}-425 X^{6}+375 X^{5}+125 X^{4}+625 X^{3}+3125 X^{2}-15625 X+15625 \text {, } \\
& \gamma_{12,2}=X^{12}-5 X^{11}+10 X^{10}+5 X^{9}-20 X^{8}-125 X^{7}+575 X^{6}-625 X^{5}-500 X^{4}+625 X^{3}+6250 X^{2}-15625 X+15625 \text {, } \\
& \gamma_{12,3}=X^{12}-5 X^{11}+15 X^{10}-45 X^{9}+80 X^{8}-125 X^{7}+325 X^{6}-625 X^{5}+2000 X^{4}-5625 X^{3}+9375 X^{2}-15625 X+15625 \text {, } \\
& \gamma_{12,4}=X^{12}-10 X^{10}-5 X^{9}+80 X^{8}-425 X^{6}+2000 X^{4}-625 X^{3}-6250 X^{2}+15625 \text {, } \\
& \gamma_{12,5}=X^{12}-10 X^{10}+10 X^{9}+55 X^{8}-25 X^{7}-175 X^{6}-125 X^{5}+1375 X^{4}+1250 X^{3}-6250 X^{2}+15625 \text {, } \\
& \gamma_{12,6}=X^{12}-5 X^{10}-15 X^{9}+5 X^{8}+50 X^{7}+75 X^{6}+250 X^{5}+125 X^{4}-1875 X^{3}-3125 X^{2}+15625 \text {, } \\
& \gamma_{12,7}=X^{12}-5 X^{10}-10 X^{9}-20 X^{8}+25 X^{7}+325 X^{6}+125 X^{5}-500 X^{4}-1250 X^{3}-3125 X^{2}+15625 \text {, } \\
& \gamma_{12,8}=X^{12}-15 X^{9}+30 X^{8}-50 X^{7}+75 X^{6}-250 X^{5}+750 X^{4}-1875 X^{3}+15625 \\
& \gamma_{12,9}=X^{12}+15 X^{9}-20 X^{8}-50 X^{7}+75 X^{6}-250 X^{5}-500 X^{4}+1875 X^{3}+15625, \\
& \gamma_{12,10}=X^{12}+5 X^{10}-5 X^{9}+5 X^{8}+50 X^{7}+75 X^{6}+250 X^{5}+125 X^{4}-625 X^{3}+3125 X^{2}+15625 \text {, } \\
& \gamma_{12,11}=X^{12}+10 X^{10}+20 X^{9}+55 X^{8}+175 X^{7}+325 X^{6}+875 X^{5}+1375 X^{4}+2500 X^{3}+6250 X^{2}+15625 \text {, } \\
& \gamma_{12,12}=X^{12}+15 X^{10}-10 X^{9}+130 X^{8}-75 X^{7}+825 X^{6}-375 X^{5}+3250 X^{4}-1250 X^{3}+9375 X^{2}+15625 \text {, } \\
& \gamma_{12,13}=X^{12}+5 X^{11}+10 X^{10}+25 X^{9}+80 X^{8}+225 X^{7}+575 X^{6}+1125 X^{5}+2000 X^{4}+3125 X^{3}+6250 X^{2}+15625 X \\
& +15625 \\
& \gamma_{12,14}=X^{12}+5 X^{11}+15 X^{10}+25 X^{9}+5 X^{8}-125 X^{7}-425 X^{6}-625 X^{5}+125 X^{4}+3125 X^{3}+9375 X^{2}+15625 X+15625 \text {, } \\
& \gamma_{12,15}=X^{12}+5 X^{11}+20 X^{10}+75 X^{9}+230 X^{8}+600 X^{7}+1450 X^{6}+3000 X^{5}+5750 X^{4}+9375 X^{3}+12500 X^{2}+15625 X \\
& +15625 \text {. }
\end{aligned}
$$

Each of the above polynomials is the characteristic polynomial of the Frobenius endomorphism of an abelian variety. Regarding the potential periodicity in $n$ of the formula in Theorem 4 , whether the abelian varieties are supersingular or not can be determined by the following theorem of Stichtenoth and Xing [47, Prop. 1].

Theorem 5. Let $A$ be an abelian variety of dimension $g$ over $\mathbb{F}_{q}=\mathbb{F}_{p^{r}}$ and let $P(X)=X^{2 g}+a_{1} X^{2 g-1}+$ $\cdots+a_{g} X^{g}+\cdots+q^{g}$ be the characteristic polynomial of the Frobenius endomorphism on $A$. Then $A$ is supersingular if and only if for all $1 \leq k \leq$ one has $p^{\lceil k n / 2\rceil} \mid a_{k}$.

Applying Theorem 5 we see that $\gamma_{2,1}, \gamma_{2,2}, \gamma_{4,1}, \gamma_{4,2}, \gamma_{4,3}, \gamma_{8,2}, \gamma_{8,8}$ and $\gamma_{8,15}$ are the only supersingular cases. It is possible in principle to apply Kronecker's theorem to the phases of the non-supersingular Weil numbers to determine whether or not the formula in Theorem 4 is periodic in $n$. Note that the product of the powers of these polynomials as per Theorem 4 produces (a redundant version of) the characteristic polynomial of Frobenius of the intersection curve of genus 860 given by the direct method. Although it is not true for this polynomial that $a_{12} \equiv 0\left(\bmod 5^{6}\right)$, as would be required by Theorem 5 for supersingularity, it is possible (although seemingly unlikely) that the formula for the non-redundant method is periodic in $n$, which would require that all of the non-supersingular contributions cancel.

\section{An Algorithm for the Prescribed Traces Problem for $q=2$}

When $l \geq p$ the approach of $\S 2$ breaks due to the failure of Newton's identities in positive characteristic. For example, for $l=p$ we have $T_{1}\left(a^{p}\right)=t_{1}^{p}$ which does not feature $t_{p}$ and thus the system of linear trace conditions (6) is underdetermined. In this section we present an algorithm that obviates this issue 
and solves the prescribed traces problem for binary base fields for $l \leq 7$ and $n$ odd, which is potentially extendable to larger $l$. We focus here on the $q=2$ case, noting that the general $q=2^{r}$ case follows mutatis mutandis.

In contrast to the main algorithm we now permit any subset of the first $l$ traces to be prescribed: for subscripts $1 \leq l_{0}<\cdots<l_{m-1} \leq l, n \geq l_{m-1}$ and $t_{l_{0}}, \ldots, t_{l_{m-1}} \in \mathbb{F}_{2}$ we denote by $F_{2}\left(n, t_{l_{0}}, \ldots, t_{l_{m-1}}\right)$ the number of elements $a \in \mathbb{F}_{2^{n}}$ for which $T_{l_{0}}(a)=t_{l_{0}}, \ldots, T_{l_{m-1}}(a)=t_{l_{m-1}}$.

After presenting a specialised version of the transform from $\S 2.2$ in $\S 3.1$, we present the indirect method, in $\S 3.2$, as it is slightly simpler than the direct method, which we present in $\S 3.3$. The algebraic property upon which both methods rely is presented in $\S 3.4$. The values $T_{l}(1)=\left(\begin{array}{l}n \\ l\end{array}\right)$ feature repeatedly in the ensuing methods. We therefore begin with two preliminary results, the first of which is an immediate corollary of a theorem of Zabek [58].

Lemma 7. For $p$ a prime and $j \geq 1$ the period of the sequence $\left(\left(\begin{array}{l}0 \\ j\end{array}\right),\left(\begin{array}{l}1 \\ j\end{array}\right),\left(\begin{array}{l}2 \\ j\end{array}\right), \ldots\right) \bmod p$ is $p^{1+\left\lfloor\log _{p} j\right\rfloor}$.

We shall use the following corollary repeatedly.

Corollary 1. For $p$ a prime and $j \geq 1$ the period in $n$ of the set of vectors $\left(\left(\begin{array}{c}n \\ j\end{array}\right),\left(\begin{array}{c}n \\ j-1\end{array}\right), \ldots,\left(\begin{array}{l}n \\ 1\end{array}\right)\right) \bmod p$ is $p^{1+\left\lfloor\log _{p} j\right\rfloor}$.

Proof. By Lemma 7 the period in $n$ of $\left(\begin{array}{l}n \\ j\end{array}\right) \bmod p$ is $p^{1+\left\lfloor\log _{p} j\right\rfloor}$. Therefore the period in $n$ of the stated set of vectors is the LCM of periods of each, which is also $p^{1+\left\lfloor\log _{p} j\right\rfloor}$.

\subsection{A transform of the prescribed traces problem for $q=2$}

We now transform the problem of counting field elements with prescribed traces to the problem of counting the number of zeros of linear combinations of the trace functions. Note that counting zeros rather than evaluations to one means that one does not necessarily need to introduce a $1 / \bar{n}$ term when defining the relevant curves and as a consequence some results are obtainable for all $n$, rather than just $n$ odd. We first fix some notation, which is virtually the same as that defined in $\S 2.2$.

Let $f_{0}, \ldots, f_{m-1}: \mathbb{F}_{2^{n}} \rightarrow \mathbb{F}_{2}$ be any functions and let $\mathbf{f}=\left(f_{m-1}, \ldots, f_{0}\right)$. For $i, j \in\left\{0, \ldots, 2^{m}-1\right\}$ let $\mathbf{i}=\left(i_{m-1}, \ldots, i_{0}\right)$ and $\mathbf{j}=\left(j_{m-1}, \ldots, j_{0}\right)$ denote the binary expansions of $i$ and $j$ respectively, and let $\mathbf{i} \cdot \mathbf{j}$ denote their inner product $\bmod 2$. For any $i \in\left\{0, \ldots, 2^{m}-1\right\}$, let $\mathbf{i} \cdot \mathbf{f}$ denote the function

$$
\sum_{k=0}^{m-1} i_{k} f_{k}: \mathbb{F}_{2^{n}} \rightarrow \mathbb{F}_{2},
$$

and let $V(\mathbf{i} \cdot \mathbf{f})$ denote the number of zeros in $\mathbb{F}_{2^{n}}$ of $\mathbf{i} \cdot \mathbf{f}$. We interpret $V(\mathbf{0} \cdot \mathbf{f})$ to be the number of zeros of the empty function, which we define to be $2^{n}$. Furthermore, let $N(\mathbf{j})=N\left(j_{m-1}, \ldots, j_{0}\right)$ denote the number of $a \in \mathbb{F}_{2^{n}}$ such that $f_{k}(a)=j_{k}$, for $k=0, \ldots, m-1$.

As before, our goal is to express any $N(\mathbf{j})$ in terms of the $V(\mathbf{i} \cdot \mathbf{f})$, but we begin by first solving the inverse problem, i.e., expressing any $V(\mathbf{i} \cdot \mathbf{f})$ in terms of the $N(\mathbf{j})$.

Lemma 8. With the notation as above, for $0 \leq i \leq 2^{m}-1$ we have

$$
V(\mathbf{i} \cdot \mathbf{f})=\sum_{\mathbf{i} \cdot \mathbf{j}=0} N(\mathbf{j})
$$

Proof. By definition, we have $V(\mathbf{i} \cdot \mathbf{f})=\#\left\{a \in \mathbb{F}_{2^{n}} \mid \mathbf{i} \cdot \mathbf{f}(a)=0\right\}=\#\left\{a \in \mathbb{F}_{2^{n}} \mid \sum_{k=0}^{m-1} i_{k} f_{k}(a)=0\right\}$. Since $N(\mathbf{j})$ counts precisely those $a \in \mathbb{F}_{2^{n}}$ such that $f_{k}(a)=j_{k}$, we must count over all those $\mathbf{j}$ for which $\sum_{k=0}^{m-1} i_{k} f_{k}(a)=0$, i.e., those such that $\sum_{k=0}^{m-1} i_{k} j_{k}=0$. 
Writing Eq. (23) in matrix form, for $i, j \in\left\{0, \ldots, 2^{m}-1\right\}$ we have

$$
[V(\mathbf{i} \cdot \mathbf{f})]^{T}=S_{m} \cdot[N(\mathbf{j})]^{T},
$$

where $\left(S_{m}\right)_{i, j}=1 \mathbf{- i} \cdot \mathbf{j}$ is nothing but Sylvester's construction [48] of Hadamard matrices [24], with the minus ones replaced with zeros. Now let $H_{m}$ be the $2^{m} \times 2^{m}$ matrix with entries $\left(H_{m}\right)_{i, j}=(-1)^{\mathbf{i} \cdot \mathbf{j}}$, i.e., the Walsh-Hadamard transform [54], scaled by $2^{m / 2}$. Since the Walsh-Hadamard matrix is involutory we have $H_{m}^{-1}=\frac{1}{2^{m}} H_{m}$. Also let $A_{m}$ be the $2^{m} \times 2^{m}$ matrix with $\left(A_{m}\right)_{0,0}=1$ and all other entries 0 , and let $B_{m}$ be the $2^{m} \times 2^{m}$ matrix with all entries 1 . Lastly let $\operatorname{Id}_{m}$ be the $2^{m} \times 2^{m}$ identity matrix.

Lemma 9. We have

$$
S_{m}^{-1}=\frac{1}{2^{m-1}} H_{m}-A_{m}
$$

Proof. Noting that $2 S_{m}=H_{m}+B_{m}$, we have

$$
\begin{aligned}
\left(\frac{1}{2^{m-1}} H_{m}-A_{m}\right) S_{m} & =\frac{1}{2^{m}} H_{m} \cdot 2 S_{m}-A_{m} S_{m} \\
& =\frac{1}{2^{m}} H_{m}\left(H_{m}+B_{m}\right)-A_{m} S_{m} \\
& =\operatorname{Id}_{m}+\frac{1}{2^{m}} H_{m} B_{m}-A_{m} S_{m}
\end{aligned}
$$

Since all but the first row of $H_{m}$ contains the same number of ones and minus ones, $\frac{1}{2^{m}} H_{m} B_{m}$ consists of the all-one vector in the first row and the all-zero vector for the others, as does $A_{m} S_{m}$.

Thus in order to compute any of the $2^{m}$ possible outputs $N(\mathbf{j})$ of any set of $m$ functions $\mathbf{f}$, it is sufficient to count the number of zeros of all the $2^{m} \mathbb{F}_{2}$-linear combinations of the functions, and then apply $S_{m}^{-1}$. In particular, one may choose the $f_{0}, \ldots, f_{m-1}$ to be any subset of the trace functions $T_{1}, \ldots, T_{n}$. Note that for $q=2^{r}$ with $r>1$ one must revert to using transform (12).

\subsection{The indirect method}

Let the input traces whose values are prescribed be $\mathbf{f}=\left(T_{l_{m-1}}, \ldots, T_{l_{0}}\right)$ with $l_{m-1}>\cdots>l_{0}$. Then by the transform of the previous subsection, for all $i \in\left\{1, \ldots, 2^{m}-1\right\}$ one needs to compute

$$
V(\mathbf{i} \cdot \mathbf{f})=\#\left\{a \in \mathbb{F}_{2^{n}} \mid \sum_{k=0}^{m-1} i_{k} T_{l_{k}}(a)=0\right\}
$$

In general this problem appears to be non-trivial, since the degree of each $T_{l_{k}}(a)$ in $a$ and its Frobenius powers is $l_{k}$ and the approach of $\S 2$ can not be applied. However, it can be obviated - at least for $n$ odd - by using the following degree-lowering idea. Firstly, note that since the input to the trace functions has linear trace either 0 or 1 , Eq. (25) can be rewritten as

$$
V(\mathbf{i} \cdot \mathbf{f})=\#\left\{a \in \mathbb{F}_{2^{n}} \mid T_{1}(a)=0, \sum_{k=0}^{m-1} i_{k} T_{l_{k}}(a)=0\right\}+\#\left\{a \in \mathbb{F}_{2^{n}} \mid T_{1}(a)=1, \sum_{k=0}^{m-1} i_{k} T_{l_{k}}(a)=0\right\} .
$$

Secondly, note that for $n$ odd Lemma 1 implies

$$
V(\mathbf{i} \cdot \mathbf{f})=\frac{1}{2} \sum_{r_{0} \in \mathbb{F}_{2}} \#\left\{a_{0} \in \mathbb{F}_{2^{n}} \mid \sum_{k=0}^{m-1} i_{k} T_{l_{k}}\left(a_{0}^{2}+a_{0}+r_{0}\right)=0\right\}
$$

Thirdly, it happens that the functions $T_{l}\left(a_{0}^{2}+a_{0}\right)$ and $T_{l}\left(a_{0}^{2}+a_{0}+1\right)$ for $2 \leq l \leq 7$ are all expressible in characteristic two as polynomials of traces of lower degree whose arguments are polynomials in $a_{0}$, 
see §3.4. Hence rather than having a single equation whose zeros one must count (Eq. (25)), one now has two equations whose number of zeros one must add and divide by 2 (Eq. (26)), both now of lower degree than before.

If after the above three steps there are terms that are not linear, i.e., not of the form $T_{1}(\cdot)$ for some argument, then the idea is to pick an argument of a trace function featuring in a non-linear term and apply the above three steps again. In particular, if the chosen argument is $g\left(a_{0}\right)$ then one introduces a new variable $a_{1}$ and as before writes $g\left(a_{0}\right)=a_{1}^{2}+a_{1}+r_{1}$ with $r_{1} \in \mathbb{F}_{2}$ to account for whether the linear trace of $g\left(a_{0}\right)$ is 0 or 1, and expands all those terms in Eq. (26) which have this argument. This results in four equations whose number of zeros one must sum and divide by 4 , with the degrees of the terms which feature this argument having been lowered, as before.

By recursively applying this idea and introducing variables $a_{0}, \ldots, a_{s_{i}-1}$ as necessary, with corresponding linear trace variables $r_{0}, \ldots, r_{s_{i}-1}$, since the degrees of the non-linear terms always decreases one eventually obtains a set of $2^{s_{i}}$ trace equations of the form $T_{1}\left(g_{\mathbf{r}}\left(a_{0}, \ldots, a_{s_{i}-1}\right)\right)=0$ indexed by $\mathbf{r}=\left(r_{0}, \ldots, r_{s_{i}-1}\right) \in\left(\mathbb{F}_{2}\right)^{s_{i}}$, the vector of trace values of the $s_{i}$ rewritten arguments. Each of these can be eliminated by introducing a final variable $a_{s_{i}}$ and writing $a_{s_{i}}^{2}+a_{s_{i}}=g_{\mathbf{r}}\left(a_{0}, \ldots, a_{s_{i}-1}\right)$, giving a system of $s_{i}$ equations in $s_{i}+1$ variables, with the initial variable $a$ having been completely eliminated in going from Eq. (25) to Eq. (26). Observe that since $a_{0}$ is the only free variable in this system, the affine algebraic set that it defines is one-dimensional and is thus a curve.

Depending on $i$, the final linear trace equation features a subset of the coefficients $\left(\begin{array}{l}n \\ l_{k}\end{array}\right)$ for $k=0, \ldots, m-$ 1 , which by Corollary 1 have period a divisor of the maximum period $2^{\theta}:=2^{1+\left\lfloor\log _{2} l_{m-1}\right\rfloor}$. Therefore let $\bar{n} \in\left\{1,3,5, \ldots, 2^{\theta}-1\right\}$ represent the applicable residue classes of $n \bmod 2^{\theta}$ and substitute each $\left(\begin{array}{l}n \\ l_{k}\end{array}\right)$ featuring in the above intersection by $\left(\begin{array}{l}\bar{n} \\ l_{k}\end{array}\right)$. We denote by $C_{i, \mathbf{r}, \bar{n}}$ the affine curve defined by the above $s_{i}$ equations whose coefficients are functions of $\mathbf{r}=\left(r_{0}, \ldots, r_{s_{i}-1}\right)$ and $\bar{n}$. For each $\bar{n}$ we then take the average

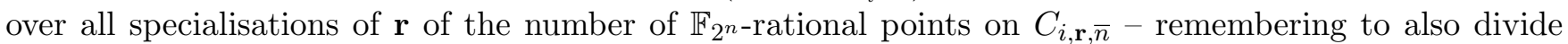
by 2 to account for the presence of $a_{s_{i}}$ - in order to determine $V(\mathbf{i} \cdot \mathbf{f})$, i.e., for all $n \equiv \bar{n}\left(\bmod 2^{\theta}\right)$ with $n \geq l_{m-1}$ we have

$$
V(\mathbf{i} \cdot \mathbf{f})=\frac{1}{2^{s_{i}+1}} \sum_{\mathbf{r} \in\left(\mathbb{F}_{2}\right)^{s_{i}}} \# C_{i, \mathbf{r}, \bar{n}}\left(\mathbb{F}_{2^{n}}\right) .
$$

\subsection{The direct method}

By definition, we have

$$
F_{2}\left(n, t_{l_{0}}, \ldots, t_{l_{m-1}}\right)=\#\left\{a \in \mathbb{F}_{2^{n}} \mid T_{l_{0}}(a)=t_{l_{0}}, \ldots, T_{l_{m-1}}(a)=t_{l_{m-1}}\right\} .
$$

By using the same recursive procedure described in the previous subsection, for $n \geq l_{m-1}$ and odd suppose that for $0 \leq k \leq m-1$ one linearises $T_{l_{k}}(a)$, i.e., reduces it to a $T_{1}$ expression, by introducing variables $a_{0}, a_{k, 1}, \ldots, a_{k, s_{k}-1}$ with corresponding linear traces $r_{0}, r_{k, 1}, \ldots, r_{k, s_{k}-1}$, resulting in $2^{s_{k}}$ trace equations of the form

$$
T_{1}\left(g_{\mathbf{r}_{k}}\left(a_{0}, a_{k, 1}, \ldots, a_{k, s_{k}-1}\right)\right)=t_{l_{k}} .
$$

Observe that amongst all of the $a_{k, i}$ 's there may be definitional repetitions. Therefore, let $U$ be the union of all of the equations arising from the linearisation of each of the featured trace functions, once repetitions have been eliminated. Let $s \leq \sum_{k=0}^{m-1} s_{k}$ be the number of variables in this union, which we relabel as $a_{0}, a_{1}, \ldots, a_{s-1}$, and let $\mathbf{r}=\left(r_{0}, \ldots, r_{s-1}\right)$ be the corresponding vector of possible linear traces of the rewritten arguments. Note that since $a$ was completely eliminated by the introduction of $a_{0}$ there are only $s-1$ corresponding equations in addition to the $m$ trace conditions in (28). Each of these $m$ trace equations can be parameterised by introducing for $0 \leq k \leq m-1$ a variable $a_{s_{k}}$ and writing $a_{s_{k}}^{2}+a_{s_{k}}+t_{l_{k}}=g_{\mathbf{r}_{k}}\left(a_{0}, a_{1} \ldots, a_{s-1}\right)$, giving a system of $m+s-1$ equations in $m+s$ variables. As in the indirect method observe that $a_{0}$ is the only free variable in this system and so the affine algebraic set that it defines is one-dimensional and is thus a curve. 
Also as before, this system features the coefficients $\left(\begin{array}{c}n \\ l_{k}\end{array}\right)$, which by Corollary 1 have period a divisor of $2^{\theta}$, so again let $\bar{n} \in\left\{1,3,5, \ldots, 2^{\theta}-1\right\}$ represent the applicable residue classes of $n \bmod 2^{\theta}$, and substitute each $\left(\begin{array}{l}n \\ l_{k}\end{array}\right)$ by $\left(\begin{array}{l}\bar{n} \\ l_{k}\end{array}\right)$. We denote by $C_{\mathbf{r}, \bar{n}}$ the affine curve defined by the $m+s-1$ above equations whose coefficients are functions of the prescribed values, $\mathbf{r}=\left(r_{0}, \ldots, r_{s-1}\right)$ and $\bar{n}$. For each $\bar{n}$ we take the average over all specialisations of $\mathbf{r}$ of the number of $\mathbb{F}_{2^{n}}$-rational points on $C_{\mathbf{r}, \bar{n}}$ and divide by $2^{m}$ in order to determine $F_{2}\left(n, t_{l_{0}}, \ldots, t_{l_{m-1}}\right)$, i.e., for all $n \equiv \bar{n}\left(\bmod 2^{\theta}\right)$ with $n \geq l_{m-1}$ we have

$$
F_{2}\left(n, t_{l_{0}}, \ldots, t_{l_{m-1}}\right)=\frac{1}{2^{m+s}} \sum_{\mathbf{r} \in\left(\mathbb{F}_{2}\right)^{s}} \# C_{\mathbf{r}, \bar{n}}\left(\mathbb{F}_{2^{n}}\right) .
$$

Since in general there will be more variables and equations than for the indirect method, computing the zeta functions of the arising curves will likely be more costly for this method.

\subsection{Computing $T_{l}(\alpha-\beta)$}

We now explain the how one can obtain expressions for $T_{l}\left(a_{0}^{2}+a_{0}\right)$ and $T_{l}\left(a_{0}^{2}+a_{0}+1\right)$ for $2 \leq l \leq 7$. Expressions for $T_{2}(\alpha-\beta)$ and $T_{3}(\alpha-\beta)$ in characteristic two were given by Fitgerald and Yucas [17, Lemma 1.1] and proven by expanding the bilinear forms $T_{l}(\alpha+\beta)+T_{l}(\alpha)+T_{l}(\beta)$ in terms of the Frobenius powers of $\alpha$ and $\beta$, and deducing the correct function of the lower degree traces. It is possible - though laborious - to continue in this manner (we did so for $l=4$ ), so instead we present an easier method.

We begin by recalling Eq (3):

$$
k T_{k}(\alpha)=\sum_{j=1}^{k}(-1)^{j-1} T_{l-j}(\alpha) T_{1}\left(\alpha^{j}\right) .
$$

In order to use the argument $\alpha-\beta$ we need to work instead in the ring $\mathbb{Z}\left[\alpha_{1}, \ldots, \alpha_{n}, \beta_{1}, \ldots, \beta_{n}\right]$ and with the ring of multisymmetric functions in two variables, with the symmetric group $S_{n}$ acting on $\alpha_{1}, \ldots, \alpha_{n}$ and $\beta_{1}, \ldots, \beta_{n}$ independently (see [12] for a formal definition). Abusing notation slightly, in this ring Eq. (3) becomes:

$$
l T_{l}(\alpha-\beta)=\sum_{k=1}^{l}(-1)^{k-1} T_{l-k}(\alpha-\beta) T_{1}\left((\alpha-\beta)^{k}\right) .
$$

If one works over $\mathbb{Q}$ rather than $\mathbb{Z}$ then Eq. (30) leads to expressions for $T_{l}(\alpha-\beta)$ for any $l \geq 1$ as a sum of products of $T_{1}$ terms with arguments being various powers of $\alpha-\beta$. However, in positive characteristic this is not so useful. For instance, computing $T_{2}(\alpha-\beta)$ in characteristic two in this way is not possible as the left hand side vanishes. Nevertheless, working inductively for $2 \leq l \leq 7$ and applying Newton's identities evaluated at various products of powers of $\alpha$ and $\beta$ so that no trace occurs to any power larger than one, all of the coefficients become divisible by $l$. Upon dividing by $l$ one obtains an equation for $T_{l}(\alpha-\beta)$ over $\mathbb{Z}$, which can then be substituted into Eq. (30) in order to attempt to compute $T_{l+1}(\alpha-\beta)$. For example, from Eq. (30) we have

$$
\begin{aligned}
2 T_{2}(\alpha-\beta) & =T_{1}(\alpha-\beta)^{2}-T_{1}\left((\alpha-\beta)^{2}\right) \\
& =\left(T_{1}(\alpha)-T_{1}(\beta)\right)^{2}-T_{1}\left(\alpha^{2}\right)+2 T_{1}(\alpha \beta)-T_{1}\left(\beta^{2}\right) \\
& =T_{1}(\alpha)^{2}-2 T_{1}(\alpha) T_{1}(\beta)+T_{1}(\beta)^{2}-T_{1}\left(\alpha^{2}\right)+2 T_{1}(\alpha \beta)-T_{1}\left(\beta^{2}\right) \\
& =2 T_{2}(\alpha)+2 T_{2}(\beta)-2 T_{1}(\alpha) T_{1}(\beta)+2 T_{1}(\alpha \beta),
\end{aligned}
$$

where in the final line we have used Eq. (3) for $l=2$ for $\alpha$ and $\beta$ separately. We have therefore proven that

$$
T_{2}(\alpha-\beta)=T_{2}(\alpha)+T_{2}(\beta)-T_{1}(\alpha) T_{1}(\beta)+T_{1}(\alpha \beta) .
$$

The parts of the following lemma can either be proven by induction on $n$ using the identity

$$
T_{l}\left(\alpha_{1}, \ldots, \alpha_{n}\right)=T_{l-1}\left(\alpha_{1}, \ldots, \alpha_{n-1}\right) \alpha_{n}+T_{l}\left(\alpha_{1}, \ldots, \alpha_{n-1}\right),
$$

or via sequences of manipulations as described above ${ }^{\dagger}$.

\footnotetext{
${ }^{\dagger}$ See NewtonApproach_1_le_7.mw for a derivation of these expressions.
} 
Lemma 10. For all $n \geq l$ we have

(1) $T_{1}(\alpha-\beta)=T_{1}(\alpha)-T_{1}(\beta)$,

(2) $T_{2}(\alpha-\beta)=T_{2}(\alpha)+T_{2}(\beta)-T_{1}(\alpha) T_{1}(\beta)+T_{1}(\alpha \beta)$,

(3) $T_{3}(\alpha-\beta)=T_{3}(\alpha)-T_{3}(\beta)+T_{1}(\alpha) T_{2}(\beta)-T_{1}(\beta) T_{2}(\alpha)+T_{1}(\alpha) T_{1}(\alpha \beta)-T_{1}(\beta) T_{1}(\alpha \beta)$ $+T_{1}\left(\alpha \beta^{2}\right)-T_{1}\left(\alpha^{2} \beta\right)$

(4) $T_{4}(\alpha-\beta)=T_{4}(\alpha)+T_{4}(\beta)-T_{1}(\alpha) T_{3}(\beta)-T_{1}(\beta) T_{3}(\alpha)+T_{2}(\alpha) T_{2}(\beta)-T_{1}(\alpha) T_{1}(\beta) T_{1}(\alpha \beta)$ $+T_{1}(\alpha \beta) T_{2}(\alpha)+T_{1}(\alpha \beta) T_{2}(\beta)-T_{1}(\alpha) T_{1}\left(\alpha^{2} \beta\right)+T_{1}(\alpha) T_{1}\left(\alpha \beta^{2}\right)+T_{1}(\beta) T_{1}\left(\alpha^{2} \beta\right)$

$-T_{1}(\beta) T_{1}\left(\alpha \beta^{2}\right)+T_{1}\left(\alpha^{3} \beta\right)-T_{1}\left(\alpha^{2} \beta^{2}\right)+T_{1}\left(\alpha \beta^{3}\right)+T_{2}(\alpha \beta)$

(5) $T_{5}(\alpha-\beta)=T_{5}(\alpha)-T_{5}(\beta)+T_{1}(\alpha) T_{4}(\beta)-T_{1}(\beta) T_{4}(\alpha)+T_{2}(\beta) T_{3}(\alpha)-T_{2}(\alpha) T_{3}(\beta)+T_{1}(\alpha) T_{1}(\beta) T_{1}\left(\alpha^{2} \beta\right)$

$-T_{1}(\beta) T_{1}(\alpha \beta) T_{2}(\alpha)+T_{1}(\alpha) T_{1}(\alpha \beta) T_{2}(\beta)-T_{1}(\alpha) T_{1}(\beta) T_{1}\left(\alpha \beta^{2}\right)-T_{1}\left(\alpha^{2} \beta\right) T_{2}(\alpha)$

$-T_{1}\left(\alpha^{2} \beta\right) T_{2}(\beta)+T_{1}\left(\alpha \beta^{2}\right) T_{2}(\alpha)+T_{1}\left(\alpha \beta^{2}\right) T_{2}(\beta)+T_{1}(\alpha \beta) T_{3}(\alpha)-T_{1}(\alpha \beta) T_{3}(\beta)$

$-T_{1}(\alpha \beta) T_{1}\left(\alpha^{2} \beta\right)+T_{1}(\alpha \beta) T_{1}\left(\alpha \beta^{2}\right)+T_{1}(\alpha) T_{1}\left(\alpha^{3} \beta\right)-T_{1}(\alpha) T_{1}\left(\alpha^{2} \beta^{2}\right)+T_{1}(\alpha) T_{1}\left(\alpha \beta^{3}\right)$

$-T_{1}(\beta) T_{1}\left(\alpha^{3} \beta\right)+T_{1}(\beta) T_{1}\left(\alpha^{2} \beta^{2}\right)-T_{1}(\beta) T_{1}\left(\alpha \beta^{3}\right)+T_{1}(\alpha) T_{2}(\alpha \beta)-T_{1}(\beta) T_{2}(\alpha \beta)$

$-T_{1}\left(\alpha^{4} \beta\right)+2 T_{1}\left(\alpha^{3} \beta^{2}\right)-2 T_{1}\left(\alpha^{2} \beta^{3}\right)+T_{1}\left(\alpha \beta^{4}\right)$,

(6) $T_{6}(\alpha-\beta)=T_{6}(\alpha)+T_{6}(\beta)-T_{1}(\alpha) T_{5}(\beta)-T_{1}(\beta) T_{5}(\alpha)+T_{2}(\alpha) T_{4}(\beta)+T_{2}(\beta) T_{4}(\alpha)-T_{3}(\beta) T_{3}(\alpha)$

$+T_{2}(\alpha) T_{2}(\alpha \beta)+T_{1}\left(\alpha^{5} \beta\right)-2 T_{1}\left(\alpha^{4} \beta^{2}\right)+2 T_{1}\left(\alpha^{3} \beta^{3}\right)-2 T_{1}\left(\alpha^{2} \beta^{4}\right)+T_{1}\left(\alpha \beta^{5}\right)$

$+T_{1}(\alpha \beta) T_{2}(\alpha) T_{2}(\beta)+T_{2}(\alpha \beta) T_{2}(\beta)-T_{1}(\alpha) T_{1}(\alpha \beta) T_{1}\left(\alpha^{2} \beta\right)-T_{1}(\beta) T_{1}(\alpha \beta) T_{1}\left(\beta^{2} \alpha\right)$

$-T_{1}(\alpha) T_{1}(\beta) T_{2}(\alpha \beta)+T_{1}(\beta) T_{1}\left(\alpha^{2} \beta\right) T_{2}(\alpha)+T_{1}(\alpha) T_{1}\left(\alpha \beta^{2}\right) T_{2}(\beta)-T_{1}(\alpha) T_{1}(\beta) T_{1}\left(\alpha \beta^{3}\right)$

$+T_{1}(\alpha) T_{1}(\beta) T_{1}\left(\alpha^{2} \beta^{2}\right)+T_{1}(\alpha) T_{1}(\alpha \beta) T_{1}\left(\alpha \beta^{2}\right)-T_{1}(\beta) T_{1}\left(\alpha \beta^{2}\right) T_{2}(\alpha)-T_{1}(\alpha) T_{1}(\beta) T_{1}\left(\alpha^{3} \beta\right)$

$-T_{1}(\beta) T_{1}(\alpha \beta) T_{3}(\alpha)-T_{1}(\alpha) T_{1}\left(\alpha^{2} \beta\right) T_{2}(\beta)-T_{1}(\alpha) T_{1}(\alpha \beta) T_{3}(\beta)+T_{1}(\beta) T_{1}(\alpha \beta) T_{1}\left(\alpha^{2} \beta\right)$

$+4 T_{3}(\alpha \beta)+T_{2}\left(\alpha \beta^{2}\right)-T_{1}(\alpha) T_{1}\left(\alpha^{4} \beta\right)+2 T_{1}(\alpha) T_{1}\left(\alpha^{3} \beta^{2}\right)-2 T_{1}(\alpha) T_{1}\left(\alpha^{2} \beta^{3}\right)+T_{1}(\alpha) T_{1}\left(\alpha \beta^{4}\right)$

$+T_{1}(\beta) T_{1}\left(\alpha^{4} \beta\right)-2 T_{1}(\beta) T_{1}\left(\alpha^{3} \beta^{2}\right)+2 T_{1}(\beta) T_{1}\left(\alpha^{2} \beta^{3}\right)-T_{1}(\beta) T_{1}\left(\alpha \beta^{4}\right)+T_{2}\left(\alpha^{2} \beta\right)$

$+T_{1}(\alpha \beta) T_{4}(\alpha)+T_{1}(\alpha \beta) T_{4}(\beta)+T_{1}(\alpha \beta) T_{1}\left(\alpha^{3} \beta\right)+T_{1}(\alpha \beta) T_{1}\left(\alpha \beta^{3}\right)-T_{1}(\alpha \beta) T_{2}(\alpha \beta)$

$-T_{1}\left(\alpha^{2} \beta\right) T_{3}(\alpha)+T_{1}\left(\alpha^{2} \beta\right) T_{3}(\beta)-T_{1}\left(\alpha^{2} \beta\right) T_{1}\left(\alpha \beta^{2}\right)+T_{1}\left(\alpha \beta^{2}\right) T_{3}(\alpha)-T_{1}\left(\alpha \beta^{2}\right) T_{3}(\beta)$

$+T_{1}\left(\alpha^{3} \beta\right) T_{2}(\alpha)+T_{1}\left(\alpha^{3} \beta\right) T_{2}(\beta)-T_{1}\left(\alpha^{2} \beta^{2}\right) T_{2}(\alpha)-T_{1}\left(\alpha^{2} \beta^{2}\right) T_{2}(\beta)+T_{1}\left(\alpha \beta^{3}\right) T_{2}(\alpha)$

$+T_{1}\left(\alpha \beta^{3}\right) T_{2}(\beta)$

(7) $T_{7}(\alpha-\beta)=T_{7}(\alpha)-T_{7}(\beta)+T_{1}(\alpha) T_{6}(\beta)-T_{6}(\alpha) T_{1}(\beta)-T_{2}(\alpha) T_{5}(\beta)+T_{2}(\beta) T_{5}(\alpha)+T_{3}(\alpha) T_{4}(\beta)$

$-T_{3}(\beta) T_{4}(\alpha)+T_{1}(\alpha \beta) T_{5}(\alpha)-T_{1}(\alpha \beta) T_{5}(\beta)+T_{1}\left(\alpha \beta^{6}\right)-T_{1}\left(\alpha^{6} \beta\right)-T_{1}(\alpha \beta) T_{1}\left(\alpha^{4} \beta\right)$

$+2 T_{1}(\alpha \beta) T_{1}\left(\alpha^{3} \beta^{2}\right)-2 T_{1}(\alpha \beta) T_{1}\left(\alpha^{2} \beta^{3}\right)+T_{1}(\alpha \beta) T_{1}\left(\alpha \beta^{4}\right)-T_{1}\left(\alpha^{2} \beta\right) T_{1}\left(\alpha^{3} \beta\right)$

$+T_{1}\left(\alpha^{2} \beta\right) T_{1}\left(\alpha^{2} \beta^{2}\right)-T_{1}\left(\alpha^{2} \beta\right) T_{1}\left(\alpha \beta^{3}\right)-T_{2}(\alpha \beta) T_{3}(\beta)-T_{1}(\beta) T_{1}\left(\alpha \beta^{2}\right) T_{3}(\alpha)$

$-T_{1}(\beta) T_{1}\left(\alpha^{3} \beta\right) T_{2}(\alpha)+T_{1}(\beta) T_{1}\left(\alpha^{2} \beta^{2}\right) T_{2}(\alpha)-T_{1}(\beta) T_{2}(\alpha \beta) T_{2}(\alpha)-T_{1}(\beta) T_{1}\left(\alpha \beta^{3}\right) T_{2}(\alpha)$

$+3 T_{1}\left(\alpha^{5} \beta^{2}\right)-5 T_{1}\left(\alpha^{4} \beta^{3}\right)+5 T_{1}\left(\alpha^{3} \beta^{4}\right)-3 T_{1}\left(\alpha^{2} \beta^{5}\right)+T_{2}(\alpha \beta) T_{3}(\alpha)+T_{1}(\alpha) T_{1}(\alpha \beta) T_{1}\left(\alpha^{3} \beta\right)$

$+2 T_{1}(\alpha) T_{1}(\alpha \beta) T_{1}\left(\alpha^{2} \beta^{2}\right)+T_{1}(\alpha) T_{1}(\alpha \beta) T_{1}\left(\alpha \beta^{3}\right)+T_{1}(\alpha) T_{1}(\alpha \beta) T_{4}(\beta)$

$-3 T_{1}(\alpha) T_{1}(\alpha \beta) T_{2}(\alpha \beta)+T_{1}(\alpha) T_{1}\left(\alpha^{2} \beta\right) T_{3}(\beta)-T_{1}(\alpha) T_{1}\left(\alpha^{2} \beta\right) T_{1}\left(\alpha \beta^{2}\right)$

$-T_{1}(\alpha) T_{1}\left(\alpha \beta^{2}\right) T_{3}(\beta)+T_{1}(\alpha) T_{1}\left(\alpha^{3} \beta\right) T_{2}(\beta)-T_{1}(\alpha) T_{1}\left(\alpha^{2} \beta^{2}\right) T_{2}(\beta)+T_{1}(\alpha) T_{1}(\beta) T_{1}\left(\alpha^{4} \beta\right)$

$-2 T_{1}(\alpha) T_{1}(\beta) T_{1}\left(\alpha^{3} \beta^{2}\right)+2 T_{1}(\alpha) T_{1}(\beta) T_{1}\left(\alpha^{2} \beta^{3}\right)-T_{1}(\alpha) T_{1}(\beta) T_{1}\left(\alpha \beta^{4}\right)$

$+T_{1}(\alpha) T_{2}(\alpha \beta) T_{2}(\beta)+T_{1}(\alpha) T_{1}\left(\alpha \beta^{3}\right) T_{2}(\beta)-T_{1}(\beta) T_{1}(\alpha \beta) T_{1}\left(\alpha^{3} \beta\right)$

$-2 T_{1}(\beta) T_{1}(\alpha \beta) T_{1}\left(\alpha^{2} \beta^{2}\right)-T_{1}(\beta) T_{1}(\alpha \beta) T_{1}\left(\alpha \beta^{3}\right)-T_{1}(\beta) T_{1}(\alpha \beta) T_{4}(\alpha)$

$+3 T_{1}(\beta) T_{1}(\alpha \beta) T_{2}(\alpha \beta)+T_{1}(\beta) T_{1}\left(\alpha^{2} \beta\right) T_{3}(\alpha)+T_{1}(\beta) T_{1}\left(\alpha^{2} \beta\right) T_{1}\left(\alpha \beta^{2}\right)$

$-T_{1}\left(\alpha^{2} \beta\right) T_{2}(\beta) T_{2}(\alpha)+T_{1}\left(\alpha \beta^{2}\right) T_{2}(\beta) T_{2}(\alpha)+T_{1}(\alpha \beta) T_{2}(\beta) T_{3}(\alpha)+T_{1}(\alpha) T_{2}\left(\alpha \beta^{2}\right)$ 


$$
\begin{aligned}
& +T_{1}(\alpha) T_{2}\left(\alpha^{2} \beta\right)+10 T_{1}(\alpha) T_{3}(\alpha \beta)-T_{1}(\beta) T_{2}\left(\alpha \beta^{2}\right)-T_{1}(\beta) T_{2}\left(\alpha^{2} \beta\right)-10 T_{1}(\beta) T_{3}(\alpha \beta) \\
& +T_{1}(\alpha) T_{1}\left(\alpha^{5} \beta\right)-2 T_{1}(\alpha) T_{1}\left(\alpha^{4} \beta^{2}\right)-2 T_{1}(\alpha) T_{1}\left(\alpha^{2} \beta^{4}\right)+T_{1}(\alpha) T_{1}\left(\alpha \beta^{5}\right)-T_{1}(\beta) T_{1}\left(\alpha^{5} \beta\right) \\
& +2 T_{1}(\beta) T_{1}\left(\alpha^{4} \beta^{2}\right)+2 T_{1}(\beta) T_{1}\left(\alpha^{2} \beta^{4}\right)-T_{1}(\beta) T_{1}\left(\alpha \beta^{5}\right)-T_{1}\left(\alpha^{4} \beta\right) T_{2}(\alpha)-T_{1}\left(\alpha^{4} \beta\right) T_{2}(\beta) \\
& +2 T_{1}\left(\alpha^{3} \beta^{2}\right) T_{2}(\alpha)+2 T_{1}\left(\alpha^{3} \beta^{2}\right) T_{2}(\beta)-2 T_{1}\left(\alpha^{2} \beta^{3}\right) T_{2}(\alpha)-2 T_{1}\left(\alpha^{2} \beta^{3}\right) T_{2}(\beta) \\
& +T_{1}\left(\alpha \beta^{4}\right) T_{2}(\alpha)+T_{1}\left(\alpha \beta^{4}\right) T_{2}(\beta)+T_{1}\left(\alpha^{3} \beta\right) T_{3}(\alpha)-T_{1}\left(\alpha^{3} \beta\right) T_{3}(\beta)-T_{1}\left(\alpha^{2} \beta^{2}\right) T_{3}(\alpha) \\
& +T_{1}\left(\alpha^{2} \beta^{2}\right) T_{3}(\beta)+T_{1}\left(\alpha \beta^{3}\right) T_{3}(\alpha)-T_{1}\left(\alpha \beta^{3}\right) T_{3}(\beta)-T_{1}\left(\alpha^{2} \beta\right) T_{4}(\alpha)-T_{1}\left(\alpha^{2} \beta\right) T_{4}(\beta) \\
& -T_{1}\left(\alpha^{2} \beta\right) T_{2}(\alpha \beta)+T_{1}\left(\alpha \beta^{2}\right) T_{1}\left(\alpha^{3} \beta\right)-T_{1}\left(\alpha \beta^{2}\right) T_{1}\left(\alpha^{2} \beta^{2}\right)+T_{1}\left(\alpha \beta^{2}\right) T_{1}\left(\alpha \beta^{3}\right) \\
& +T_{1}\left(\alpha \beta^{2}\right) T_{4}(\alpha)+T_{1}\left(\alpha \beta^{2}\right) T_{4}(\beta)+T_{1}\left(\alpha \beta^{2}\right) T_{2}(\alpha \beta)-T_{1}(\alpha \beta) T_{1}\left(\alpha^{2} \beta\right) T_{2}(\alpha) \\
& -T_{1}(\alpha \beta) T_{1}\left(\alpha^{2} \beta\right) T_{2}(\beta)+T_{1}(\alpha \beta) T_{1}\left(\alpha \beta^{2}\right) T_{2}(\alpha)+T_{1}(\alpha \beta) T_{1}\left(\alpha \beta^{2}\right) T_{2}(\beta) \\
& -T_{1}(\alpha \beta) T_{2}(\alpha) T_{3}(\beta)+T_{1}(\alpha) T_{1}(\beta) T_{1}(\alpha \beta) T_{1}\left(\alpha^{2} \beta\right)-T_{1}(\alpha) T_{1}(\beta) T_{1}(\alpha \beta) T_{1}\left(\alpha \beta^{2}\right) .
\end{aligned}
$$

Observe that for each $1 \leq l \leq 7$ all of the terms appearing in Lemma 10 part (l) have total degree $l$, when counted in the natural way. Hence when one reduces $\bmod 2$ and sets $\beta=\alpha^{2}$, the two terms $T_{l}(\alpha)$ and $T_{l}(\beta)$ cancel, leaving only $T_{l}$ 's of degree $<l$, as claimed earlier.

Unfortunately, our approach of using Newton's identities evaluated for various $l$ at products of powers of $\alpha$ and $\beta$ so that no trace occurs to any power larger than one, fails for $l=8$ due to the presence of the term $T_{2}(x y)^{2}$, which can not be eliminated while keeping the remaining terms' coefficients divisible by 8 . Whether or not there exist such expressions for $T_{l}(\alpha-\beta)$ over $\mathbb{Z}$ for $l \geq 8$, we leave as an open problem. Note that it is known that the ring of multisymmetric functions in two sets of variables $\alpha_{1}, \ldots, \alpha_{n}$ and $\beta_{1}, \ldots, \beta_{n}$ is not generated over $\mathbb{Z}$ by the elementary multisymmetric functions that we are using, unless $n=2[12]$. However, since we are only interested in a particular family of multisymmetric functions namely $T_{l}(\alpha-\beta)$ - and not all of them, it is possible that such expressions exist.

\section{Curves and Explicit Formulae for $q=2$ and $l \leq 7$}

In this section we apply the indirect method and for some cases the direct method to determine relevant curves for $l \leq 7$ and $n$ odd. Using Magma we provide explicit formulae for $l \leq 5$ and $n$ odd using the indirect method, and provide explicit formulae for a subset of these cases using the direct method, for all relevant $n$.

In practice, rather than obtain a curve as sketched in the previous section for each $i \in\left\{0, \ldots, 2^{l}-1\right\}$, it is more efficient to compute a linearisation for each featured $T_{l_{k}}(a)$ and then combine them as appropriate according to whether $i_{k}$ is 0 or 1 for a given $i$, as we do in the examples that follow. Observe that for the indirect method, once $V(\mathbf{i} \cdot \mathbf{f})$ has been obtained for $\mathbf{f}=\left(T_{l}, \ldots, T_{1}\right)$ and $i \in\left\{0, \ldots, 2^{l}-1\right\}$, these functions need not be recomputed for the subsequent $\mathbf{f}=\left(T_{l+1}, \ldots, T_{1}\right)$.

\subsection{Computing $F_{2}\left(n, t_{1}, t_{2}, t_{3}\right)$}

The formulae for $l=3$ were presented in $[3, \S \S 4 \& 5]$ - although obtained there in a slightly different manner - but we include them here for demonstration purposes and completeness.

4.1.1 Indirect method. Setting $\mathbf{f}=\left(T_{3}, T_{2}, T_{1}\right)$, by the transform (24) we have

$$
\left[\begin{array}{l}
F_{2}(n, 0,0,0) \\
F_{2}(n, 1,0,0) \\
F_{2}(n, 0,1,0) \\
F_{2}(n, 1,1,0) \\
F_{2}(n, 0,0,1) \\
F_{2}(n, 1,0,1) \\
F_{2}(n, 0,1,1) \\
F_{2}(n, 1,1,1)
\end{array}\right]=\left[\begin{array}{l}
N(\mathbf{0}) \\
N(\mathbf{1}) \\
N(\mathbf{2}) \\
N(\mathbf{3}) \\
N(\mathbf{4}) \\
N(\mathbf{5}) \\
N(\mathbf{6}) \\
N(\mathbf{7})
\end{array}\right]=\frac{1}{4}\left[\begin{array}{rrrrrrrr}
-3 & 1 & 1 & 1 & 1 & 1 & 1 & 1 \\
1 & -1 & 1 & -1 & 1 & -1 & 1 & -1 \\
1 & 1 & -1 & -1 & 1 & 1 & -1 & -1 \\
1 & -1 & -1 & 1 & 1 & -1 & -1 & 1 \\
1 & 1 & 1 & 1 & -1 & -1 & -1 & -1 \\
1 & -1 & 1 & -1 & -1 & 1 & -1 & 1 \\
1 & 1 & -1 & -1 & -1 & -1 & 1 & 1 \\
1 & -1 & -1 & 1 & -1 & 1 & 1 & -1
\end{array}\right]\left[\begin{array}{l}
V(\mathbf{0} \cdot \mathbf{f}) \\
V(\mathbf{1} \cdot \mathbf{f}) \\
V(\mathbf{2} \cdot \mathbf{f}) \\
V(\mathbf{3} \cdot \mathbf{f}) \\
V(\mathbf{4} \cdot \mathbf{f}) \\
V(\mathbf{5} \cdot \mathbf{f}) \\
V(\mathbf{6} \cdot \mathbf{f}) \\
V(\mathbf{7} \cdot \mathbf{f})
\end{array}\right]
$$


By definition we have $V(\mathbf{0} \cdot \mathbf{f})=2^{n}$, while $V(\mathbf{1} \cdot \mathbf{f})=V\left(T_{1}\right)=\#\left\{a \in \mathbb{F}_{2^{n}} \mid T_{1}(a)=0\right\}=2^{n-1}$. To determine $V(\mathbf{i} \cdot \mathbf{f})$ for $2 \leq i \leq 7$, we use Lemma 10 parts (1) to (3). In particular, setting $\alpha=a_{0}^{2}$ and $\beta=a_{0}$ for $r_{0}=0$, and $\alpha=a_{0}^{2}+a_{0}$ and $\beta=1$ for $r_{0}=1$, and evaluating $\bmod 2$ gives the following:

$$
\begin{aligned}
& T_{1}\left(a_{0}^{2}+a_{0}+r_{0}\right)=T_{1}\left(r_{0}\right), \\
& T_{2}\left(a_{0}^{2}+a_{0}+r_{0}\right)=T_{1}\left(a_{0}^{3}+a_{0}+r_{0}\left(\begin{array}{c}
n \\
2
\end{array}\right)\right), \\
& T_{3}\left(a_{0}^{2}+a_{0}+r_{0}\right)=T_{1}\left(a_{0}^{5}+a_{0}+r_{0}\left(a_{0}^{3}+a_{0}+\left(\begin{array}{c}
n \\
3
\end{array}\right)\right)\right) .
\end{aligned}
$$

For $2 \leq i \leq 7$ let $\mathbf{i}=\left(i_{2}, i_{1}, i_{0}\right)$. The curves we are interested in for $a$ of trace $r_{0}$ are therefore:

$$
a_{1}^{2}+a_{1}=i_{2}\left(a_{0}^{5}+a_{0}+r_{0}\left(a_{0}^{3}+a_{0}+\left(\begin{array}{l}
n \\
3
\end{array}\right)\right)\right)+i_{1}\left(a_{0}^{3}+a_{0}+r_{0}\left(\begin{array}{l}
n \\
2
\end{array}\right)\right)+i_{0} r_{0}\left(\begin{array}{l}
n \\
1
\end{array}\right) .
$$

These curves have genus 1 if $i_{2}=0$ and genus 2 if $i_{2}=1$, and are all supersingular. As pointed out in [3], this is why the formulae are periodic in $n$.

By Corollary 1 the vector $\left(\left(\begin{array}{l}n \\ 3\end{array}\right),\left(\begin{array}{l}n \\ 2\end{array}\right),\left(\begin{array}{l}n \\ 1\end{array}\right)\right) \bmod 2$ has period 4 and is equal to $(0,0,1)$ if $n \equiv 1(\bmod 4)$, and $(1,1,1)$ if $n \equiv 3(\bmod 4)$. Hence there are two cases to consider when computing the zeta functions of the curves specified in Eq. (35). In order to express $F_{2}\left(n, t_{1}, t_{2}, t_{3}\right)$ compactly, we define the following polynomials:

$$
\begin{aligned}
& \delta_{2,1}=X^{2}+2 X+2, \\
& \delta_{4,1}=X^{4}+2 X^{3}+2 X^{2}+4 X+4,
\end{aligned}
$$

which are the characteristic polynomials of Frobenius of the following two supersingular curves, respectively:

$$
\begin{aligned}
& C_{2,1} / \mathbb{F}_{2}: y^{2}+y=x^{3}+x, \\
& C_{4,1} / \mathbb{F}_{2}: y^{2}+y=x^{5}+x^{3} .
\end{aligned}
$$

Using Magma to compute the zeta functions of the curves (35) and applying (31) gives the following.

Theorem 6. For $n \geq 3$ we have

$$
\begin{aligned}
& F_{2}(n, 0,0,0)=2^{n-3}-\frac{1}{8}\left(2 \rho_{n}\left(\delta_{2,1}\right)+\rho_{n}\left(\delta_{4,1}\right)\right) \quad \text { if } n \equiv 1,3 \quad(\bmod 4) \\
& F_{2}(n, 1,0,0)=2^{n-3}-\frac{1}{8} \cdot\left\{\begin{array}{lll}
2 \rho_{n}\left(\delta_{2,1}\right)+\rho_{n}\left(\delta_{4,1}\right) & \text { if } n \equiv 1 & (\bmod 4) \\
-\rho_{n}\left(\delta_{4,1}\right) & \text { if } n \equiv 3 & (\bmod 4)
\end{array}\right. \\
& F_{2}(n, 0,1,0)=2^{n-3}-\frac{1}{8}\left(-\rho_{n}\left(\delta_{4,1}\right)\right) \text { if } n \equiv 1,3 \quad(\bmod 4) \\
& F_{2}(n, 1,1,0)=2^{n-3}-\frac{1}{8} \cdot\left\{\begin{array}{lll}
-2 \rho_{n}\left(\delta_{2,1}\right)+\rho_{n}\left(\delta_{4,1}\right) & \text { if } n \equiv 1 & (\bmod 4) \\
-\rho_{n}\left(\delta_{4,1}\right) & \text { if } n \equiv 3 & (\bmod 4)
\end{array}\right. \\
& F_{2}(n, 0,0,1)=2^{n-3}-\frac{1}{8}\left(-\rho_{n}\left(\delta_{4,1}\right)\right) \quad \text { if } n \equiv 1,3 \quad(\bmod 4) \\
& F_{2}(n, 1,0,1)=2^{n-3}-\frac{1}{8} \cdot\left\{\begin{array}{lll}
-\rho_{n}\left(\delta_{4,1}\right) & \text { if } n \equiv 1 \quad(\bmod 4) \\
-2 \rho_{n}\left(\delta_{2,1}\right)+\rho_{n}\left(\delta_{4,1}\right) & \text { if } n \equiv 3 \quad(\bmod 4)
\end{array}\right. \\
& F_{2}(n, 0,1,1)=2^{n-3}-\frac{1}{8}\left(-2 \rho_{n}\left(\delta_{2,1}\right)+\rho_{n}\left(\delta_{4,1}\right)\right) \quad \text { if } n \equiv 1,3 \quad(\bmod 4) \\
& F_{2}(n, 1,1,1)=2^{n-3}-\frac{1}{8} \cdot\left\{\begin{array}{lll}
-\rho_{n}\left(\delta_{4,1}\right) & \text { if } n \equiv 1 & (\bmod 4) \\
2 \rho_{n}\left(\delta_{2,1}\right)+\rho_{n}\left(\delta_{4,1}\right) & \text { if } n \equiv 3 & (\bmod 4)
\end{array}\right.
\end{aligned}
$$

The roots of $\delta_{2,1}$ are $\sqrt{2} \omega_{8}^{3}, \sqrt{2} \omega_{8}^{5}$, with $\omega_{8}=e^{i \pi / 4}=(1+i) / \sqrt{2}$, while the roots of $\delta_{4,1}$ are $\sqrt{2} \omega_{24}^{5}, \sqrt{2} \omega_{24}^{11}$, $\sqrt{2} \omega_{24}^{13}, \sqrt{2} \omega_{24}^{19}$, with $\omega_{24}=e^{i \pi / 12}=((1+\sqrt{3})+(-1+\sqrt{3}) i) / 2 \sqrt{2}$. Observe that $F_{2}\left(n, t_{1}, t_{2}\right)$ can be obtained similarly, or by adding $F_{2}\left(n, t_{1}, t_{2}, 0\right)$ and $F_{2}\left(n, t_{1}, t_{2}, 1\right)$ as given in Theorem 6 . Likewise $F_{2}\left(n, t_{1}\right)$ can be obtained as $F_{2}\left(n, t_{1}, 0,0\right)+F_{2}\left(n, t_{1}, 0,1\right)+F_{2}\left(n, t_{1}, 1,0\right)+F_{2}\left(n, t_{1}, 1,1\right)$, and summing all the expressions gives $2^{n}$, as they must. 
4.1.2 Direct method. For odd $n$, applying Equations (32) to (34) we have

$$
\begin{aligned}
F_{2}\left(n, t_{1}, t_{2}, t_{3}\right)= & \#\left\{a \in \mathbb{F}_{2^{n}} \mid T_{1}(a)=t_{1}, T_{2}(a)=t_{2}, T_{3}(a)=t_{3}\right\} \\
= & \frac{1}{2} \#\left\{a_{0} \in \mathbb{F}_{2^{n}} \mid T_{2}\left(a_{0}^{2}+a_{0}+t_{1}\right)=t_{2}, T_{3}\left(a_{0}^{2}+a_{0}+t_{1}\right)=t_{3}\right\} \\
= & \frac{1}{2} \#\left\{a_{0} \in \mathbb{F}_{2^{n}} \mid T_{1}\left(a_{0}^{3}+a_{0}+t_{1}\left(\begin{array}{c}
n \\
2
\end{array}\right)\right)=t_{2}, T_{1}\left(a_{0}^{5}+a_{0}+t_{1}\left(a_{0}^{3}+a_{0}+\left(\begin{array}{c}
n \\
3
\end{array}\right)\right)\right)=t_{3}\right\} \\
= & \frac{1}{8} \#\left\{\left(a_{0}, a_{1}, a_{2}\right) \in\left(\mathbb{F}_{2^{n}}\right)^{3} \mid a_{1}^{2}+a_{1}=a_{0}^{3}+a_{0}+t_{1}\left(\begin{array}{c}
n \\
2
\end{array}\right)+t_{2},\right. \\
& \left.a_{2}^{2}+a_{2}=a_{0}^{5}+a_{0}+t_{1}\left(a_{0}^{3}+a_{0}+\left(\begin{array}{c}
n \\
3
\end{array}\right)\right)+t_{3}\right\} .
\end{aligned}
$$

These supersingular curves are all absolutely irreducible and are of genus 5 , and for $n$ odd their zeta functions reproduce Theorem 6 . Note that this method is slightly different to that used in [3, §5], since it counts the number of points on a curve defined by an intersection of two curves (with one variable in common), rather than the sum of the number of points on three curves. Letting $\delta_{2,2}=X^{2}+2$ (corresponding to the curve $C_{2,2} / \mathbb{F}_{2}: y^{2}+y=x^{3}+1$ ), further note that for all $n \geq 3$ we have

$$
\begin{aligned}
F_{2}(n, 0,0,0) & =\frac{1}{8} \#\left\{\left(a_{0}, a_{1}, a_{2}\right) \in\left(\mathbb{F}_{2^{n}}\right)^{3} \mid a_{1}^{2}+a_{1}=a_{0}^{3}+a_{0}, a_{2}^{2}+a_{2}=a_{0}^{5}+a_{0}\right\} \\
& =\frac{1}{8}\left(2^{n}-2 \rho_{n}\left(\delta_{2,1}\right)-\rho_{n}\left(\delta_{2,2}\right)-\rho_{n}\left(\delta_{4,1}\right)\right)
\end{aligned}
$$

since one does not need to parameterise any 'linear trace $=1$ ' conditions. Using the same basic observations from $[3, \S \S 4 \& 5]$ one can determine the formulae, valid for all $n \geq 3$, for all four $F_{2}\left(n, 0, t_{2}, t_{3}\right)$.

\subsection{Computing $F_{2}\left(n, t_{1}, t_{2}, t_{3}, t_{4}\right)$}

We begin by applying the indirect method.

4.2.1 Indirect method. Setting $\mathbf{f}=\left(T_{4}, T_{3}, T_{2}, T_{1}\right)$, by the transform $(24)$ we have

$\left[\begin{array}{l}F_{2}(n, 0,0,0,0) \\ F_{2}(n, 1,0,0,0) \\ F_{2}(n, 0,1,0,0) \\ F_{2}(n, 1,1,0,0) \\ F_{2}(n, 0,0,1,0) \\ F_{2}(n, 1,0,1,0) \\ F_{2}(n, 0,1,1,0) \\ F_{2}(n, 1,1,1,0) \\ F_{2}(n, 0,0,0,1) \\ F_{2}(n, 1,0,0,1) \\ F_{2}(n, 0,1,0,1) \\ F_{2}(n, 1,1,0,1) \\ F_{2}(n, 0,0,1,1) \\ F_{2}(n, 1,0,1,1) \\ F_{2}(n, 0,1,1,1) \\ F_{2}(n, 1,1,1,1)\end{array}\right]=\left[\begin{array}{l}N(\mathbf{0}) \\ N(\mathbf{1}) \\ N(\mathbf{2}) \\ N(\mathbf{3}) \\ N(\mathbf{4}) \\ N(\mathbf{5}) \\ N(\mathbf{6}) \\ N(\mathbf{7}) \\ N(\mathbf{8}) \\ N(\mathbf{9}) \\ N(\mathbf{1 0}) \\ N(\mathbf{1 1}) \\ N(\mathbf{1 2}) \\ N(\mathbf{1 3}) \\ N(\mathbf{1 4}) \\ N(\mathbf{1 5})\end{array}\right]=\frac{1}{8}\left[\begin{array}{rrrrrrrrrrrrrrrr}-7 & 1 & 1 & 1 & 1 & 1 & 1 & 1 & 1 & 1 & 1 & 1 & 1 & 1 & 1 & 1 \\ 1 & -1 & 1 & -1 & 1 & -1 & 1 & -1 & 1 & -1 & 1 & -1 & 1 & -1 & 1 & -1 \\ 1 & 1 & -1 & -1 & 1 & 1 & -1 & -1 & 1 & 1 & -1 & -1 & 1 & 1 & -1 & -1 \\ 1 & -1 & -1 & 1 & 1 & -1 & -1 & 1 & 1 & -1 & -1 & 1 & 1 & -1 & -1 & 1 \\ 1 & 1 & 1 & 1 & -1 & -1 & -1 & -1 & 1 & 1 & 1 & 1 & -1 & -1 & -1 & -1 \\ 1 & -1 & 1 & -1 & -1 & 1 & -1 & 1 & 1 & -1 & 1 & -1 & -1 & 1 & -1 & 1 \\ 1 & 1 & -1 & -1 & -1 & -1 & 1 & 1 & 1 & 1 & -1 & -1 & -1 & -1 & 1 & 1 \\ 1 & -1 & -1 & 1 & -1 & 1 & 1 & -1 & 1 & -1 & -1 & 1 & -1 & 1 & 1 & -1 \\ 1 & 1 & 1 & 1 & 1 & 1 & 1 & 1 & -1 & -1 & -1 & -1 & -1 & -1 & -1 & -1 \\ 1 & -1 & 1 & -1 & 1 & -1 & 1 & -1 & -1 & 1 & -1 & 1 & -1 & 1 & -1 & 1 \\ 1 & 1 & -1 & -1 & 1 & 1 & -1 & -1 & -1 & -1 & 1 & 1 & -1 & -1 & 1 & 1 \\ 1 & -1 & -1 & 1 & 1 & -1 & -1 & 1 & -1 & 1 & 1 & -1 & -1 & 1 & 1 & -1 \\ 1 & 1 & 1 & 1 & -1 & -1 & -1 & -1 & -1 & -1 & -1 & -1 & 1 & 1 & 1 & 1 \\ 1 \\ 1 & -1 & 1 & -1 & -1 & 1 & -1 & 1 & -1 & 1 & -1 & 1 & 1 & -1 & 1 & -1 \\ 1 & 1 & -1 & -1 & -1 & -1 & 1 & 1 & -1 & -1 & 1 & 1 & 1 & 1 & -1 & -1 \\ V(\mathbf{3} \cdot \mathbf{5} \cdot \mathbf{f}) \\ V(\mathbf{f} \cdot \mathbf{f}) \\ V(\mathbf{f} \cdot \mathbf{f}) \\ V(\mathbf{8} \cdot \mathbf{f}) \\ V(\mathbf{9} \cdot \mathbf{f}) \\ V(\mathbf{1 0} \cdot \mathbf{f}) \\ V(\mathbf{1 1} \cdot \mathbf{f}) \\ V(\mathbf{1 2} \cdot \mathbf{f}) \\ V(\mathbf{1 3} \cdot \mathbf{f}) \\ V(\mathbf{1 4} \cdot \mathbf{f}) \\ V(\mathbf{1 5} \cdot \mathbf{f})\end{array}\right]$

To determine $V(\mathbf{i} \cdot \mathbf{f})$ for $8 \leq i \leq 15$, we use Lemma 10 part (4). In particular, setting $\alpha=a_{0}^{2}$ and $\beta=a_{0}$ for $r_{0}=0$, and $\alpha=a_{0}^{2}+a_{0}$ and $\beta=1$ for $r_{0}=1$, and evaluating $\bmod 2$ gives the following:

$$
\begin{aligned}
T_{4}\left(a_{0}^{2}+a_{0}+r_{0}\right) & =T_{2}\left(a_{0}^{3}\right)+T_{2}\left(a_{0}\right)+T_{1}\left(a_{0}^{3}\right) T_{1}\left(a_{0}\right) \\
& +T_{1}\left(a_{0}^{7}+a_{0}^{5}+a_{0}^{3}+r_{0}\left(a_{0}^{3}+a_{0}+\left(a_{0}^{3}+a_{0}\right)\left(\begin{array}{l}
n \\
2
\end{array}\right)+\left(\begin{array}{l}
n \\
4
\end{array}\right)\right)\right) .
\end{aligned}
$$


This can be linearised using the substitutions $a_{0}=a_{1}^{2}+a_{1}+r_{1}$ and $a_{0}^{3}=a_{2}^{2}+a_{2}+r_{2}$, where $r_{1}, r_{2} \in \mathbb{F}_{2}$ are the traces of $a_{0}$ and $a_{0}^{3}$ respectively. This results in ${ }^{\dagger}$

$$
\begin{aligned}
T_{4}\left(a_{0}^{2}+a_{0}+r_{0}\right) & =T_{1}\left(a_{2}^{3}+a_{2}+a_{1}^{3}+a_{1}+a_{0}^{7}+a_{0}^{5}+r_{0} r_{1}+r_{0} r_{2}+r_{1} r_{2}+r_{2}\right. \\
& \left.+\left(r_{0}+1\right)\left(r_{1}+r_{2}\right)\left(\begin{array}{l}
n \\
2
\end{array}\right)+r_{0}\left(\begin{array}{l}
n \\
4
\end{array}\right)\right) .
\end{aligned}
$$

For $8 \leq i \leq 15$ let $\mathbf{i}=\left(i_{3}, i_{2}, i_{1}, i_{0}\right)$. The curves we are interested in are given by the following intersections:

$$
\begin{aligned}
a_{3}^{2}+a_{3} & =i_{3}\left(a_{2}^{3}+a_{2}+a_{1}^{3}+a_{1}+a_{0}^{7}+a_{0}^{5}+r_{0} r_{1}+r_{0} r_{2}+r_{1} r_{2}+r_{2}+\left(r_{0}+1\right)\left(r_{1}+r_{2}\right)\left(\begin{array}{c}
n \\
2
\end{array}\right)+r_{0}\left(\begin{array}{c}
n \\
4
\end{array}\right)\right) \\
& +i_{2}\left(a_{0}^{5}+a_{0}+r_{0}\left(a_{0}^{3}+a_{0}+\left(\begin{array}{c}
n \\
3
\end{array}\right)\right)\right)+i_{1}\left(a_{0}^{3}+a_{0}+r_{0}\left(\begin{array}{c}
n \\
2
\end{array}\right)\right)+i_{0} r_{0} \\
a_{0} & =a_{1}^{2}+a_{1}+r_{1} \\
a_{0}^{3} & =a_{2}^{2}+a_{2}+r_{2} .
\end{aligned}
$$

For $i_{3}=1$ the genus of all of these absolutely irreducible curves is 14 . Corollary 1 implies that mod 2 one has

$$
\left(\left(\begin{array}{l}
n \\
4
\end{array}\right),\left(\begin{array}{l}
n \\
3
\end{array}\right),\left(\begin{array}{l}
n \\
2
\end{array}\right),\left(\begin{array}{l}
n \\
1
\end{array}\right)\right) \equiv\left\{\begin{array}{lll}
(0,0,0,1) & \text { if } n \equiv 1 & (\bmod 8) \\
(0,1,1,1) & \text { if } n \equiv 3 & (\bmod 8) \\
(1,0,0,1) & \text { if } n \equiv 5 & (\bmod 8) \\
(1,1,1,1) & \text { if } n \equiv 7 & (\bmod 8)
\end{array}\right.
$$

and hence there are four cases to consider when computing the zeta functions of each of the curves (38). In order to express $F_{2}\left(n, t_{1}, t_{2}, t_{3}, t_{4}\right)$ compactly, we further define the following polynomials:

$$
\begin{aligned}
& \delta_{8,1}=X^{8}+4 X^{7}+6 X^{6}+4 X^{5}+2 X^{4}+8 X^{3}+24 X^{2}+32 X+16, \\
& \delta_{8,2}=X^{8}+2 X^{6}+4 X^{5}+2 X^{4}+8 X^{3}+8 X^{2}+16 .
\end{aligned}
$$

Note that by Theorem 5, neither $\delta_{8,1}$ or $\delta_{8,2}$ are the characteristic polynomials of the Frobenius endomorphism of supersingular abelian varieties. There are two other polynomials which occur as factors of the characteristic polynomial of Frobenius of the above curves, but they are even polynomials and hence can be ignored for $n$ odd. Using Magma to compute the zeta functions of the curves (38) and applying (36) gives the following result.

${ }^{\dagger}$ See NewtonApproach_1_le_7.mw to verify the linearised expressions for 4,5,6 and 7 coefficients. 
Theorem 7. For $n \geq 4$ we have

$F_{2}(n, 0,0,0,0)=2^{n-4}-\frac{1}{16}\left(4 \rho_{n}\left(\delta_{2,1}\right)+\rho_{n}\left(\delta_{4,1}\right)+\rho_{n}\left(\delta_{8,1}\right)+\rho_{n}\left(\delta_{8,2}\right)\right) \quad$ if $n \equiv 1,3,5,7 \quad(\bmod 8)$

$F_{2}(n, 1,0,0,0)=2^{n-4}-\frac{1}{16} \cdot\left\{\begin{array}{lll}4 \rho_{n}\left(\delta_{2,1}\right)+\rho_{n}\left(\delta_{4,1}\right)+\rho_{n}\left(\delta_{8,1}\right)+\rho_{n}\left(\delta_{8,2}\right) & \text { if } n \equiv 1 \quad(\bmod 8) \\ 2 \rho_{n}\left(\delta_{2,1}\right)-\rho_{n}\left(\delta_{4,1}\right)-\rho_{n}\left(\delta_{8,1}\right)+\rho_{n}\left(\delta_{8,2}\right) & \text { if } n \equiv 3 \quad(\bmod 8) \\ \rho_{n}\left(\delta_{4,1}\right)-\rho_{n}\left(\delta_{8,1}\right)-\rho_{n}\left(\delta_{8,2}\right) & \text { if } n \equiv 5 \quad(\bmod 8) \\ -2 \rho_{n}\left(\delta_{2,1}\right)-\rho_{n}\left(\delta_{4,1}\right)+\rho_{n}\left(\delta_{8,1}\right)-\rho_{n}\left(\delta_{8,2}\right) & \text { if } n \equiv 7 \quad(\bmod 8)\end{array}\right.$

$F_{2}(n, 0,1,0,0)=2^{n-4}-\frac{1}{16} \cdot\left\{\begin{array}{lll}-2 \rho_{n}\left(\delta_{2,1}\right)-\rho_{n}\left(\delta_{4,1}\right)+\rho_{n}\left(\delta_{8,1}\right)-\rho_{n}\left(\delta_{8,2}\right) & \text { if } n \equiv 1,5 & (\bmod 8) \\ 2 \rho_{n}\left(\delta_{2,1}\right)-\rho_{n}\left(\delta_{4,1}\right)-\rho_{n}\left(\delta_{8,1}\right)+\rho_{n}\left(\delta_{8,2}\right) & \text { if } n \equiv 3,7 & (\bmod 8)\end{array}\right.$

$F_{2}(n, 1,1,0,0)=2^{n-4}-\frac{1}{16} \cdot\left\{\begin{array}{lll}-4 \rho_{n}\left(\delta_{2,1}\right)+\rho_{n}\left(\delta_{4,1}\right)+\rho_{n}\left(\delta_{8,1}\right)+\rho_{n}\left(\delta_{8,2}\right) & \text { if } n \equiv 1 \quad(\bmod 8) \\ -2 \rho_{n}\left(\delta_{2,1}\right)-\rho_{n}\left(\delta_{4,1}\right)+\rho_{n}\left(\delta_{8,1}\right)-\rho_{n}\left(\delta_{8,2}\right) & \text { if } n \equiv 3 \quad(\bmod 8) \\ \rho_{n}\left(\delta_{4,1}\right)-\rho_{n}\left(\delta_{8,1}\right)-\rho_{n}\left(\delta_{8,2}\right) & \text { if } n \equiv 5 \quad(\bmod 8) \\ 2 \rho_{n}\left(\delta_{2,1}\right)-\rho_{n}\left(\delta_{4,1}\right)-\rho_{n}\left(\delta_{8,1}\right)+\rho_{n}\left(\delta_{8,2}\right) & \text { if } n \equiv 7 \quad(\bmod 8)\end{array}\right.$

$F_{2}(n, 0,0,1,0)=2^{n-4}-\frac{1}{16}\left(-2 \rho_{n}\left(\delta_{2,1}\right)-\rho_{n}\left(\delta_{4,1}\right)+\rho_{n}\left(\delta_{8,1}\right)-\rho_{n}\left(\delta_{8,2}\right)\right)$ if $n \equiv 1,3,5,7 \quad(\bmod 8)$

$F_{2}(n, 1,0,1,0)=2^{n-4}-\frac{1}{16} \cdot\left\{\begin{array}{lll}-2 \rho_{n}\left(\delta_{2,1}\right)-\rho_{n}\left(\delta_{4,1}\right)+\rho_{n}\left(\delta_{8,1}\right)-\rho_{n}\left(\delta_{8,2}\right) & \text { if } n \equiv 1 \quad(\bmod 8) \\ -4 \rho_{n}\left(\delta_{2,1}\right)+\rho_{n}\left(\delta_{4,1}\right)+\rho_{n}\left(\delta_{8,1}\right)+\rho_{n}\left(\delta_{8,2}\right) & \text { if } n \equiv 3 \quad(\bmod 8) \\ 2 \rho_{n}\left(\delta_{2,1}\right)-\rho_{n}\left(\delta_{4,1}\right)-\rho_{n}\left(\delta_{8,1}\right)+\rho_{n}\left(\delta_{8,2}\right) & \text { if } n \equiv 5 \quad(\bmod 8) \\ \rho_{n}\left(\delta_{4,1}\right)-\rho_{n}\left(\delta_{8,1}\right)-\rho_{n}\left(\delta_{8,2}\right) & \text { if } n \equiv 7 & (\bmod 8)\end{array}\right.$

$F_{2}(n, 0,1,1,0)=2^{n-4}-\frac{1}{16} \cdot \begin{cases}\rho_{n}\left(\delta_{4,1}\right)-\rho_{n}\left(\delta_{8,1}\right)-\rho_{n}\left(\delta_{8,2}\right) & \text { if } n \equiv 1,5 \quad(\bmod 8) \\ -4 \rho_{n}\left(\delta_{2,1}\right)+\rho_{n}\left(\delta_{4}\right)+\rho_{n}\left(\delta_{8,1}\right)+\rho_{n}\left(\delta_{8,2}\right) & \text { if } n 3,7 \quad(\bmod 8)\end{cases}$

$F_{2}(n, 1,1,1,0)=2^{n-4}-\frac{1}{16} \cdot\left\{\begin{array}{lll}2 \rho_{n}\left(\delta_{2,1}\right)-\rho_{n}\left(\delta_{4,1}\right)-\rho_{n}\left(\delta_{8,1}\right)+\rho_{n}\left(\delta_{8,2}\right) & \text { if } n \equiv 1 \quad(\bmod 8) \\ 4 \rho_{n}\left(\delta_{2,1}\right)+\rho_{n}\left(\delta_{4,1}\right)+\rho_{n}\left(\delta_{8,1}\right)+\rho_{n}\left(\delta_{8,2}\right) & \text { if } n \equiv 3 \quad(\bmod 8) \\ -2 \rho_{n}\left(\delta_{2,1}\right)-\rho_{n}\left(\delta_{4,1}\right)+\rho_{n}\left(\delta_{8,1}\right)-\rho_{n}\left(\delta_{8,2}\right) & \text { if } n \equiv 5 & (\bmod 8) \\ \rho_{n}\left(\delta_{4,1}\right)-\rho_{n}\left(\delta_{8,1}\right)-\rho_{n}\left(\delta_{8,2}\right) & \text { if } n \equiv 7 & (\bmod 8)\end{array}\right.$

$F_{2}(n, 0,0,0,1)=2^{n-4}-\frac{1}{16}\left(\rho_{n}\left(\delta_{4,1}\right)-\rho_{n}\left(\delta_{8,1}\right)-\rho_{n}\left(\delta_{8,2}\right)\right)$ if $n \equiv 1,3,5,7 \quad(\bmod 8)$

$F_{2}(n, 1,0,0,1)=2^{n-4}-\frac{1}{16} \cdot\left\{\begin{array}{lll}\rho_{n}\left(\delta_{4,1}\right)-\rho_{n}\left(\delta_{8,1}\right)-\rho_{n}\left(\delta_{8,2}\right) & \text { if } n \equiv 1 \quad(\bmod 8) \\ -2 \rho_{n}\left(\delta_{2,1}\right)-\rho_{n}\left(\delta_{4,1}\right)+\rho_{n}\left(\delta_{8,1}\right)-\rho_{n}\left(\delta_{8,2}\right) & \text { if } n \equiv 3 \quad(\bmod 8) \\ 4 \rho_{n}\left(\delta_{2,1}\right)+\rho_{n}\left(\delta_{4,1}\right)+\rho_{n}\left(\delta_{8,1}\right)+\rho_{n}\left(\delta_{8,2}\right) & \text { if } n \equiv 5 \quad(\bmod 8) \\ 2 \rho_{n}\left(\delta_{2,1}\right)-\rho_{n}\left(\delta_{4,1}\right)-\rho_{n}\left(\delta_{8,1}\right)+\rho_{n}\left(\delta_{8,2}\right) & \text { if } n \equiv 7 & (\bmod 8)\end{array}\right.$

$F_{2}(n, 0,1,0,1)=2^{n-4}-\frac{1}{16} \cdot\left\{\begin{array}{lll}2 \rho_{n}\left(\delta_{2,1}\right)-\rho_{n}\left(\delta_{4,1}\right)-\rho_{n}\left(\delta_{8,1}\right)+\rho_{n}\left(\delta_{8,2}\right) & \text { if } n \equiv 1,5 \quad(\bmod 8) \\ -2 \rho_{n}\left(\delta_{2,1}\right)-\rho_{n}\left(\delta_{4,1}\right)+\rho_{n}\left(\delta_{8,1}\right)-\rho_{n}\left(\delta_{8,2}\right) & \text { if } n \equiv 3,7 & (\bmod 8)\end{array}\right.$

$F_{2}(n, 1,1,0,1)=2^{n-4}-\frac{1}{16} \cdot\left\{\begin{array}{lll}\rho_{n}\left(\delta_{4,1}\right)-\rho_{n}\left(\delta_{8,1}\right)-\rho_{n}\left(\delta_{8,2}\right) & \text { if } n \equiv 1 \quad(\bmod 8) \\ 2 \rho_{n}\left(\delta_{2,1}\right)-\rho_{n}\left(\delta_{4,1}\right)-\rho_{n}\left(\delta_{8,1}\right)+\rho_{n}\left(\delta_{8,2}\right) & \text { if } n \equiv 3 \quad(\bmod 8) \\ -4 \rho_{n}\left(\delta_{2,1}\right)+\rho_{n}\left(\delta_{4,1}\right)+\rho_{n}\left(\delta_{8,1}\right)+\rho_{n}\left(\delta_{8,2}\right) & \text { if } n \equiv 5 \quad(\bmod 8) \\ -2 \rho_{n}\left(\delta_{2,1}\right)-\rho_{n}\left(\delta_{4,1}\right)+\rho_{n}\left(\delta_{8,1}\right)-\rho_{n}\left(\delta_{8,2}\right) & \text { if } n \equiv 7 & (\bmod 8)\end{array}\right.$

$F_{2}(n, 0,0,1,1)=2^{n-4}-\frac{1}{16}\left(2 \rho_{n}\left(\delta_{2,1}\right)-\rho_{n}\left(\delta_{4,1}\right)-\rho_{n}\left(\delta_{8,1}\right)+\rho_{n}\left(\delta_{8,2}\right)\right) \quad$ if $n \equiv 1,3,5,7 \quad(\bmod 8)$

$F_{2}(n, 1,0,1,1)=2^{n-4}-\frac{1}{16} \cdot\left\{\begin{array}{lll}2 \rho_{n}\left(\delta_{2,1}\right)-\rho_{n}\left(\delta_{4,1}\right)-\rho_{n}\left(\delta_{8,1}\right)+\rho_{n}\left(\delta_{8,2}\right) & \text { if } n \equiv 1 \quad(\bmod 8) \\ \rho_{n}\left(\delta_{4,1}\right)-\rho_{n}\left(\delta_{8,1}\right)-\rho_{n}\left(\delta_{8,2}\right) & \text { if } n \equiv 3 \quad(\bmod 8) \\ -2 \rho_{n}\left(\delta_{2,1}\right)-\rho_{n}\left(\delta_{4,1}\right)+\rho_{n}\left(\delta_{8,1}\right)-\rho_{n}\left(\delta_{8,2}\right) & \text { if } n \equiv 5 \quad(\bmod 8) \\ -4 \rho_{n}\left(\delta_{2,1}\right)+\rho_{n}\left(\delta_{4,1}\right)+\rho_{n}\left(\delta_{8,1}\right)+\rho_{n}\left(\delta_{8,2}\right) & \text { if } n \equiv 7 \quad(\bmod 8)\end{array}\right.$

$F_{2}(n, 0,1,1,1)=2^{n-4}-\frac{1}{16} \cdot\left\{\begin{array}{lll}-4 \rho_{n}\left(\delta_{2,1}\right)+\rho_{n}\left(\delta_{4,1}\right)+\rho_{n}\left(\delta_{8,1}\right)+\rho_{n}\left(\delta_{8,2}\right) & \text { if } n \equiv 1,5 \quad(\bmod 8) \\ \rho_{n}\left(\delta_{4,1}\right)-\rho_{n}\left(\delta_{8,1}\right)-\rho_{n}\left(\delta_{8,2}\right) & \text { if } n \equiv 3,7 & (\bmod 8)\end{array}\right.$

$F_{2}(n, 1,1,1,1)=2^{n-4}-\frac{1}{16} \cdot\left\{\begin{array}{lll}-2 \rho_{n}\left(\delta_{2,1}\right)-\rho_{n}\left(\delta_{4,1}\right)+\rho_{n}\left(\delta_{8,1}\right)-\rho_{n}\left(\delta_{8,2}\right) & \text { if } n \equiv 1 \quad(\bmod 8) \\ \rho_{n}\left(\delta_{4,1}\right)-\rho_{n}\left(\delta_{8,1}\right)-\rho_{n}\left(\delta_{8,2}\right) & \text { if } n \equiv 3 \quad(\bmod 8) \\ 2 \rho_{n}\left(\delta_{2,1}\right)-\rho_{n}\left(\delta_{4,1}\right)-\rho_{n}\left(\delta_{8,1}\right)+\rho_{n}\left(\delta_{8,2}\right) & \text { if } n \equiv 5 \quad(\bmod 8) \\ 4 \rho_{n}\left(\delta_{2,1}\right)+\rho_{n}\left(\delta_{4,1}\right)+\rho_{n}\left(\delta_{8,1}\right)+\rho_{n}\left(\delta_{8,2}\right) & \text { if } n \equiv 7 & (\bmod 8)\end{array}\right.$ 
One can check that the roots of $\delta_{8,1}$ are $\alpha_{1}, \alpha_{2}, \alpha_{3}, \alpha_{4}$ and their complex conjugates $\overline{\alpha_{1}}, \overline{\alpha_{2}}, \overline{\alpha_{3}}, \overline{\alpha_{4}}$, where:

$$
\begin{aligned}
& \alpha_{1}=-\frac{1}{2}+\frac{\sqrt{2}}{4}(1+\sqrt{7+4 \sqrt{2}})-\frac{\sqrt{2}}{4}(1+\sqrt{5-2 \sqrt{2}}) i \\
& \alpha_{2}=-\frac{1}{2}+\frac{\sqrt{2}}{4}(1-\sqrt{7+4 \sqrt{2}})-\frac{\sqrt{2}}{4}(1-\sqrt{5-2 \sqrt{2}}) i \\
& \alpha_{3}=-\frac{1}{2}-\frac{\sqrt{2}}{4}\left(1+\frac{1}{\sqrt{17}}(3 \sqrt{2}-1) \sqrt{5-2 \sqrt{2}}\right)+\frac{\sqrt{2}}{4}\left(1-\frac{1}{\sqrt{17}}(3 \sqrt{2}-1) \sqrt{7+4 \sqrt{2}}\right) i \\
& \alpha_{4}=-\frac{1}{2}-\frac{\sqrt{2}}{4}\left(1-\frac{1}{\sqrt{17}}(3 \sqrt{2}-1) \sqrt{5-2 \sqrt{2}}\right)+\frac{\sqrt{2}}{4}\left(1+\frac{1}{\sqrt{17}}(3 \sqrt{2}-1) \sqrt{7+4 \sqrt{2}}\right) i .
\end{aligned}
$$

One can also check that the roots of $\delta_{8,2}$ are $i \alpha_{1}, i \alpha_{2}, i \alpha_{3}, i \alpha_{4}$ and $\overline{i \alpha_{1}}, \overline{i \alpha_{2}}, \overline{i \alpha_{3}}, \overline{i \alpha_{4}}$. In Theorem 7 the formulae for each $F_{2}\left(n, t_{1}, t_{2}, t_{3}, t_{4}\right)$ and each odd $n \bmod 8$ have non-supersingular terms of the form $\pm \rho_{n}\left(\delta_{8,1}\right) \pm \rho_{n}\left(\delta_{8,2}\right)$ or $\pm \rho_{n}\left(\delta_{8,1}\right) \mp \rho_{n}\left(\delta_{8,2}\right)$. A simple application of Kronecker's theorem to the phases of these non-supersingular Weil numbers allows one to deduce that the formulae are not periodic in $n$. We leave stating what abelian varieties $\delta_{8,1}$ and $\delta_{8,2}$ are the characteristic polynomials of Frobenius for as an open problem, but note that they must be isogenous over $\mathbb{F}_{2^{4}}$.

An alternative parameterisation. We now present an alternative parameterisation of Eq. (37) which requires one less variable to linearise. In particular, we have

$$
\begin{aligned}
T_{4}\left(a_{0}^{2}+a_{0}+r_{0}\right) & =T_{2}\left(a_{0}^{3}\right)+T_{2}\left(a_{0}\right)+T_{1}\left(a_{0}^{3}\right) T_{1}\left(a_{0}\right) \\
& +T_{1}\left(a_{0}^{7}+a_{0}^{5}+a_{0}^{3}+r_{0}\left(a_{0}^{3}+a_{0}+\left(a_{0}^{3}+a_{0}\right)\left(\begin{array}{l}
n \\
2
\end{array}\right)+\left(\begin{array}{l}
n \\
4
\end{array}\right)\right)\right) \\
& =T_{2}\left(a_{0}^{3}+a_{0}\right)+T_{1}\left(a_{0}^{7}+a_{0}^{5}+a_{0}^{3}+a_{0}+r_{0}\left(a_{0}^{3}+a_{0}+\left(a_{0}^{3}+a_{0}\right)\left(\begin{array}{c}
n \\
2
\end{array}\right)+\left(\begin{array}{c}
n \\
4
\end{array}\right)\right)\right),
\end{aligned}
$$

where the second equality follows from Lemma 10 part (2), by setting $\alpha=a_{0}^{3}$ and $\beta=a_{0}$. This can be linearised using the substitution $a_{0}^{3}+a_{0}=a_{1}^{2}+a_{1}+r_{1}$, where $r_{1}$ is the trace of $a_{0}^{3}+a_{0}$. This results in

$$
\begin{aligned}
T_{4}\left(a_{0}^{2}+a_{0}+r_{0}\right) & =T_{1}\left(a_{1}^{3}+a_{1}+a_{0}^{7}+a_{0}^{5}+a_{0}^{3}+a_{0}\right. \\
& \left.+r_{0}\left(a_{0}^{3}+a_{0}+\left(a_{0}^{3}+a_{0}\right)\left(\begin{array}{l}
n \\
2
\end{array}\right)+\left(\begin{array}{l}
n \\
4
\end{array}\right)\right)+r_{1}\left(\begin{array}{l}
n \\
2
\end{array}\right)\right) .
\end{aligned}
$$

For $8 \leq i \leq 15$ let $\mathbf{i}=\left(i_{3}, i_{2}, i_{1}, i_{0}\right)$. The curves we are interested in are given by the following intersections:

$$
\begin{aligned}
a_{2}^{2}+a_{2} & =i_{3}\left(a_{1}^{3}+a_{1}+a_{0}^{7}+a_{0}^{5}+a_{0}^{3}+a_{0}+r_{0}\left(a_{0}^{3}+a_{0}+\left(a_{0}^{3}+a_{0}\right)\left(\begin{array}{l}
n \\
2
\end{array}\right)+\left(\begin{array}{l}
n \\
4
\end{array}\right)\right)+r_{1}\left(\begin{array}{l}
n \\
2
\end{array}\right)\right) \\
& +i_{2}\left(a_{0}^{5}+a_{0}+r_{0}\left(a_{0}^{3}+a_{0}+\left(\begin{array}{l}
n \\
3
\end{array}\right)\right)\right)+i_{1}\left(a_{0}^{3}+a_{0}+r_{0}\left(\begin{array}{l}
n \\
2
\end{array}\right)\right)+i_{0} r_{0} \\
a_{0}^{3}+a_{0} & =a_{1}^{2}+a_{1}+r_{1} .
\end{aligned}
$$

For $i_{3}=1$ the genus of all of these absolutely irreducible curves is 7 - rather than 14 as in the first parameterisation - and therefore the characteristic polynomials of Frobenius have lower degrees than before. Indeed, there are fewer even polynomials appearing as factors and those that are not even occur to lower powers. Nevertheless, once (36) is applied one again obtains the formulae given in Theorem 7 . 
4.2.2 Direct method. For odd $n$, applying Equations (32) to (34) and (39) we have

$$
\begin{aligned}
F_{2}\left(n, t_{1}, t_{2}, t_{3}, t_{4}\right)= & \#\left\{a \in \mathbb{F}_{2^{n}} \mid T_{1}(a)=t_{1}, T_{2}(a)=t_{2}, T_{3}(a)=t_{3}, T_{4}(a)=t_{4}\right\} \\
= & \frac{1}{2} \#\left\{a_{0} \in \mathbb{F}_{2^{n}} \mid T_{2}\left(a_{0}^{2}+a_{0}+t_{1}\right)=t_{2}, T_{3}\left(a_{0}^{2}+a_{0}+t_{1}\right)=t_{3}, T_{4}\left(a_{0}^{2}+a_{0}+t_{1}\right)=t_{4}\right\} \\
= & \frac{1}{2} \#\left\{a_{0} \in \mathbb{F}_{2^{n}} \mid T_{1}\left(a_{0}^{3}+a_{0}+t_{1}\left(\begin{array}{c}
n \\
2
\end{array}\right)\right)=t_{2}, T_{1}\left(a_{0}^{5}+a_{0}+t_{1}\left(a_{0}^{3}+a_{0}+\left(\begin{array}{c}
n \\
3
\end{array}\right)\right)\right)=t_{3},\right. \\
& \left.T_{2}\left(a_{0}^{3}+a_{0}\right)+T_{1}\left(a_{0}^{7}+a_{0}^{5}+a_{0}^{3}+a_{0}+t_{1}\left(a_{0}^{3}+a_{0}+\left(a_{0}^{3}+a_{0}\right)\left(\begin{array}{c}
n \\
2
\end{array}\right)+\left(\begin{array}{c}
n \\
4
\end{array}\right)\right)\right)=t_{4}\right\} \\
= & \frac{1}{16} \#\left\{\left(a_{0}, a_{1}, a_{2}, a_{3}\right) \in\left(\mathbb{F}_{2^{n}}\right)^{4} \mid a_{1}^{2}+a_{1}=a_{0}^{3}+a_{0}+t_{1}\left(\begin{array}{c}
n \\
2
\end{array}\right)+t_{2},\right. \\
& a_{2}^{2}+a_{2}=a_{0}^{5}+a_{0}+t_{1}\left(a_{0}^{3}+a_{0}+\left(\begin{array}{c}
n \\
3
\end{array}\right)\right)+t_{3}, a_{3}^{2}+a_{3}=a_{1}^{3}+a_{1}+\left(t_{1}+t_{2}\right)\left(\begin{array}{c}
n \\
2
\end{array}\right)+ \\
& \left.a_{0}^{7}+a_{0}^{5}+a_{0}^{3}+a_{0}+t_{1}\left(a_{0}^{3}+a_{0}+\left(a_{0}^{3}+a_{0}\right)\left(\begin{array}{c}
n \\
2
\end{array}\right)+\left(\begin{array}{c}
n \\
4
\end{array}\right)\right)+t_{4}\right\},
\end{aligned}
$$

where we have used Lemma 10 part (2) and the parameterisation of $T_{2}(a)=t_{2}$ to compute $T_{2}\left(a_{0}^{3}+a_{0}\right)=$ $T_{2}\left(a_{1}^{2}+a_{1}+t_{1}\left(\begin{array}{c}n \\ 2\end{array}\right)+t_{2}\right)=T_{2}\left(a_{1}^{2}+a_{1}\right)+T_{2}\left(t_{1}\left(\begin{array}{l}n \\ 2\end{array}\right)+t_{2}\right)+T_{1}\left(a_{1}^{2}+a_{1}\right) T_{1}\left(t_{1}\left(\begin{array}{c}n \\ 2\end{array}\right)+t_{2}\right)+T_{1}\left(\left(t_{1}\left(\begin{array}{c}n \\ 2\end{array}\right)+t_{2}\right)\left(a_{1}^{2}+a_{1}\right)\right)=$ $T_{1}\left(a_{1}^{3}+a_{1}\right)+\left(t_{1}+t_{2}\right)\left(\begin{array}{l}n \\ 2\end{array}\right)$, since the last two terms are zero. These curves are all absolutely irreducible and of genus 17, and for $n$ odd their zeta functions reproduce Theorem 7 .

4.2.3 General formulae. We have the following result.

Theorem 8. For $n \geq 4$ we have

$$
\begin{aligned}
& F_{2}(n, 0,0,0,0)=2^{n-4}-\frac{1}{16}\left(4 \rho_{n}\left(\delta_{2,1}\right)+3 \rho_{n}\left(\delta_{2,2}\right)+\rho_{n}\left(\delta_{4,1}\right)+\rho_{n}\left(\delta_{8,1}\right)+\rho_{n}\left(\delta_{8,2}\right)\right), \\
& F_{2}(n, 0,0,0,1)=2^{n-4}-\frac{1}{16}\left(-\rho_{n}\left(\delta_{2,2}\right)+\rho_{n}\left(\delta_{4,1}\right)-\rho_{n}\left(\delta_{8,1}\right)-\rho_{n}\left(\delta_{8,2}\right)\right), \\
& F_{2}(n, 0,0,1,0)=2^{n-4}-\frac{1}{16}\left(-2 \rho_{n}\left(\delta_{2,1}\right)+\rho_{n}\left(\delta_{2,2}\right)-\rho_{n}\left(\delta_{4,1}\right)+\rho_{n}\left(\delta_{8,1}\right)-\rho_{n}\left(\delta_{8,2}\right)\right), \\
& F_{2}(n, 0,0,1,1)=2^{n-4}-\frac{1}{16}\left(2 \rho_{n}\left(\delta_{2,1}\right)-3 \rho_{n}\left(\delta_{2,2}\right)-\rho_{n}\left(\delta_{4,1}\right)-\rho_{n}\left(\delta_{8,1}\right)+\rho_{n}\left(\delta_{8,2}\right)\right) .
\end{aligned}
$$

Proof. Using the direct method, for all $n \geq 4$ we have

$$
\begin{aligned}
F_{2}(n, 0,0,0,0)= & \frac{1}{16} \#\left\{\left(a_{0}, a_{1}, a_{2}, a_{3}\right) \in\left(\mathbb{F}_{2^{n}}\right)^{4} \mid a_{1}^{2}+a_{1}=a_{0}^{3}+a_{0}, a_{2}^{2}+a_{2}=a_{0}^{5}+a_{0},\right. \\
& \left.a_{3}^{2}+a_{3}=a_{1}^{3}+a_{1}+a_{0}^{7}+a_{0}^{5}+a_{0}^{3}+a_{0}\right\} \\
= & 2^{n-4}-\frac{1}{16}\left(4 \rho_{n}\left(\delta_{2,1}\right)+3 \rho_{n}\left(\delta_{2,2}\right)+\rho_{n}\left(\delta_{4,1}\right)+\rho_{n}\left(\delta_{8,1}\right)+\rho_{n}\left(\delta_{8,2}\right)\right),
\end{aligned}
$$

since one does not need to parameterise any 'linear trace $=1$ ' conditions. Since $F_{2}(n, 0,0,0)=F_{2}(n, 0,0,0,0)+$ $F_{2}(n, 0,0,0,1)$, for all $n \geq 4$ we also have:

$$
F_{2}(n, 0,0,0,1)=2^{n-4}-\frac{1}{16}\left(-\rho_{n}\left(\delta_{2,2}\right)+\rho_{n}\left(\delta_{4,1}\right)-\rho_{n}\left(\delta_{8,1}\right)-\rho_{n}\left(\delta_{8,2}\right)\right) .
$$

Omitting the $T_{3}(a)$ condition, we obtain:

$$
\begin{aligned}
F_{2}(n, 0,0, *, 0) & =\frac{1}{8} \#\left\{\left(a_{0}, a_{1}, a_{2}\right) \in\left(\mathbb{F}_{2^{n}}\right)^{3} \mid a_{1}^{2}+a_{1}=a_{0}^{3}+a_{0}, a_{2}^{2}+a_{2}=a_{1}^{3}+a_{1}+a_{0}^{7}+a_{0}^{5}+a_{0}^{3}+a_{0}\right\} \\
& =2^{n-3}-\frac{1}{8}\left(\rho_{n}\left(\delta_{2,1}\right)+2 \rho_{n}\left(\delta_{2,2}\right)+\rho_{n}\left(\delta_{8,1}\right)\right)
\end{aligned}
$$


this intersection describing an absolutely irreducible curve of genus 7 . Since $F_{2}(n, 0,0, *, 0)=F_{2}(n, 0,0,0,0)+$ $F_{2}(n, 0,0,1,0)$ we also have:

$$
F_{2}(n, 0,0,1,0)=2^{n-4}-\frac{1}{16}\left(-2 \rho_{n}\left(\delta_{2,1}\right)+\rho_{n}\left(\delta_{2,2}\right)-\rho_{n}\left(\delta_{4,1}\right)+\rho_{n}\left(\delta_{8,1}\right)-\rho_{n}\left(\delta_{8,2}\right)\right) .
$$

Moreover, since $F_{2}(n, 0,0)=F_{2}(n, 0,0,0,0)+F_{2}(n, 0,0,0,1)+F_{2}(n, 0,0,1,0)+F_{2}(n, 0,0,1,1)$ we have

$$
F_{2}(n, 0,0,1,1)=2^{n-4}-\frac{1}{16}\left(2 \rho_{n}\left(\delta_{2,1}\right)-3 \rho_{n}\left(\delta_{2,2}\right)-\rho_{n}\left(\delta_{4,1}\right)-\rho_{n}\left(\delta_{8,1}\right)+\rho_{n}\left(\delta_{8,2}\right)\right) .
$$

Note that only the $F_{2}(n, 0,0,0,0)$ formula comes from the characteristic polynomial of Frobenius of a curve, since the other three have terms with the wrong sign. Note also that the total degree of the corresponding polynomials (numerator degree plus denominator degree) is $2 \cdot 17=34$ only for $F_{2}(n, 0,0,0,0)$. This does not contradict the fact that the direct method always produces curves of genus 17 , since the direct method in general only represents $F_{2}\left(n, t_{1}, t_{2}, t_{3}, t_{4}\right)$ for $n$ odd, so there are cancellations, and furthermore all the featured ' + ' signs in the formulae for $F_{2}\left(n, 0,0, t_{3}, t_{4}\right)$ may be replaced by '-' signs once the featured polynomials $\delta(X)$ are replaced with $\delta(-X)$.

If one similarly tries to omit the $T_{2}(a)$ condition then one can not automatically simplify the $T_{2}\left(a_{0}^{3}+a_{0}\right)$ term which arises from the condition $T_{4}\left(a_{0}^{2}+a_{0}\right)=0$; one is forced to condition on whether the linear trace of $a_{0}^{3}+a_{0}$ is 0 or 1 , in which case one needs $n$ to be odd in order to parameterise the latter condition. Therefore, it is apparently not possible to find formulae for all $n \geq 4$ for $F_{2}\left(n, 0,1, t_{3}, t_{4}\right)$ with this approach. Nevertheless, we expect that similar formulae hold for all $n \geq 4$ for each $F_{2}\left(n, t_{1}, t_{2}, t_{3}, t_{4}\right)$, with additional terms arising from the $n$-th powers of roots of a set of even polynomials. Furthermore, if for a given $F_{2}\left(n, t_{1}, t_{2}, t_{3}, t_{4}\right)$ the coefficients of the various $\rho_{n}\left(\delta_{i}\right)$ also depend on the residue of $n \bmod 8$, as they do for $n$ odd, then one can Fourier analyse the coefficients in order to express them in terms of the complex 8 -th roots of unity to obtain a single formula, as in [3, Prop. 3\&5]. So while the formulae themselves are not periodic in $n$, it may be that the coefficients of each $\rho_{n}\left(\delta_{i}\right)$ featured in each $F_{2}\left(n, t_{1}, t_{2}, t_{3}, t_{4}\right)$ are periodic in $n$.

4.2.4 An alternative proof of general formulae. We now provide another proof of Theorem 8 which relies on the alternative parameterisation of $T_{4}\left(a_{0}^{2}+a_{0}\right)$ and a generalisation of the transform (24). Observe that the functions $f_{0}, \ldots, f_{m-1}: \mathbb{F}_{2^{n}} \rightarrow \mathbb{F}_{2}$ in $\S 3.1$ may be replaced by functions $f_{0}, \ldots, f_{m-1}: A \rightarrow \mathbb{F}_{2}$ where $A$ is any relevant domain - and thus for instance any algebraic set - and exactly the same argument holds, provided that $V(\mathbf{0} \cdot \mathbf{f})$ is redefined to be $|A|$.

The conditions $T_{1}(a)=T_{2}(a)=0$ imply that we should set $A=\left\{\left(a_{0}, a_{1}\right) \in\left(\mathbb{F}_{2^{n}}\right)^{2} \mid a_{0}^{3}+a_{0}=a_{1}^{2}+a_{1}\right\}$. Furthermore let $\mathbf{f}=\left(T_{4}\left(a_{0}^{2}+a_{0}\right), T_{3}\left(a_{0}^{2}+a_{0}\right)\right)=\left(T_{1}\left(a_{1}^{3}+a_{1}+a_{0}^{7}+a_{0}^{5}+a_{0}^{3}+a_{0}\right), T_{1}\left(a_{0}^{5}+a_{0}\right)\right)$. Then by the stated generalisation we have

$$
\left[\begin{array}{l}
F_{2}(n, 0,0,0,0) \\
F_{2}(n, 0,0,1,0) \\
F_{2}(n, 0,0,0,1) \\
F_{2}(n, 0,0,1,1)
\end{array}\right]=\left[\begin{array}{l}
N(\mathbf{0}) \\
N(\mathbf{1}) \\
N(\mathbf{2}) \\
N(\mathbf{3})
\end{array}\right]=\frac{1}{8}\left[\begin{array}{rrrr}
-1 & 1 & 1 & 1 \\
1 & -1 & 1 & -1 \\
1 & 1 & -1 & -1 \\
1 & -1 & -1 & 1
\end{array}\right]\left[\begin{array}{l}
V(\mathbf{0} \cdot \mathbf{f}) \\
V(\mathbf{1} \cdot \mathbf{f}) \\
V(\mathbf{2} \cdot \mathbf{f}) \\
V(\mathbf{3} \cdot \mathbf{f})
\end{array}\right],
$$

where we have a factor of $1 / 8$ rather than $1 / 2$ because of the two factors of $1 / 2$ arising from the introduction of the variables $a_{0}$ and $a_{1}$ defining $A$. Note that $V(\mathbf{0} \cdot \mathbf{f})=|A|=2^{n}-\rho_{n}\left(\delta_{2,1}\right)$. For $1 \leq i \leq 3$ let $\mathbf{i}=\left(i_{1}, i_{0}\right)$. We thus have:

$$
V(\mathbf{i} \cdot \mathbf{f})=\frac{1}{2} \#\left\{\left(a_{0}, a_{1}, a_{2}\right) \mid a_{0}^{3}+a_{0}=a_{1}^{2}+a_{1}, a_{2}^{2}+a_{2}=i_{1}\left(a_{1}^{3}+a_{1}+a_{0}^{7}+a_{0}^{5}+a_{0}^{3}+a_{0}\right)+i_{0}\left(a_{0}^{5}+a_{0}\right)\right\} .
$$

For $i=1,2$ and 3 these are absolutely irreducible curves of genus 5,7 and 7 respectively, and thus their zeta functions are easier to compute than for those curves arising from the direct method, which have 
genus 17. Combining them as per Eq. (42) gives the formulae in $§ 4.2 .3$; it is therefore a slightly more streamlined argument than the one given there.

Another way of viewing this approach is to take the intersections of (40) and set $r_{0}=r_{1}=i_{0}=i_{1}=0$ and observe that the $i_{2}=i_{3}=0$ condition gives $V(\mathbf{0} \cdot \mathbf{f})=|A|$, without the factor $1 / 2$ present for the other three $V(\mathbf{i} \cdot \mathbf{f})$ because there is no $a_{2}$ variable in this case. The approach is therefore more useful in these cases than the direct method, which can not produce formulae for all $F_{2}\left(n, 0,0, t_{3}, t_{4}\right)$ for all $n \geq 4$, as it only works for $n$ odd in general.

4.2.5 Connection with binary Kloosterman sums. The binary Kloosterman sum $\mathcal{K}_{2^{n}}: \mathbb{F}_{2^{n}} \rightarrow \mathbb{Z}$ can be defined by

$$
\mathcal{K}_{2^{n}}(a)=1+\sum_{x \in \mathbb{F}_{2^{n}}^{\times}}(-1)^{T_{1}\left(x^{-1}+a x\right)} .
$$

Kloosterman sums have applications in cryptography and coding theory, see for example [9, 42]. In particular, zeros of $\mathcal{K}_{2^{n}}$ lead to bent functions from $\mathbb{F}_{2^{2 n}} \rightarrow \mathbb{F}_{2}$ [13]. The following elementary lemma connects Kloosterman sums to a family of elliptic curves.

Lemma 11 ([34]). Let $a \in \mathbb{F}_{2^{n}}^{\times}$and define the elliptic curve $E_{2^{n}}(a)$ over $\mathbb{F}_{2^{n}}$ by

$$
E_{2^{n}}(a): y^{2}+x y=x^{3}+a .
$$

Then $\# E_{2^{n}}(a)=2^{n}+\mathcal{K}_{2^{n}}(a)$.

Computing Kloosterman sum zeros is generally regarded as being difficult, currently taking exponential time (in $n)$ to find a single non-trivial $(a \neq 0)$ zero. Besides the deterministic test due to Ahmadi and Granger [4], which computes the cardinality of the Sylow 2-subgroup of any $E_{2^{n}}(a)$ via point-halving, and thus by Lemma 11 the maximum power of 2 dividing $\mathcal{K}_{2^{n}}(a)$, research has focused on characterising Kloosterman sums modulo small integers $[41,37,18,26,38,21,22,20]$. In order to analyse the expected running time of the algorithm of Ahmadi and Granger, it is necessary to know the distribution of Kloosterman sums which are divisible by successive powers of 2 . Table 1 presents this distribution for $n \leq 13$, which was also presented in [4].

Let $T(n, k)$ denote the $(n, k)$-th entry of Table 1, i.e., the number of $a \in \mathbb{F}_{2^{n}}^{\times}$for which $\# E_{2^{n}}(a)$ is divisible by $2^{k}$. By using a result of Katz and Livné [29] it is possible to express $T(n, k)$ in terms of the class numbers of certain imaginary quadratic fields. However, it remains an open problem to give exact formulae for $k>4$, with the formulae for the first four columns being as follows. Since the orders of all of the elliptic curves in Lemma 11 are divisible by 4 , one has $T(n, 1)=T(n, 2)=2^{n}-1$. One can show that $E_{2^{n}}(a)$ has a point of order 8 if and only if $T_{1}(a)=0$ (see e.g. [51]), hence $T_{2^{n}}(3)=2^{n-1}-1$. Finally, Lisoněk and Moisio proved that $T(n, 4)=\left(2^{n}-(-1+i)^{n}-(-1-i)^{n}\right) / 4-1$, connecting it with the number of points on a supersingular elliptic curve [38, Theorem 3.6].

The following theorem connects the distribution of binary Kloosterman sums mod 32 to the distribution of the first four coefficients of the characteristic polynomial.

Theorem 9 ([20]). Let $a \in \mathbb{F}_{2^{n}}$ with $n \geq 4$ and let $e_{1}, \ldots, e_{4}$ be the coefficients of the characteristic polynomial of a, regarded as integers. Then

$$
\mathcal{K}_{2^{n}}(a) \equiv 28 e_{1}+8 e_{2}+16\left(e_{1} e_{2}+e_{1} e_{3}+e_{4}\right) \quad(\bmod 32) .
$$

Combining Theorems 7 and 9 therefore provides explicit formulae for the distribution of binary Kloosterman sums mod 32, for $n$ odd. Furthermore, combining Theorem 9 with Eq. (41) provides an explicit formula for $\#\left\{a \in \mathbb{F}_{2^{n}} \mid \mathcal{K}_{2^{n}}(a) \equiv 0(\bmod 32)\right\}=T(n, 5)+1$; indeed, this connection was our original motivation for considering the first-four prescribed traces problem. 
Table 1. $T(n, k)=\#\left\{a \in \mathbb{F}_{2^{n}}^{\times} \mid \# E_{2^{n}}(a) \equiv 0\left(\bmod 2^{k}\right)\right\}$

\begin{tabular}{c|c|c|c|c|c|c|c|c|c|c|c|c|c}
$n \backslash k$ & 1 & 2 & 3 & 4 & 5 & 6 & 7 & 8 & 9 & 10 & 11 & 12 & 13 \\
\hline 1 & 1 & 1 & & & & & & & & & & & \\
2 & 3 & 3 & & & & & & & & & & & \\
3 & 7 & 7 & 3 & & & & & & & & & & \\
4 & 15 & 15 & 7 & 5 & & & & & & & & & \\
5 & 31 & 31 & 15 & 5 & 5 & & & & & & & & \\
6 & 63 & 63 & 31 & 15 & 12 & 12 & & & & & & & \\
7 & 127 & 127 & 63 & 35 & 14 & 14 & 14 & & & & & & \\
8 & 255 & 255 & 127 & 55 & 21 & 16 & 16 & 16 & & & & & \\
9 & 511 & 511 & 255 & 135 & 63 & 18 & 18 & 18 & 18 & & & & \\
10 & 1023 & 1023 & 511 & 255 & 125 & 65 & 60 & 60 & 60 & 60 & & & \\
11 & 2047 & 2047 & 1023 & 495 & 253 & 132 & 55 & 55 & 55 & 55 & 55 & & \\
12 & 4095 & 4095 & 2047 & 1055 & 495 & 252 & 84 & 72 & 72 & 72 & 72 & 72 & \\
13 & 8191 & 8191 & 4095 & 2015 & 1027 & 481 & 247 & 52 & 52 & 52 & 52 & 52 & 52
\end{tabular}

Corollary 2. For $n \geq 5$ we have

$$
\begin{aligned}
\#\left\{a \in \mathbb{F}_{2^{n}} \mid \mathcal{K}_{2^{n}}(a) \equiv 0 \quad(\bmod 32)\right\} & =F_{2}(n, 0,0,0,0)+F_{2}(n, 0,0,1,0) \\
& =2^{n-3}-\frac{1}{8}\left(\rho_{n}\left(\delta_{2,1}\right)+2 \rho_{n}\left(\delta_{2,2}\right)+\rho_{n}\left(\delta_{8,1}\right)\right) .
\end{aligned}
$$

We also have:

Theorem 10 ([20]). Let $a \in \mathbb{F}_{2^{n}}$ with $n \geq 6$ and let $e_{1}, \ldots, e_{8}$ be the coefficients of the characteristic polynomial of a, regarded as integers. Then

$$
\begin{aligned}
\mathcal{K}_{2^{n}}(a) & \equiv 28 e_{1}+40 e_{2}+16\left(e_{1} e_{2}+e_{1} e_{3}+e_{4}\right) \\
& +32\left(e_{1} e_{4}+e_{1} e_{5}+e_{1} e_{6}+e_{1} e_{7}+e_{2} e_{3}+e_{2} e_{4}+e_{2} e_{6}+e_{3} e_{5}+e_{1} e_{2} e_{3}+e_{1} e_{2} e_{4}+e_{8}\right) \quad(\bmod 64) .
\end{aligned}
$$

Therefore, if one could solve the first-eight prescribed traces problem, then one could also determine a formula for the entries of the sixth column of Table 1.

\subsection{Computing $F_{2}\left(n, t_{1}, t_{2}, t_{3}, t_{4}, t_{5}\right)$}

We begin by applying the indirect method.

4.3.1 Indirect method. Setting $\mathbf{f}=\left(T_{5}, T_{4}, T_{3}, T_{2}, T_{1}\right)$, by the transform (24) we have

$\left[\begin{array}{l}F_{2}(n, 0,0,0,0,0) \\ F_{2}(n, 1,0,0,0,0) \\ F_{2}(n, 0,1,0,0,0) \\ F_{2}(n, 1,1,0,0,0) \\ F_{2}(n, 0,0,1,0,0) \\ F_{2}(n, 1,0,1,0,0) \\ F_{2}(n, 0,1,1,0,0) \\ F_{2}(n, 1,1,1,0,0) \\ F_{2}(n, 0,0,0,1,0) \\ F_{2}(n, 1,0,0,1,0) \\ F_{2}(n, 0,1,0,1,0) \\ F_{2}(n, 1,1,0,1,0) \\ F_{2}(n, 0,0,1,1,0) \\ F_{2}(n, 1,0,1,1,0) \\ F_{2}(n, 0,1,1,1,0) \\ F_{2}(n, 1,1,1,1,0) \\ F_{2}(n, 0,0,0,0,1) \\ F_{2}(n, 1,0,0,0,1) \\ F_{2}(n, 0,1,0,0,1) \\ F_{2}(n, 1,1,0,0,1) \\ F_{2}(n, 0,0,1,0,1) \\ F_{2}(n, 1,0,1,0,1) \\ F_{2}(n, 0,1,1,0,1) \\ F_{2}(n, 1,1,1,0,1) \\ F_{2}(n, 0,0,0,1,1) \\ F_{2}(n, 1,0,0,1,1) \\ F_{2}(n, 0,1,0,1,1) \\ F_{2}(n, 1,1,0,1,1) \\ F_{2}(n, 0,0,1,1,1) \\ F_{2}(n, 1,0,1,1,1) \\ F_{2}(n, 0,1,1,1,1) \\ F_{2}(n, 1,1,1,1,1)\end{array}\right]=\left[\begin{array}{l}N(\mathbf{0}) \\ N(\mathbf{1}) \\ N(\mathbf{2}) \\ N(\mathbf{3}) \\ N(\mathbf{4}) \\ N(\mathbf{5}) \\ N(\mathbf{6}) \\ N(\mathbf{7}) \\ N(\mathbf{8}) \\ N(\mathbf{9}) \\ N(\mathbf{1 0}) \\ N(\mathbf{1 1}) \\ N(\mathbf{1 2}) \\ N(\mathbf{1 3}) \\ N(\mathbf{1 4}) \\ N(\mathbf{1 5}) \\ N(\mathbf{1 6}) \\ N(\mathbf{1 7}) \\ N(\mathbf{1 8}) \\ N(\mathbf{1 9}) \\ N(\mathbf{2 0}) \\ N(\mathbf{2 1}) \\ N(\mathbf{2 2}) \\ N(\mathbf{2 3}) \\ N(\mathbf{2 4}) \\ N(\mathbf{2 5}) \\ N(\mathbf{2 6}) \\ N(\mathbf{2 7}) \\ N(\mathbf{2 8}) \\ N(\mathbf{2 9}) \\ N(\mathbf{3 0}) \\ N(\mathbf{3 1})\end{array}\right]=\frac{1}{16}$

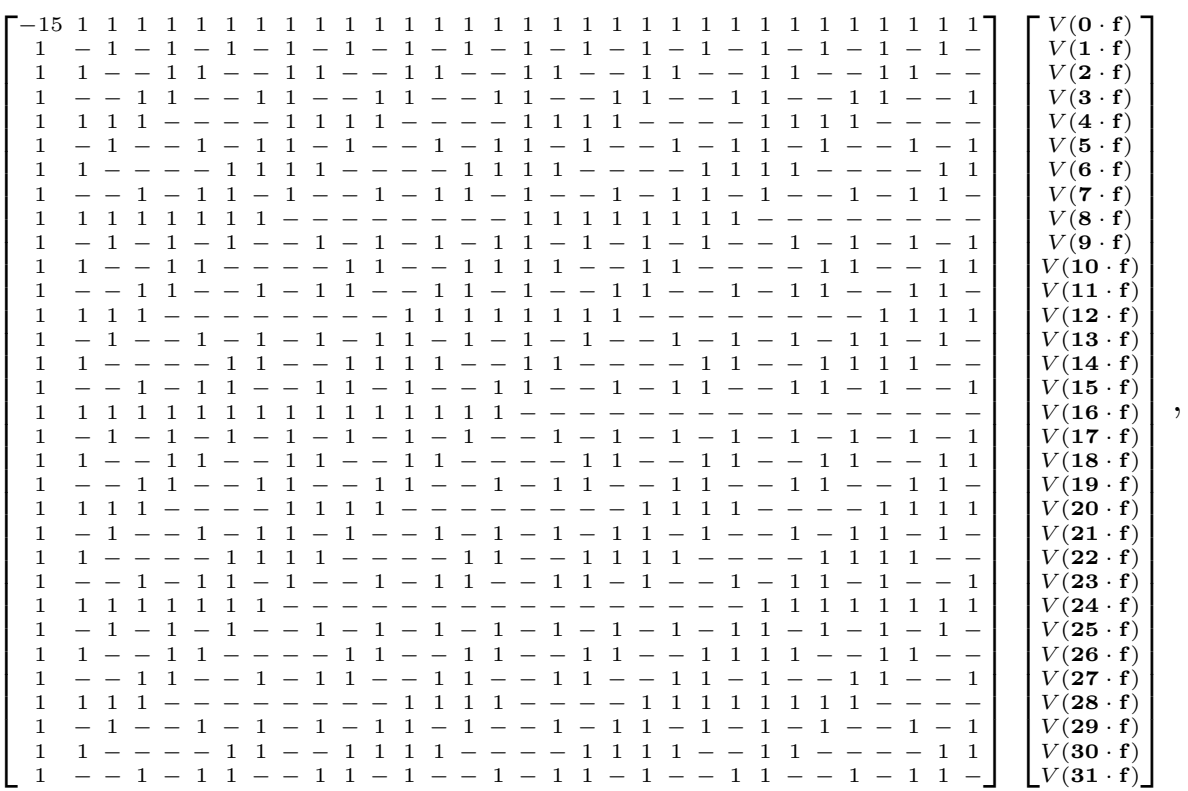


where for reasons of space and in common with Hadamard matrix notation, we represent each -1 simply as a ' - '. To determine $V(\mathbf{i} \cdot \mathbf{f})$ for $16 \leq i \leq 31$, we use Lemma 10 part (5). In particular, setting $\alpha=a_{0}^{2}$ and $\beta=a_{0}$ for $r_{0}=0$, and $\alpha=a_{0}^{2}+a_{0}$ and $\beta=1$ for $r_{0}=1$, and evaluating mod 2 gives the following:

$$
\begin{aligned}
T_{5}\left(a_{0}^{2}+a_{0}+r_{0}\right) & =T_{1}\left(a_{0}^{5}\right) T_{1}\left(a_{0}^{3}\right)+T_{1}\left(a_{0}^{5}\right) T_{1}\left(a_{0}\right)+T_{1}\left(a_{0}^{3}\right) T_{1}\left(a_{0}\right)+r_{0}\left(T_{2}\left(a_{0}^{3}\right)+T_{2}\left(a_{0}\right)+T_{1}\left(a_{0}^{3}\right) T_{1}\left(a_{0}\right)\right) \\
& +T_{1}\left(a_{0}^{9}+a_{0}^{3}+a_{0}+r_{0}\left(a_{0}^{7}+\left(a_{0}^{5}+a_{0}\right)\left(\begin{array}{c}
n \\
2
\end{array}\right)+\left(a_{0}^{3}+a_{0}\right)\left(\begin{array}{l}
n \\
3
\end{array}\right)+\left(\begin{array}{c}
n \\
5
\end{array}\right)\right)\right) .
\end{aligned}
$$

This can be linearised using the same the substitutions that were used for the four coefficient case, namely, $a_{0}=a_{1}^{2}+a_{1}+r_{1}$ and $a_{0}^{3}=a_{2}^{2}+a_{2}+r_{2}$, where $r_{1}, r_{2} \in \mathbb{F}_{2}$ are the traces of $a_{0}$ and $a_{0}^{3}$ respectively. This results in

$$
\begin{aligned}
T_{5}\left(a_{0}^{2}+a_{0}+r_{0}\right) & =T_{1}\left(r_{0}\left(a_{2}^{3}+a_{2}+a_{1}^{3}+a_{1}\right)+a_{0}^{9}+r_{0} a_{0}^{7}+\left(r_{1}+r_{2}\right) a_{0}^{5}+r_{1}+r_{2}+r_{1} r_{2}+r_{0} r_{1} r_{2}\right. \\
& \left.+\left(r_{0} a_{0}^{5}+r_{0} r_{2}\right)\left(\begin{array}{l}
n \\
2
\end{array}\right)+\left(r_{0} r_{1}+r_{0} r_{2}\right)\left(\begin{array}{l}
n \\
3
\end{array}\right)+r_{0}\left(\begin{array}{c}
n \\
5
\end{array}\right)\right) .
\end{aligned}
$$

For $16 \leq i \leq 31$ let $\mathbf{i}=\left(i_{4}, i_{3}, i_{2}, i_{1}, i_{0}\right)$. The curves we are interested in are given by the following intersections:

$$
\begin{aligned}
a_{3}^{2}+a_{3} & =i_{4}\left(r_{0}\left(a_{2}^{3}+a_{2}+a_{1}^{3}+a_{1}\right)+a_{0}^{9}+r_{0} a_{0}^{7}+\left(r_{1}+r_{2}\right) a_{0}^{5}+r_{1}+r_{2}+r_{1} r_{2}+r_{0} r_{1} r_{2}\right. \\
& \left.+\left(r_{0} a_{0}^{5}+r_{0} r_{2}\right)\left(\begin{array}{c}
n \\
2
\end{array}\right)+\left(r_{0} r_{1}+r_{0} r_{2}\right)\left(\begin{array}{c}
n \\
3
\end{array}\right)+r_{0}\left(\begin{array}{c}
n \\
5
\end{array}\right)\right) \\
& +i_{3}\left(a_{2}^{3}+a_{2}+a_{1}^{3}+a_{1}+a_{0}^{7}+a_{0}^{5}+\left(r_{0}+1\right)\left(r_{1}+r_{2}\right)\left(\begin{array}{c}
n \\
2
\end{array}\right)+r_{0} r_{1}+r_{0} r_{2}+r_{1} r_{2}+r_{2}+r_{0}\left(\begin{array}{c}
n \\
4
\end{array}\right)\right) \\
& +i_{2}\left(a_{0}^{5}+a_{0}+r_{0}\left(a_{0}^{3}+a_{0}+\left(\begin{array}{c}
n \\
3
\end{array}\right)\right)\right)+i_{1}\left(a_{0}^{3}+a_{0}+r_{0}\left(\begin{array}{c}
n \\
2
\end{array}\right)\right)+i_{0} r_{0} \\
a_{0} & =a_{1}^{2}+a_{1}+r_{1} \\
a_{0}^{3} & =a_{2}^{2}+a_{2}+r_{2} .
\end{aligned}
$$

For each $16 \leq i \leq 31$ the genus of all of these absolutely irreducible curves is 18 . Corollary 1 implies that mod 2 one has

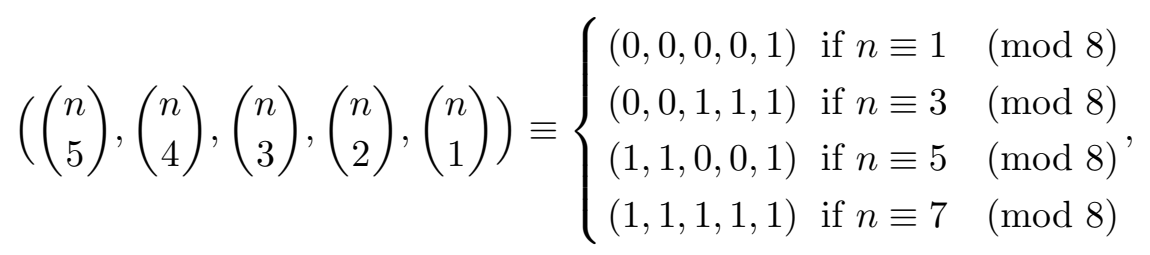

and hence there are four cases to consider when computing the zeta functions of each of the curves (44). In order to express $F_{2}\left(n, t_{1}, t_{2}, t_{3}, t_{4}, t_{5}\right)$ compactly, we define the following polynomial:

$$
\delta_{8,3}=X^{8}+2 X^{7}+2 X^{6}-4 X^{4}+8 X^{2}+16 X+16,
$$

which is the characteristic polynomial of Frobenius of the supersingular curve

$$
C_{8,3} / \mathbb{F}_{2}: y^{2}+y=x^{9}+x^{5} .
$$

As with the four coefficient case there are several other even polynomials which occur as factors of the characteristic polynomial of Frobenius of the above curves, which can hence be ignored for $n$ odd.

We used Magma V22.2-3 to compute the zeta functions of the curves (44), which took just under 15 minutes on a $2.0 \mathrm{GHz}$ AMD Opteron computer and leads to the following theorem ${ }^{\dagger}$.

\footnotetext{
${ }^{\dagger}$ See F2 (n, t1 $\left., t 2, t 3, t 4, t 5\right) . m$.
} 
Theorem 11. For $n \geq 5$ we have

$F_{2}(n, 0,0,0,0,0)=2^{n-5}-\frac{1}{32}\left(5 \rho_{n}\left(\delta_{2,1}\right)+5 \rho_{n}\left(\delta_{4,1}\right)+2 \rho_{n}\left(\delta_{8,1}\right)+\rho_{n}\left(\delta_{8,3}\right)\right) \quad$ if $n \equiv 1,3,5,7 \quad(\bmod 8)$ $F_{2}(n, 1,0,0,0,0)=2^{n-5}-\frac{1}{32} \cdot\left\{\begin{array}{lll}5 \rho_{n}\left(\delta_{2,1}\right)+5 \rho_{n}\left(\delta_{4,1}\right)+2 \rho_{n}\left(\delta_{8,1}\right)+\rho_{n}\left(\delta_{8,3}\right) & \text { if } n \equiv 1 \quad(\bmod 8) \\ 3 \rho_{n}\left(\delta_{2,1}\right)-3 \rho_{n}\left(\delta_{4,1}\right)+2 \rho_{n}\left(\delta_{8,2}\right)-\rho_{n}\left(\delta_{8,3}\right) & \text { if } n \equiv 3 & (\bmod 8) \\ \rho_{n}\left(\delta_{2,1}\right)+\rho_{n}\left(\delta_{4,1}\right)-2 \rho_{n}\left(\delta_{8,1}\right)+\rho_{n}\left(\delta_{8,3}\right) & \text { if } n \equiv 5 & (\bmod 8) \\ -\rho_{n}\left(\delta_{2,1}\right)+\rho_{n}\left(\delta_{4,1}\right)-2 \rho_{n}\left(\delta_{8,2}\right)-\rho_{n}\left(\delta_{8,3}\right) & \text { if } n \equiv 7 & (\bmod 8)\end{array}\right.$

$F_{2}(n, 0,1,0,0,0)=2^{n-5}-\frac{1}{32} \cdot \begin{cases}-\rho_{n}\left(\delta_{2,1}\right)+\rho_{n}\left(\delta_{4,1}\right)-2 \rho_{n}\left(\delta_{8,2}\right)-\rho_{n}\left(\delta_{8,3}\right) & \text { if } n \equiv 1,5 \quad(\bmod 8) \\ 3 \rho_{n}\left(\delta_{2,1}\right)-3 \rho_{n}\left(\delta_{4,1}\right)+2 \rho_{n}\left(\delta_{8,2}\right)-\rho_{n}\left(\delta_{8,3}\right) & \text { if } n \equiv 3,7 \quad(\bmod 8)\end{cases}$

$F_{2}(n, 1,1,0,0,0)=2^{n-5}-\frac{1}{32} \cdot\left\{\begin{array}{lll}-3 \rho_{n}\left(\delta_{2,1}\right)-3 \rho_{n}\left(\delta_{4,1}\right)+2 \rho_{n}\left(\delta_{8,1}\right)+\rho_{n}\left(\delta_{8,3}\right) & \text { if } n \equiv 1 \quad(\bmod 8) \\ -\rho_{n}\left(\delta_{2,1}\right)+\rho_{n}\left(\delta_{4,1}\right)-2 \rho_{n}\left(\delta_{8,2}\right)-\rho_{n}\left(\delta_{8,3}\right) & \text { if } n \equiv 3 \quad(\bmod 8) \\ \rho_{n}\left(\delta_{2,1}\right)+\rho_{n}\left(\delta_{4,1}\right)-2 \rho_{n}\left(\delta_{8,1}\right)+\rho_{n}\left(\delta_{8,3}\right) & \text { if } n \equiv 5 \quad(\bmod 8) \\ 3 \rho_{n}\left(\delta_{2,1}\right)-3 \rho_{n}\left(\delta_{4,1}\right)+2 \rho_{n}\left(\delta_{8,2}\right)-\rho_{n}\left(\delta_{8,3}\right) & \text { if } n \equiv 7 \quad(\bmod 8)\end{array}\right.$

$F_{2}(n, 0,0,1,0,0)=2^{n-5}-\frac{1}{32}\left(-\rho_{n}\left(\delta_{2,1}\right)+\rho_{n}\left(\delta_{4,1}\right)-2 \rho_{n}\left(\delta_{8,2}\right)-\rho_{n}\left(\delta_{8,3}\right)\right)$ if $n \equiv 1,3,5,7 \quad(\bmod 8)$

$F_{2}(n, 1,0,1,0,0)=2^{n-5}-\frac{1}{32} \cdot\left\{\begin{array}{lll}-3 \rho_{n}\left(\delta_{2,1}\right)-3 \rho_{n}\left(\delta_{4,1}\right)+2 \rho_{n}\left(\delta_{8,1}\right)+\rho_{n}\left(\delta_{8,3}\right) & \text { if } n \equiv 1 \quad(\bmod 8) \\ -5 \rho_{n}\left(\delta_{2,1}\right)+5 \rho_{n}\left(\delta_{4,1}\right)+2 \rho_{n}\left(\delta_{8,2}\right)-\rho_{n}\left(\delta_{8,3}\right) & \text { if } n \equiv 3 \quad(\bmod 8) \\ \rho_{n}\left(\delta_{2,1}\right)+\rho_{n}\left(\delta_{4,1}\right)-2 \rho_{n}\left(\delta_{8,1}\right)+\rho_{n}\left(\delta_{8,3}\right) & \text { if } n \equiv 5 \quad(\bmod 8) \\ -\rho_{n}\left(\delta_{2,1}\right)+\rho_{n}\left(\delta_{4,1}\right)-2 \rho_{n}\left(\delta_{8,2}\right)-\rho_{n}\left(\delta_{8,3}\right) & \text { if } n \equiv 7 \quad(\bmod 8)\end{array}\right.$

$F_{2}(n, 0,1,1,0,0)=2^{n-5}-\frac{1}{32} \cdot\left\{\begin{array}{lll}-\rho_{n}\left(\delta_{2,1}\right)+\rho_{n}\left(\delta_{4,1}\right)-2 \rho_{n}\left(\delta_{8,2}\right)-\rho_{n}\left(\delta_{8,3}\right) & \text { if } n \equiv 1,5 \quad(\bmod 8) \\ -5 \rho_{n}\left(\delta_{2,1}\right)+5 \rho_{n}\left(\delta_{4,1}\right)+2 \rho_{n}\left(\delta_{8,2}\right)-\rho_{n}\left(\delta_{8,3}\right) & \text { if } n \equiv 3,7 & (\bmod 8)\end{array}\right.$

$F_{2}(n, 1,1,1,0,0)=2^{n-5}-\frac{1}{32} \cdot\left\{\begin{array}{lll}3 \rho_{n}\left(\delta_{2,1}\right)-3 \rho_{n}\left(\delta_{4,1}\right)+2 \rho_{n}\left(\delta_{8,2}\right)-\rho_{n}\left(\delta_{8,3}\right) & \text { if } n \equiv 1 \quad(\bmod 8) \\ 5 \rho_{n}\left(\delta_{2,1}\right)+5 \rho_{n}\left(\delta_{4,1}\right)+2 \rho_{n}\left(\delta_{8,1}\right)+\rho_{n}\left(\delta_{8,3}\right) & \text { if } n \equiv 3 \quad(\bmod 8) \\ -\rho_{n}\left(\delta_{2,1}\right)+\rho_{n}\left(\delta_{4,1}\right)-2 \rho_{n}\left(\delta_{8,2}\right)-\rho_{n}\left(\delta_{8,3}\right) & \text { if } n \equiv 5 \quad(\bmod 8) \\ \rho_{n}\left(\delta_{2,1}\right)+\rho_{n}\left(\delta_{4,1}\right)-2 \rho_{n}\left(\delta_{8,1}\right)+\rho_{n}\left(\delta_{8,3}\right) & \text { if } n \equiv 7 & (\bmod 8)\end{array}\right.$

$F_{2}(n, 0,0,0,1,0)=2^{n-5}-\frac{1}{32}\left(\rho_{n}\left(\delta_{2,1}\right)+\rho_{n}\left(\delta_{4,1}\right)-2 \rho_{n}\left(\delta_{8,1}\right)+\rho_{n}\left(\delta_{8,3}\right)\right)$ if $n \equiv 1,3,5,7 \quad(\bmod 8)$

$F_{2}(n, 1,0,0,1,0)=2^{n-5}-\frac{1}{32} \cdot\left\{\begin{array}{lll}-\rho_{n}\left(\delta_{2,1}\right)+\rho_{n}\left(\delta_{4,1}\right)-2 \rho_{n}\left(\delta_{8,2}\right)-\rho_{n}\left(\delta_{8,3}\right) & \text { if } n \equiv 1 & (\bmod 8) \\ -3 \rho_{n}\left(\delta_{2,1}\right)-3 \rho_{n}\left(\delta_{4,1}\right)+2 \rho_{n}\left(\delta_{8,1}\right)+\rho_{n}\left(\delta_{8,3}\right) & \text { if } n \equiv 3 \quad(\bmod 8) \\ 3 \rho_{n}\left(\delta_{2,1}\right)-3 \rho_{n}\left(\delta_{4,1}\right)+2 \rho_{n}\left(\delta_{8,2}\right)-\rho_{n}\left(\delta_{8,3}\right) & \text { if } n \equiv 5 & (\bmod 8) \\ \rho_{n}\left(\delta_{2,1}\right)+\rho_{n}\left(\delta_{4,1}\right)-2 \rho_{n}\left(\delta_{8,1}\right)+\rho_{n}\left(\delta_{8,3}\right) & \text { if } n \equiv 7 & (\bmod 8)\end{array}\right.$

$F_{2}(n, 0,1,0,1,0)=2^{n-5}-\frac{1}{32} \cdot \begin{cases}3 \rho_{n}\left(\delta_{2,1}\right)-3 \rho_{n}\left(\delta_{4,1}\right)+2 \rho_{n}\left(\delta_{8,2}\right)-\rho_{n}\left(\delta_{8,3}\right) & \text { if } n \equiv 1,5 \quad(\bmod 8) \\ -\rho_{n}\left(\delta_{2,1}\right)+\rho_{n}\left(\delta_{4,1}\right)-2 \rho_{n}\left(\delta_{8,2}\right)-\rho_{n}\left(\delta_{8,3}\right) & \text { if } n \equiv 3,7 \quad(\bmod 8)\end{cases}$

$F_{2}(n, 1,1,0,1,0)=2^{n-5}-\frac{1}{32} \cdot\left\{\begin{array}{lll}-\rho_{n}\left(\delta_{2,1}\right)+\rho_{n}\left(\delta_{4,1}\right)-2 \rho_{n}\left(\delta_{8,2}\right)-\rho_{n}\left(\delta_{8,3}\right) & \text { if } n \equiv 1 \quad(\bmod 8) \\ \rho_{n}\left(\delta_{2,1}\right)+\rho_{n}\left(\delta_{4,1}\right)-2 \rho_{n}\left(\delta_{8,1}\right)+\rho_{n}\left(\delta_{8,3}\right) & \text { if } n \equiv 3 \quad(\bmod 8) \\ -5 \rho_{n}\left(\delta_{2,1}\right)+5 \rho_{n}\left(\delta_{4,1}\right)+2 \rho_{n}\left(\delta_{8,2}\right)-\rho_{n}\left(\delta_{8,3}\right) & \text { if } n \equiv 5 \quad(\bmod 8) \\ -3 \rho_{n}\left(\delta_{2,1}\right)-3 \rho_{n}\left(\delta_{4,1}\right)+2 \rho_{n}\left(\delta_{8,1}\right)+\rho_{n}\left(\delta_{8,3}\right) & \text { if } n \equiv 7 \quad(\bmod 8)\end{array}\right.$

$F_{2}(n, 0,0,1,1,0)=2^{n-5}-\frac{1}{32}\left(3 \rho_{n}\left(\delta_{2,1}\right)-3 \rho_{n}\left(\delta_{4,1}\right)+2 \rho_{n}\left(\delta_{8,2}\right)-\rho_{n}\left(\delta_{8,3}\right)\right)$ if $n \equiv 1,3,5,7 \quad(\bmod 8)$

$F_{2}(n, 1,0,1,1,0)=2^{n-5}-\frac{1}{32} \cdot\left\{\begin{array}{lll}3 \rho_{n}\left(\delta_{2,1}\right)-3 \rho_{n}\left(\delta_{4,1}\right)+2 \rho_{n}\left(\delta_{8,2}\right)-\rho_{n}\left(\delta_{8,3}\right) & \text { if } n \equiv 1 \quad(\bmod 8) \\ \rho_{n}\left(\delta_{2,1}\right)+\rho_{n}\left(\delta_{4,1}\right)-2 \rho_{n}\left(\delta_{8,1}\right)+\rho_{n}\left(\delta_{8,3}\right) & \text { if } n \equiv 3 \quad(\bmod 8) \\ -\rho_{n}\left(\delta_{2,1}\right)+\rho_{n}\left(\delta_{4,1}\right)-2 \rho_{n}\left(\delta_{8,2}\right)-\rho_{n}\left(\delta_{8,3}\right) & \text { if } n \equiv 5 \quad(\bmod 8) \\ -3 \rho_{n}\left(\delta_{2,1}\right)-3 \rho_{n}\left(\delta_{4,1}\right)+2 \rho_{n}\left(\delta_{8,1}\right)+\rho_{n}\left(\delta_{8,3}\right) & \text { if } n \equiv 7 \quad(\bmod 8)\end{array}\right.$

$F_{2}(n, 0,1,1,1,0)=2^{n-5}-\frac{1}{32} \cdot\left\{\begin{array}{lll}-5 \rho_{n}\left(\delta_{2,1}\right)+5 \rho_{n}\left(\delta_{4,1}\right)+2 \rho_{n}\left(\delta_{8,2}\right)-\rho_{n}\left(\delta_{8,3}\right) & \text { if } n \equiv 1,5 \quad(\bmod 8) \\ -\rho_{n}\left(\delta_{2,1}\right)+\rho_{n}\left(\delta_{4,1}\right)-2 \rho_{n}\left(\delta_{8,2}\right)-\rho_{n}\left(\delta_{8,3}\right) & \text { if } n \equiv 3,7 \quad(\bmod 8)\end{array}\right.$

$F_{2}(n, 1,1,1,1,0)=2^{n-5}-\frac{1}{32} \cdot\left\{\begin{array}{lll}-3 \rho_{n}\left(\delta_{2,1}\right)-3 \rho_{n}\left(\delta_{4,1}\right)+2 \rho_{n}\left(\delta_{8,1}\right)+\rho_{n}\left(\delta_{8,3}\right) & \text { if } n \equiv 1 \quad(\bmod 8) \\ -\rho_{n}\left(\delta_{2,1}\right)+\rho_{n}\left(\delta_{4,1}\right)-2 \rho_{n}\left(\delta_{8,2}\right)-\rho_{n}\left(\delta_{8,3}\right) & \text { if } n \equiv 3 \quad(\bmod 8) \\ \rho_{n}\left(\delta_{2,1}\right)+\rho_{n}\left(\delta_{4,1}\right)-2 \rho_{n}\left(\delta_{8,1}\right)+\rho_{n}\left(\delta_{8,3}\right) & \text { if } n \equiv 5 \quad(\bmod 8) \\ 3 \rho_{n}\left(\delta_{2,1}\right)-3 \rho_{n}\left(\delta_{4,1}\right)+2 \rho_{n}\left(\delta_{8,2}\right)-\rho_{n}\left(\delta_{8,3}\right) & \text { if } n \equiv 7 \quad(\bmod 8)\end{array}\right.$ 
$F_{2}(n, 0,0,0,0,1)=2^{n-5}-\frac{1}{32}\left(3 \rho_{n}\left(\delta_{2,1}\right)-3 \rho_{n}\left(\delta_{4,1}\right)+2 \rho_{n}\left(\delta_{8,2}\right)-\rho_{n}\left(\delta_{8,3}\right)\right) \quad$ if $n \equiv 1,3,5,7 \quad(\bmod 8)$

$F_{2}(n, 1,0,0,0,1)=2^{n-5}-\frac{1}{32} \cdot\left\{\begin{array}{lll}3 \rho_{n}\left(\delta_{2,1}\right)-3 \rho_{n}\left(\delta_{4,1}\right)+2 \rho_{n}\left(\delta_{8,2}\right)-\rho_{n}\left(\delta_{8,3}\right) & \text { if } n \equiv 1 \quad(\bmod 8) \\ \rho_{n}\left(\delta_{2,1}\right)+\rho_{n}\left(\delta_{4,1}\right)-2 \rho_{n}\left(\delta_{8,1}\right)+\rho_{n}\left(\delta_{8,3}\right) & \text { if } n \equiv 3 \quad(\bmod 8) \\ -\rho_{n}\left(\delta_{2,1}\right)+\rho_{n}\left(\delta_{4,1}\right)-2 \rho_{n}\left(\delta_{8,2}\right)-\rho_{n}\left(\delta_{8,3}\right) & \text { if } n \equiv 5 \quad(\bmod 8) \\ -3 \rho_{n}\left(\delta_{2,1}\right)-3 \rho_{n}\left(\delta_{4,1}\right)+2 \rho_{n}\left(\delta_{8,1}\right)+\rho_{n}\left(\delta_{8,3}\right) & \text { if } n \equiv 7 \quad(\bmod 8)\end{array}\right.$

$F_{2}(n, 0,1,0,0,1)=2^{n-5}-\frac{1}{32} \cdot\left\{\begin{array}{lll}-3 \rho_{n}\left(\delta_{2,1}\right)-3 \rho_{n}\left(\delta_{4,1}\right)+2 \rho_{n}\left(\delta_{8,1}\right)+\rho_{n}\left(\delta_{8,3}\right) & \text { if } n \equiv 1,5 \quad(\bmod 8) \\ \rho_{n}\left(\delta_{2,1}\right)+\rho_{n}\left(\delta_{4,1}\right)-2 \rho_{n}\left(\delta_{8,1}\right)+\rho_{n}\left(\delta_{8,3}\right) & \text { if } n \equiv 3,7 \quad(\bmod 8)\end{array}\right.$

$F_{2}(n, 1,1,0,0,1)=2^{n-5}-\frac{1}{32} \cdot\left\{\begin{array}{lll}-5 \rho_{n}\left(\delta_{2,1}\right)+5 \rho_{n}\left(\delta_{4,1}\right)+2 \rho_{n}\left(\delta_{8,2}\right)-\rho_{n}\left(\delta_{8,3}\right) & \text { if } n \equiv 1 & (\bmod 8) \\ -3 \rho_{n}\left(\delta_{2,1}\right)-3 \rho_{n}\left(\delta_{4,1}\right)+2 \rho_{n}\left(\delta_{8,1}\right)+\rho_{n}\left(\delta_{8,3}\right) & \text { if } n \equiv 3 & (\bmod 8) \\ -\rho_{n}\left(\delta_{2,1}\right)+\rho_{n}\left(\delta_{4,1}\right)-2 \rho_{n}\left(\delta_{8,2}\right)-\rho_{n}\left(\delta_{8,3}\right) & \text { if } n \equiv 5 & (\bmod 8) \\ \rho_{n}\left(\delta_{2,1}\right)+\rho_{n}\left(\delta_{4,1}\right)-2 \rho_{n}\left(\delta_{8,1}\right)+\rho_{n}\left(\delta_{8,3}\right) & \text { if } n \equiv 7 & (\bmod 8)\end{array}\right.$

$F_{2}(n, 0,0,1,0,1)=2^{n-5}-\frac{1}{32}\left(-3 \rho_{n}\left(\delta_{2,1}\right)-3 \rho_{n}\left(\delta_{4,1}\right)+2 \rho_{n}\left(\delta_{8,1}\right)+\rho_{n}\left(\delta_{8,3}\right)\right) \quad$ if $n \equiv 1,3,5,7 \quad(\bmod 8)$

$F_{2}(n, 1,0,1,0,1)=2^{n-5}-\frac{1}{32} \cdot\left\{\begin{array}{lll}-\rho_{n}\left(\delta_{2,1}\right)+\rho_{n}\left(\delta_{4,1}\right)-2 \rho_{n}\left(\delta_{8,2}\right)-\rho_{n}\left(\delta_{8,3}\right) & \text { if } n \equiv 1 \quad(\bmod 8) \\ -3 \rho_{n}\left(\delta_{2,1}\right)-3 \rho_{n}\left(\delta_{4,1}\right)+2 \rho_{n}\left(\delta_{8,1}\right)+\rho_{n}\left(\delta_{8,3}\right) & \text { if } n \equiv 3 \quad(\bmod 8) \\ 3 \rho_{n}\left(\delta_{2,1}\right)-3 \rho_{n}\left(\delta_{4,1}\right)+2 \rho_{n}\left(\delta_{8,2}\right)-\rho_{n}\left(\delta_{8,3}\right) & \text { if } n \equiv 5 \quad(\bmod 8) \\ \rho_{n}\left(\delta_{2,1}\right)+\rho_{n}\left(\delta_{4,1}\right)-2 \rho_{n}\left(\delta_{8,1}\right)+\rho_{n}\left(\delta_{8,3}\right) & \text { if } n \equiv 7 \quad(\bmod 8)\end{array}\right.$

$F_{2}(n, 0,1,1,0,1)=2^{n-5}-\frac{1}{32} \cdot \begin{cases}\rho_{n}\left(\delta_{2,1}\right)+\rho_{n}\left(\delta_{4,1}\right)-2 \rho_{n}\left(\delta_{8,1}\right)+\rho_{n}\left(\delta_{8,3}\right) & \text { if } n \equiv 1,5 \quad(\bmod 8) \\ -3 \rho_{n}\left(\delta_{2,1}\right)-3 \rho_{n}\left(\delta_{4,1}\right)+2 \rho_{n}\left(\delta_{8,1}\right)+\rho_{n}\left(\delta_{8,3}\right) & \text { if } n \equiv 3,7\end{cases}$

$F_{2}(n, 1,1,1,0,1)=2^{n-5}-\frac{1}{32} \cdot\left\{\begin{array}{lll}\rho_{n}\left(\delta_{2,1}\right)+\rho_{n}\left(\delta_{4,1}\right)-2 \rho_{n}\left(\delta_{8,1}\right)+\rho_{n}\left(\delta_{8,3}\right) & \text { if } n \equiv 1 \quad(\bmod 8) \\ 3 \rho_{n}\left(\delta_{2,1}\right)-3 \rho_{n}\left(\delta_{4,1}\right)+2 \rho_{n}\left(\delta_{8,2}\right)-\rho_{n}\left(\delta_{8,3}\right) & \text { if } n \equiv 3 \quad(\bmod 8) \\ -3 \rho_{n}\left(\delta_{2,1}\right)-3 \rho_{n}\left(\delta_{4,1}\right)+2 \rho_{n}\left(\delta_{8,1}\right)+\rho_{n}\left(\delta_{8,3}\right) & \text { if } n \equiv 5 \quad(\bmod 8) \\ -\rho_{n}\left(\delta_{2,1}\right)+\rho_{n}\left(\delta_{4,1}\right)-2 \rho_{n}\left(\delta_{8,2}\right)-\rho_{n}\left(\delta_{8,3}\right) & \text { if } n \equiv 7 \quad(\bmod 8)\end{array}\right.$

$F_{2}(n, 0,0,0,1,1)=2^{n-5}-\frac{1}{32}\left(-\rho_{n}\left(\delta_{2,1}\right)+\rho_{n}\left(\delta_{4,1}\right)-2 \rho_{n}\left(\delta_{8,2}\right)-\rho_{n}\left(\delta_{8,3}\right)\right) \quad$ if $n \equiv 1,3,5,7 \quad(\bmod 8)$

$F_{2}(n, 1,0,0,1,1)=2^{n-5}-\frac{1}{32} \cdot\left\{\begin{array}{lll}\rho_{n}\left(\delta_{2,1}\right)+\rho_{n}\left(\delta_{4,1}\right)-2 \rho_{n}\left(\delta_{8,1}\right)+\rho_{n}\left(\delta_{8,3}\right) & \text { if } n \equiv 1 \quad(\bmod 8) \\ -\rho_{n}\left(\delta_{2,1}\right)+\rho_{n}\left(\delta_{4,1}\right)-2 \rho_{n}\left(\delta_{8,2}\right)-\rho_{n}\left(\delta_{8,3}\right) & \text { if } n \equiv 3 \quad(\bmod 8) \\ 5 \rho_{n}\left(\delta_{2,1}\right)+5 \rho_{n}\left(\delta_{4,1}\right)+2 \rho_{n}\left(\delta_{8,1}\right)+\rho_{n}\left(\delta_{8,3}\right) & \text { if } n \equiv 5 \quad(\bmod 8) \\ 3 \rho_{n}\left(\delta_{2,1}\right)-3 \rho_{n}\left(\delta_{4,1}\right)+2 \rho_{n}\left(\delta_{8,2}\right)-\rho_{n}\left(\delta_{8,3}\right) & \text { if } n \equiv 7 \quad(\bmod 8)\end{array}\right.$

$F_{2}(n, 0,1,0,1,1)=2^{n-5}-\frac{1}{32} \cdot \begin{cases}\rho_{n}\left(\delta_{2,1}\right)+\rho_{n}\left(\delta_{4,1}\right)-2 \rho_{n}\left(\delta_{8,1}\right)+\rho_{n}\left(\delta_{8,3}\right) & \text { if } n \equiv 1,5 \quad(\bmod 8) \\ -3 \rho_{n}\left(\delta_{2,1}\right)-3 \rho_{n}\left(\delta_{4,1}\right)+2 \rho_{n}\left(\delta_{8,1}\right)+\rho_{n}\left(\delta_{8,3}\right) & \text { if } n \equiv 3,7 \quad(\bmod 8)\end{cases}$

$F_{2}(n, 1,1,0,1,1)=2^{n-5}-\frac{1}{32} \cdot\left\{\begin{array}{lll}\rho_{n}\left(\delta_{2,1}\right)+\rho_{n}\left(\delta_{4,1}\right)-2 \rho_{n}\left(\delta_{8,1}\right)+\rho_{n}\left(\delta_{8,3}\right) & \text { if } n \equiv 1 \quad(\bmod 8) \\ 3 \rho_{n}\left(\delta_{2,1}\right)-3 \rho_{n}\left(\delta_{4,1}\right)+2 \rho_{n}\left(\delta_{8,2}\right)-\rho_{n}\left(\delta_{8,3}\right) & \text { if } n \equiv 3 \quad(\bmod 8) \\ -3 \rho_{n}\left(\delta_{2,1}\right)-3 \rho_{n}\left(\delta_{4,1}\right)+2 \rho_{n}\left(\delta_{8,1}\right)+\rho_{n}\left(\delta_{8,3}\right) & \text { if } n \equiv 5 \quad(\bmod 8) \\ -\rho_{n}\left(\delta_{2,1}\right)+\rho_{n}\left(\delta_{4,1}\right)-2 \rho_{n}\left(\delta_{8,2}\right)-\rho_{n}\left(\delta_{8,3}\right) & \text { if } n \equiv 7 \quad(\bmod 8)\end{array}\right.$

$F_{2}(n, 0,0,1,1,1)=2^{n-5}-\frac{1}{32}\left(\rho_{n}\left(\delta_{2,1}\right)+\rho_{n}\left(\delta_{4,1}\right)-2 \rho_{n}\left(\delta_{8,1}\right)+\rho_{n}\left(\delta_{8,3}\right)\right) \quad$ if $n \equiv 1,3,5,7 \quad(\bmod 8)$

$F(n, 1,0,1,1,1)=2^{n-5}-\frac{1}{32} \cdot\left\{\begin{array}{lll}\rho_{n}\left(\delta_{2,1}\right)+\rho_{n}\left(\delta_{4,1}\right)-2 \rho_{n}\left(\delta_{8,1}\right)+\rho_{n}\left(\delta_{8,3}\right) & \text { if } n \equiv 1 \quad(\bmod 8) \\ -\rho_{n}\left(\delta_{2,1}\right)+\rho_{n}\left(\delta_{4,1}\right)-2 \rho_{n}\left(\delta_{8,2}\right)-\rho_{n}\left(\delta_{8,3}\right) & \text { if } n \equiv 3 \quad(\bmod 8) \\ -3 \rho_{n}\left(\delta_{2,1}\right)-3 \rho_{n}\left(\delta_{4,1}\right)+2 \rho_{n}\left(\delta_{8,1}\right)+\rho_{n}\left(\delta_{8,3}\right) & \text { if } n \equiv 5 \quad(\bmod 8) \\ -5 \rho_{n}\left(\delta_{2,1}\right)+5 \rho_{n}\left(\delta_{4,1}\right)+2 \rho_{n}\left(\delta_{8,2}\right)-\rho_{n}\left(\delta_{8,3}\right) & \text { if } n \equiv 7 \quad(\bmod 8)\end{array}\right.$

$F_{2}(n, 0,1,1,1,1)=2^{n-5}-\frac{1}{32} \cdot\left\{\begin{array}{lll}-3 \rho_{n}\left(\delta_{2,1}\right)-3 \rho_{n}\left(\delta_{4,1}\right)+2 \rho_{n}\left(\delta_{8,1}\right)+\rho_{n}\left(\delta_{8,3}\right) & \text { if } n \equiv 1,5 \quad(\bmod 8) \\ \rho_{n}\left(\delta_{2,1}\right)+\rho_{n}\left(\delta_{4,1}\right)-2 \rho_{n}\left(\delta_{8,1}\right)+\rho_{n}\left(\delta_{8,3}\right) & \text { if } n \equiv 3,7 & (\bmod 8)\end{array}\right.$

$F_{2}(n, 1,1,1,1,1)=2^{n-5}-\frac{1}{32} \cdot\left\{\begin{array}{lll}-\rho_{n}\left(\delta_{2,1}\right)+\rho_{n}\left(\delta_{4,1}\right)-2 \rho_{n}\left(\delta_{8,2}\right)-\rho_{n}\left(\delta_{8,3}\right) & \text { if } n \equiv 1 \quad(\bmod 8) \\ \rho_{n}\left(\delta_{2,1}\right)+\rho_{n}\left(\delta_{4,1}\right)-2 \rho_{n}\left(\delta_{8,1}\right)+\rho_{n}\left(\delta_{8,3}\right) & \text { if } n \equiv 3 \quad(\bmod 8) \\ 3 \rho_{n}\left(\delta_{2,1}\right)-3 \rho_{n}\left(\delta_{4,1}\right)+2 \rho_{n}\left(\delta_{8,2}\right)-\rho_{n}\left(\delta_{8,3}\right) & \text { if } n \equiv 5 \quad(\bmod 8) \\ 5 \rho_{n}\left(\delta_{2,1}\right)+5 \rho_{n}\left(\delta_{4,1}\right)+2 \rho_{n}\left(\delta_{8,1}\right)+\rho_{n}\left(\delta_{8,3}\right) & \text { if } n \equiv 7 \quad(\bmod 8)\end{array}\right.$ 
One can check that the roots $\delta_{8,3}$ are $\sqrt{2} \omega_{40}^{3}, \sqrt{2} \omega_{40}^{11}, \sqrt{2} \omega_{40}^{13}, \sqrt{2} \omega_{40}^{19}, \sqrt{2} \omega_{40}^{21}, \sqrt{2} \omega_{40}^{27}, \sqrt{2} \omega_{40}^{29}, \sqrt{2} \omega_{40}^{37}$, where

$$
\omega_{40}=\frac{1}{\sqrt{2}}\left(\frac{1+\sqrt{5}}{4}-\frac{1-\sqrt{5}}{8} \sqrt{10+2 \sqrt{5}}\right)+\frac{1}{\sqrt{2}}\left(\frac{1+\sqrt{5}}{4}+\frac{1-\sqrt{5}}{8} \sqrt{10+2 \sqrt{5}}\right) i
$$

is the primitive 40-th root of unity with smallest (positive) argument. Due to the presence of the roots of either $\delta_{8,1}$ or $\delta_{8,2}$ in each formula, they can not be periodic in $n$.

An alternative parameterisation. We now present an alternative parameterisation of Eq. (43) which although requires the same number of variables to linearise, is perhaps more natural given the form of $T_{2}\left(a_{0}^{2}+a_{0}+r_{0}\right)$ and $T_{3}\left(a_{0}^{2}+a_{0}+r_{0}\right)$, see $\S 4.3 .2$. In particular, using Lemma 10 part (2) three times with $(\alpha, \beta)=\left(a_{0}^{5}, a_{0}^{3}\right),\left(a_{0}^{5}, a_{0}\right)$ and $\left(a_{0}^{3}, a_{0}\right)$, we have

$$
\begin{aligned}
T_{5}\left(a_{0}^{2}+a_{0}+r_{0}\right) & =T_{1}\left(a_{0}^{5}\right) T_{1}\left(a_{0}^{3}\right)+T_{1}\left(a_{0}^{5}\right) T_{1}\left(a_{0}\right)+T_{1}\left(a_{0}^{3}\right) T_{1}\left(a_{0}\right)+r_{0}\left(T_{2}\left(a_{0}^{3}\right)+T_{2}\left(a_{0}\right)+T_{1}\left(a_{0}^{3}\right) T_{1}\left(a_{0}\right)\right) \\
& +T_{1}\left(a_{0}^{9}+a_{0}^{3}+a_{0}+r_{0}\left(a_{0}^{7}+\left(a_{0}^{5}+a_{0}\right)\left(\begin{array}{l}
n \\
2
\end{array}\right)+\left(a_{0}^{3}+a_{0}\right)\left(\begin{array}{l}
n \\
3
\end{array}\right)+\left(\begin{array}{l}
n \\
5
\end{array}\right)\right)\right) \\
& =T_{2}\left(a_{0}^{5}+a_{0}^{3}\right)+T_{2}\left(a_{0}^{5}+a_{0}\right)+\left(r_{0}+1\right) T_{2}\left(a_{0}^{3}+a_{0}\right) \\
& +T_{1}\left(a_{0}^{9}+a_{0}+r_{0}\left(a_{0}^{7}+a_{0}+\left(a_{0}^{5}+a_{0}\right)\left(\begin{array}{l}
n \\
2
\end{array}\right)+\left(a_{0}^{3}+a_{0}\right)\left(\begin{array}{l}
n \\
3
\end{array}\right)+\left(\begin{array}{l}
n \\
5
\end{array}\right)\right)\right)
\end{aligned}
$$

This can be linearised using the substitutions $a_{0}^{3}+a_{0}=a_{1}^{2}+a_{1}+r_{1}$ and $a_{0}^{5}+a_{0}=a_{2}^{2}+a_{2}+r_{2}$, where $r_{1}$ and $r_{2}$ are the traces of $a_{0}^{3}+a_{0}$ and $a_{0}^{5}+a_{0}$ respectively, which when added together imply that $a_{0}^{5}+a_{0}^{3}=\left(a_{1}+a_{2}\right)^{2}+\left(a_{1}+a_{2}\right)+\left(r_{1}+r_{2}\right)$. This results in

$$
\begin{aligned}
T_{5}\left(a_{0}^{2}+a_{0}+r_{0}\right) & =T_{1}\left(a_{1}^{2} a_{2}+a_{1} a_{2}^{2}+a_{0}^{9}+a_{0}\right. \\
& \left.+r_{0}\left(a_{1}^{3}+a_{1}+a_{0}^{7}+a_{0}+\left(a_{0}^{5}+a_{0}+r_{1}\right)\left(\begin{array}{l}
n \\
2
\end{array}\right)+\left(a_{0}^{3}+a_{0}\right)\left(\begin{array}{l}
n \\
3
\end{array}\right)+\left(\begin{array}{l}
n \\
5
\end{array}\right)\right)\right) .
\end{aligned}
$$

For $16 \leq i \leq 31$ let $\mathbf{i}=\left(i_{4}, i_{3}, i_{2}, i_{1}, i_{0}\right)$. The curves we are interested in are given by the following intersections:

$$
\begin{aligned}
a_{3}^{2}+a_{3} & =i_{4}\left(a_{1}^{2} a_{2}+a_{1} a_{2}^{2}+a_{0}^{9}+a_{0}+r_{0}\left(a_{1}^{3}+a_{1}+a_{0}^{7}+a_{0}+\left(a_{0}^{5}+a_{0}+r_{1}\right)\left(\begin{array}{l}
n \\
2
\end{array}\right)+\left(a_{0}^{3}+a_{0}\right)\left(\begin{array}{l}
n \\
3
\end{array}\right)+\left(\begin{array}{l}
n \\
5
\end{array}\right)\right)\right) \\
& +i_{3}\left(a_{1}^{3}+a_{1}+a_{0}^{7}+a_{0}^{5}+a_{0}^{3}+a_{0}+r_{0}\left(a_{0}^{3}+a_{0}+\left(a_{0}^{3}+a_{0}\right)\left(\begin{array}{l}
n \\
2
\end{array}\right)+\left(\begin{array}{l}
n \\
4
\end{array}\right)\right)+r_{1}\left(\begin{array}{l}
n \\
2
\end{array}\right)\right) \\
& +i_{2}\left(a_{0}^{5}+a_{0}+r_{0}\left(a_{0}^{3}+a_{0}+\left(\begin{array}{l}
n \\
3
\end{array}\right)\right)\right)+i_{1}\left(a_{0}^{3}+a_{0}+r_{0}\left(\begin{array}{l}
n \\
2
\end{array}\right)\right)+i_{0} r_{0}, \\
a_{0}^{3}+a_{0} & =a_{1}^{2}+a_{1}+r_{1} \\
a_{0}^{5}+a_{0} & =a_{2}^{2}+a_{2}+r_{2} .
\end{aligned}
$$

For $i_{4}=1$ the genus of all of these absolutely irreducible curves is 21 - rather than 18 as in the first parameterisation. Nevertheless, once the transform (24) is applied one again obtains the formulae given in Theorem 11.

4.3.2 Some examples from the direct method. For $n$ odd, using Equations (32) to (34) and the alternative parametrisations (39) and (45), one can write down curves arising from the direct method for 
$F_{2}\left(n, t_{1}, t_{2}, t_{3}, t_{4}, t_{5}\right)$, as in $\S 4.2 .2$. Here we give the two simplest examples, valid for all $n \geq 5$.

$$
\begin{aligned}
& F_{2}(n, 0,0,0,0,0)=\#\left\{a \in \mathbb{F}_{2^{n}} \mid T_{1}(a)=0, T_{2}(a)=0, T_{3}(a)=0, T_{4}(a)=0, T_{5}(a)=0\right\} \\
& =\frac{1}{2} \#\left\{a_{0} \in \mathbb{F}_{2^{n}} \mid T_{2}\left(a_{0}^{2}+a_{0}\right)=0, T_{3}\left(a_{0}^{2}+a_{0}\right)=0, T_{4}\left(a_{0}^{2}+a_{0}\right)=0, T_{5}\left(a_{0}^{2}+a_{0}\right)=0\right\} \\
& =\frac{1}{2} \#\left\{a_{0} \in \mathbb{F}_{2^{n}} \mid T_{1}\left(a_{0}^{3}+a_{0}\right)=0, T_{1}\left(a_{0}^{5}+a_{0}\right)=0,\right. \\
& T_{2}\left(a_{0}^{3}+a_{0}\right)+T_{1}\left(a_{0}^{7}+a_{0}^{5}+a_{0}^{3}+a_{0}\right)=0, \\
& \left.T_{1}\left(a_{0}^{5}\right) T_{1}\left(a_{0}^{3}\right)+T_{1}\left(a_{0}^{5}\right) T_{1}\left(a_{0}\right)+T_{1}\left(a_{0}^{3}\right) T_{1}\left(a_{0}\right)+T_{1}\left(a_{0}^{9}+a_{0}^{3}+a_{0}\right)=0\right\} \\
& =\frac{1}{2} \#\left\{a_{0} \in \mathbb{F}_{2^{n}} \mid T_{1}\left(a_{0}^{3}+a_{0}\right)=0, T_{1}\left(a_{0}^{5}+a_{0}\right)=0\right. \text {, } \\
& T_{2}\left(a_{0}^{3}+a_{0}\right)+T_{1}\left(a_{0}^{7}+a_{0}^{5}+a_{0}^{3}+a_{0}\right)=0, \\
& \left.T_{2}\left(a_{0}^{5}+a_{0}^{3}\right)+T_{2}\left(a_{0}^{5}+a_{0}\right)+T_{2}\left(a_{0}^{3}+a_{0}\right)+T_{1}\left(a_{0}^{9}+a_{0}\right)=0\right\} \\
& =\frac{1}{8} \#\left\{\left(a_{0}, a_{1}, a_{2}\right) \in\left(\mathbb{F}_{2^{n}}\right)^{3} \mid a_{1}^{2}+a_{1}=a_{0}^{3}+a_{0}, a_{2}^{2}+a_{2}=a_{0}^{5}+a_{0}\right. \text {, } \\
& T_{1}\left(a_{1}^{3}+a_{1}+a_{0}^{7}+a_{0}^{5}+a_{0}^{3}+a_{0}\right)=0, \\
& \left.T_{2}\left(\left(a_{1}+a_{2}\right)^{2}+\left(a_{1}+a_{2}\right)\right)+T_{1}\left(a_{2}^{3}+a_{2}\right)+T_{1}\left(a_{1}^{3}+a_{1}\right)+T_{1}\left(a_{0}^{9}+a_{0}\right)=0\right\} \\
& =\frac{1}{32} \#\left\{\left(a_{0}, a_{1}, a_{2}, a_{3}, a_{4}\right) \in\left(\mathbb{F}_{2^{n}}\right)^{5} \mid a_{1}^{2}+a_{1}=a_{0}^{3}+a_{0}, a_{2}^{2}+a_{2}=a_{0}^{5}+a_{0}\right. \text {, } \\
& \left.a_{3}^{2}+a_{3}=a_{1}^{3}+a_{1}+a_{0}^{7}+a_{0}^{5}+a_{0}^{3}+a_{0}, a_{4}^{2}+a_{4}=a_{1}^{2} a_{2}+a_{1} a_{2}^{2}+a_{0}^{9}+a_{0}\right\} .
\end{aligned}
$$

The genus of the absolutely irreducible curve this intersection describes is 49 , and thus one can not easily brute-force compute the characteristic polynomial of Frobenius by computing the number of points over $\mathbb{F}_{2^{n}}$ for $n \leq 49$, as for the four coefficient direct method cases. The state-of-the-art $p$-adic point counting algorithms of Tuitman $[49,50]$ are unfortunately not easily adaptable to such intersections, while the prime 2 is problematic. However, by ignoring the fourth trace, letting $\delta_{2,3}(X)=X^{2}-2 X+2$ and $\delta_{4,2}=X^{4}+2 X^{2}+4$, we have

$$
\begin{aligned}
F_{2}(n, 0,0,0, *, 0)= & \#\left\{a \in \mathbb{F}_{2^{n}} \mid T_{1}(a)=0, T_{2}(a)=0, T_{3}(a)=0, T_{5}(a)=0\right\} \\
= & \frac{1}{2} \#\left\{a_{0} \in \mathbb{F}_{2^{n}} \mid T_{2}\left(a_{0}^{2}+a_{0}\right)=0, T_{3}\left(a_{0}^{2}+a_{0}\right)=0, T_{5}\left(a_{0}^{2}+a_{0}\right)=0\right\} \\
= & \frac{1}{2} \#\left\{a_{0} \in \mathbb{F}_{2^{n}} \mid T_{1}\left(a_{0}^{3}+a_{0}\right)=0, T_{1}\left(a_{0}^{5}+a_{0}\right)=0,\right. \\
& \left.\quad T_{2}\left(a_{0}^{5}+a_{0}^{3}\right)+T_{2}\left(a_{0}^{5}+a_{0}\right)+T_{2}\left(a_{0}^{3}+a_{0}\right)+T_{1}\left(a_{0}^{9}+a_{0}\right)=0\right\} \\
= & \frac{1}{16} \#\left\{\left(a_{0}, a_{1}, a_{2}, a_{3}\right) \in\left(\mathbb{F}_{2^{n}}\right)^{4} \mid a_{1}^{2}+a_{1}=a_{0}^{3}+a_{0}, a_{2}^{2}+a_{2}=a_{0}^{5}+a_{0},\right. \\
& \left.\quad a_{3}^{2}+a_{3}=a_{1}^{2} a_{2}+a_{1} a_{2}^{2}+a_{0}^{9}+a_{0}\right\} \\
= & 2^{n-4}-\frac{1}{16}\left(5 \rho_{n}\left(\delta_{2,1}\right)+2 \rho_{n}\left(\delta_{2,2}\right)+2 \rho_{n}\left(\delta_{2,3}\right)+3 \rho_{n}\left(\delta_{4,1}\right)+\rho_{n}\left(\delta_{4,2}\right)+\rho_{n}\left(\delta_{8,3}\right)\right),
\end{aligned}
$$

where in the final equality we have used Magma to compute the characteristic polynomial of Frobenius of the genus 21 curve described by the intersection. Since neither $\delta_{8,1}$ nor $\delta_{8,2}$ feature in this formula, we deduce that the set of formulae for $F_{2}(n, 0,0,0, *, 0)$ are periodic in $n$ with period $\operatorname{LCM}(8,8,4,24,6,40)=120$, in similarity with the two and three coefficient cases for which the periods are 8 and 24 in $n$, respectively.

4.3.3 Further general formulae. While one can not easily compute the zeta function for $F_{2}(n, 0,0,0,0,0)$ using the direct method, one can use the approach of $\S 4.2 .4$ and the alternative parameterisations (39) and (45) to compute $F_{2}\left(n, 0,0,0, t_{4}, t_{5}\right)$ for all $n \geq 5$, as follows.

The conditions $T_{1}(a)=T_{2}(a)=T_{3}(a)=0$ imply that we should set $A=\left\{\left(a_{0}, a_{1}, a_{2}\right) \in\left(\mathbb{F}_{2^{n}}\right)^{3} \mid\right.$ $\left.a_{0}^{3}+a_{0}=a_{1}^{2}+a_{1} a_{0}^{5}+a_{0}=a_{2}^{2}+a_{2}\right\}$. Furthermore let $\mathbf{f}=\left(T_{5}\left(a_{0}^{2}+a_{0}\right), T_{4}\left(a_{0}^{2}+a_{0}\right)\right)=\left(T_{1}\left(a_{1}^{2} a_{2}+a_{1} a_{2}^{2}+\right.\right.$ 
$\left.\left.a_{0}^{9}+a_{0}\right), T_{1}\left(a_{1}^{3}+a_{1}+a_{0}^{7}+a_{0}^{5}+a_{0}^{3}+a_{0}\right)\right)$. Then by the generalisation of the transform (24) we have

$$
\left[\begin{array}{l}
F_{2}(n, 0,0,0,0,0) \\
F_{2}(n, 0,0,0,1,0) \\
F_{2}(n, 0,0,0,0,1) \\
F_{2}(n, 0,0,0,1,1)
\end{array}\right]=\left[\begin{array}{l}
N(\mathbf{0}) \\
N(\mathbf{1}) \\
N(\mathbf{2}) \\
N(\mathbf{3})
\end{array}\right]=\frac{1}{16}\left[\begin{array}{rrrr}
-1 & 1 & 1 & 1 \\
1 & -1 & 1 & -1 \\
1 & 1 & -1 & -1 \\
1 & -1 & -1 & 1
\end{array}\right]\left[\begin{array}{l}
V(\mathbf{0} \cdot \mathbf{f}) \\
V(\mathbf{1} \cdot \mathbf{f}) \\
V(\mathbf{2} \cdot \mathbf{f}) \\
V(\mathbf{3} \cdot \mathbf{f})
\end{array}\right],
$$

where we have a factor of $1 / 16$ rather than $1 / 2$ because of the three factors of $1 / 2$ arising from the introduction of the variables $a_{0}, a_{1}$ and $a_{2}$ defining $A$. Note that $V(\mathbf{0} \cdot \mathbf{f})=|A|=2^{n}-2 \rho_{n}\left(\delta_{2,1}\right)-\rho_{n}\left(\delta_{2,2}\right)-$ $\rho_{n}\left(\delta_{4,1}\right)$. For $1 \leq i \leq 3$ let $\mathbf{i}=\left(i_{1}, i_{0}\right)$. We thus have:

$$
\begin{aligned}
V(\mathbf{i} \cdot \mathbf{f})=\frac{1}{2} \#\left\{\left(a_{0}, a_{1}, a_{2}, a_{3}\right) \mid a_{0}^{3}+a_{0}=a_{1}^{2}+a_{1}, a_{0}^{5}+a_{0}=a_{2}^{2}+a_{2}\right. \\
\\
\left.\quad a_{3}^{2}+a_{3}=i_{1}\left(a_{1}^{2} a_{2}+a_{1} a_{2}^{2}+a_{0}^{9}+a_{0}\right)+i_{0}\left(a_{1}^{3}+a_{1}+a_{0}^{7}+a_{0}^{5}+a_{0}^{3}+a_{0}\right)\right\} .
\end{aligned}
$$

For $i=1,2$ and 3 these are absolutely irreducible curves of genus 5,17,21 and 21 respectively, and thus their zeta functions are far easier to compute than for those curves arising from the direct method. Let $\delta_{8,4}(X)=X^{8}+2 X^{6}-4 X^{5}+2 X^{4}-8 X^{3}+8 X^{2}+16$. Combining the $V(\mathbf{i} \cdot \mathbf{f})$ as per Eq. (47) gives the following theorem.

Theorem 12. For $n \geq 5$ we have

$$
\begin{aligned}
F_{2}(n, 0,0,0,0,0)=2^{n-5}- & \frac{1}{32}\left(7 \rho_{n}\left(\delta_{2,1}\right)+4 \rho_{n}\left(\delta_{2,2}\right)+2 \rho_{n}\left(\delta_{2,3}\right)+5 \rho_{n}\left(\delta_{4,1}\right)+3 \rho_{n}\left(\delta_{4,2}\right)+2 \rho_{n}\left(\delta_{8,1}\right)\right. \\
& \left.+\rho_{n}\left(\delta_{8,2}\right)+\rho_{n}\left(\delta_{8,3}\right)+\rho_{n}\left(\delta_{8,4}\right)\right), \\
F_{2}(n, 0,0,0,1,0)=2^{n-5}- & \frac{1}{32}\left(3 \rho_{n}\left(\delta_{2,1}\right)+2 \rho_{n}\left(\delta_{2,3}\right)+\rho_{n}\left(\delta_{4,1}\right)-\rho_{n}\left(\delta_{4,2}\right)-2 \rho_{n}\left(\delta_{8,1}\right)-\rho_{n}\left(\delta_{8,2}\right)\right. \\
& \left.+\rho_{n}\left(\delta_{8,3}\right)-\rho_{n}\left(\delta_{8,4}\right)\right), \\
F_{2}(n, 0,0,0,0,1)=2^{n-5}- & \frac{1}{32}\left(\rho_{n}\left(\delta_{2,1}\right)+2 \rho_{n}\left(\delta_{2,2}\right)-2 \rho_{n}\left(\delta_{2,3}\right)-3 \rho_{n}\left(\delta_{4,1}\right)-3 \rho_{n}\left(\delta_{4,2}\right)+\rho_{n}\left(\delta_{8,2}\right)\right. \\
& \left.-\rho_{n}\left(\delta_{8,3}\right)-\rho_{n}\left(\delta_{8,4}\right)\right), \\
F_{2}(n, 0,0,0,1,1)=2^{n-5}- & \frac{1}{32}\left(-3 \rho_{n}\left(\delta_{2,1}\right)-2 \rho_{n}\left(\delta_{2,2}\right)-2 \rho_{n}\left(\delta_{2,3}\right)+\rho_{n}\left(\delta_{4,1}\right)+\rho_{n}\left(\delta_{4,2}\right)-\rho_{n}\left(\delta_{8,2}\right)\right. \\
& \left.-\rho_{n}\left(\delta_{8,3}\right)+\rho_{n}\left(\delta_{8,4}\right)\right) .
\end{aligned}
$$

Note that the formula for $F_{2}(n, 0,0,0,0,0)$ arises from the curve (46) of genus 49. This approach therefore provides a much more efficient way to compute the zeta function than the direct method does. Further note that the terms involving $\delta_{8,1}, \delta_{8,2}$ and $\delta_{8,4}$ all cancel in $F_{2}(n, 0,0,0,0,0)+F_{2}(n, 0,0,0,1,0)=F_{2}(0,0,0, *, 0)$, as well as in $F_{2}(n, 0,0,0,0,1)+F_{2}(n, 0,0,0,1,1)=F_{2}(0,0,0, *, 1)$, which has period 120 in $n$ as well.

\subsection{Computing $F_{2}\left(n, t_{1}, t_{2}, t_{3}, t_{4}, t_{5}, t_{6}\right)$}

In this subsection and the next we shall only detail the indirect method, since the curves produced have genera which are already very large, making the brute force computation of the characteristic polynomials of Frobenius prohibitive, while the direct method produce curves of even larger genus.

Let $\mathbf{f}=\left(T_{6}, T_{5}, T_{4}, T_{3}, T_{2}, T_{1}\right)$. To determine $V(\mathbf{i} \cdot \mathbf{f})$ for $32 \leq i \leq 63$, we use Lemma 10 part (6). In particular, setting $\alpha=a_{0}^{2}$ and $\beta=a_{0}$ for $r_{0}=0$, and $\alpha=a_{0}^{2}+a_{0}$ and $\beta=1$ for $r_{0}=1$, and evaluating mod 2 gives the following:

$$
\begin{aligned}
T_{6}\left(a_{0}^{2}+a_{0}+r_{0}\right) & =T_{3}\left(a_{0}\right)+T_{2}\left(a_{0}^{3}\right) T_{1}\left(a_{0}^{3}\right)+T_{2}\left(a_{0}^{3}\right) T_{1}\left(a_{0}\right)+T_{2}\left(a_{0}\right) T_{1}\left(a_{0}^{3}\right)+T_{2}\left(a_{0}^{5}\right)+T_{2}\left(a_{0}\right) \\
& +T_{1}\left(a_{0}^{7}\right) T_{1}\left(a_{0}^{3}\right)+T_{1}\left(a_{0}^{7}\right) T_{1}\left(a_{0}\right)+T_{1}\left(a_{0}^{5}\right) T_{1}\left(a_{0}^{3}\right)+T_{1}\left(a_{0}^{3}\right) T_{1}\left(a_{0}\right) \\
& +r_{0}\left(T_{2}\left(a_{0}^{3}\right)+T_{2}\left(a_{0}\right)+T_{1}\left(a_{0}^{3}\right) T_{1}\left(a_{0}\right)+T_{1}\left(a_{0}^{7}\right)\right)\left(\begin{array}{c}
n \\
2
\end{array}\right) \\
& +T_{1}\left(a_{0}^{11}+a_{0}^{7}+r_{0}\left(a_{0}^{3}+a_{0}\right)+r_{0}\left(a_{0}^{5}+a_{0}\right)\left(\begin{array}{c}
n \\
3
\end{array}\right)+r_{0}\left(a_{0}^{3}+a_{0}\right)\left(\begin{array}{c}
n \\
4
\end{array}\right)+r_{0}\left(\begin{array}{c}
n \\
6
\end{array}\right)\right) .
\end{aligned}
$$


This can be reduced to a $T_{1}$ expression using the substitutions $a_{0}=a_{1}^{2}+a_{1}+r_{1}, a_{0}^{3}=a_{2}^{2}+a_{2}+r_{2}$ and $a_{0}^{5}=a_{3}^{2}+a_{3}+r_{3}$, where $r_{1}, r_{2}, r_{3} \in \mathbb{F}_{2}$ are the traces of $a_{0}, a_{0}^{3}$ and $a_{0}^{5}$ respectively. This results in

$$
\begin{aligned}
T_{6}\left(a_{0}^{2}+a_{0}+r_{0}\right) & =T_{1}\left(a_{3}^{3}+a_{3}+\left(r_{1}+r_{2}\right)\left(a_{2}^{3}+a_{2}+a_{1}^{3}+a_{1}+a_{0}^{7}\right)+a_{1}^{5}+a_{1}^{3}+a_{0}^{11}+a_{0}^{7}+r_{0} r_{1}+r_{0} r_{2}\right. \\
& +r_{1} r_{2}+r_{2} r_{3}+\left(r_{0}\left(a_{2}^{3}+a_{2}+a_{1}^{3}+a_{1}+a_{0}^{7}+r_{1}+r_{2}+r_{1} r_{2}+1\right)+r_{1}+r_{2}+r_{3}\right)\left(\begin{array}{c}
n \\
2
\end{array}\right) \\
& \left.+\left(r_{0} r_{1}+r_{0} r_{3}+r_{1}\right)\left(\begin{array}{c}
n \\
3
\end{array}\right)+r_{0}\left(r_{1}+r_{2}\right)\left(\begin{array}{c}
n \\
4
\end{array}\right)+r_{0}\left(\begin{array}{c}
n \\
6
\end{array}\right)\right) .
\end{aligned}
$$

For $32 \leq i \leq 63$ let $\mathbf{i}=\left(i_{5}, i_{4}, i_{3}, i_{2}, i_{1}, i_{0}\right)$. The curves we are interested in are given by the following intersections:

$$
\begin{aligned}
a_{4}^{2}+a_{4} & =i_{5}\left(a_{3}^{3}+a_{3}+\left(r_{1}+r_{2}\right)\left(a_{2}^{3}+a_{2}+a_{1}^{3}+a_{1}+a_{0}^{7}\right)+a_{1}^{5}+a_{1}^{3}+a_{0}^{11}+a_{0}^{7}+r_{0} r_{1}+r_{0} r_{2}\right. \\
& +r_{1} r_{2}+r_{2} r_{3}+\left(r_{0}\left(a_{2}^{3}+a_{2}+a_{1}^{3}+a_{1}+a_{0}^{7}+r_{1}+r_{2}+r_{1} r_{2}+1\right)+r_{1}+r_{2}+r_{3}\right)\left(\begin{array}{c}
n \\
2
\end{array}\right) \\
& \left.+\left(r_{0} r_{1}+r_{0} r_{3}+r_{1}\right)\left(\begin{array}{c}
n \\
3
\end{array}\right)+r_{0}\left(r_{1}+r_{2}\right)\left(\begin{array}{c}
n \\
4
\end{array}\right)+r_{0}\left(\begin{array}{c}
n \\
6
\end{array}\right)\right) \\
& +i_{4}\left(r_{0}\left(a_{2}^{3}+a_{2}+a_{1}^{3}+a_{1}\right)+a_{0}^{9}+r_{0} a_{0}^{7}+\left(r_{1}+r_{2}\right) a_{0}^{5}+r_{1}+r_{2}+r_{1} r_{2}+r_{0} r_{1} r_{2}\right. \\
& \left.+\left(r_{0} a_{0}^{5}+r_{0} r_{2}\right)\left(\begin{array}{c}
n \\
2
\end{array}\right)+\left(r_{0} r_{1}+r_{0} r_{2}\right)\left(\begin{array}{c}
n \\
3
\end{array}\right)+r_{0}\left(\begin{array}{c}
n \\
5
\end{array}\right)\right) \\
& +i_{3}\left(a_{2}^{3}+a_{2}+a_{1}^{3}+a_{1}+a_{0}^{7}+a_{0}^{5}+\left(r_{0}+1\right)\left(r_{1}+r_{2}\right)\left(\begin{array}{c}
n \\
2
\end{array}\right)+r_{0} r_{1}+r_{0} r_{2}+r_{1} r_{2}+r_{2}+r_{0}\left(\begin{array}{c}
n \\
4
\end{array}\right)\right) \\
& +i_{2}\left(a_{0}^{5}+a_{0}+r_{0}\left(a_{0}^{3}+a_{0}+\left(\begin{array}{c}
n \\
3
\end{array}\right)\right)\right)+i_{1}\left(a_{0}^{3}+a_{0}+r_{0}\left(\begin{array}{c}
n \\
2
\end{array}\right)\right)+i_{0} r_{0}, \\
a_{0} & =a_{1}^{2}+a_{1}+r_{1}, \\
a_{0}^{3} & =a_{2}^{2}+a_{2}+r_{2} . \\
a_{0}^{5} & =a_{3}^{2}+a_{3}+r_{3} .
\end{aligned}
$$

Corollary 1 implies that mod 2 one has

$$
\left(\left(\begin{array}{l}
n \\
6
\end{array}\right),\left(\begin{array}{l}
n \\
5
\end{array}\right),\left(\begin{array}{l}
n \\
4
\end{array}\right),\left(\begin{array}{l}
n \\
3
\end{array}\right),\left(\begin{array}{l}
n \\
2
\end{array}\right),\left(\begin{array}{l}
n \\
1
\end{array}\right)\right) \equiv\left\{\begin{array}{lll}
(0,0,0,0,0,1) & \text { if } n \equiv 1 & (\bmod 8) \\
(0,0,0,1,1,1) & \text { if } n \equiv 3 & (\bmod 8) \\
(0,1,1,0,0,1) & \text { if } n \equiv 5 & (\bmod 8) \\
(1,1,1,1,1,1) & \text { if } n \equiv 7 & (\bmod 8)
\end{array}\right.
$$

and hence there are four cases to consider when computing the zeta functions of each of the curves (48).

For $i_{5}=1$ the genus of all of the above curves is $50^{\dagger}$ and therefore the brute-force computation of their zeta functions is non-trivial. A curve-specific analysis may yield the zeta functions more efficiently, but since our algorithm is arguably more interesting than the explicit formulae, we leave this as an open problem.

\subsection{Computing $F_{2}\left(n, t_{1}, t_{2}, t_{3}, t_{4}, t_{5}, t_{6}, t_{7}\right)$}

Let $\mathbf{f}=\left(T_{7}, T_{6}, T_{5}, T_{4}, T_{3}, T_{2}, T_{1}\right)$. To determine $V(\mathbf{i} \cdot \mathbf{f})$ for $64 \leq i \leq 127$, we use Lemma 10 part $(7)$. In particular, setting $\alpha=a_{0}^{2}$ and $\beta=a_{0}$ for $r_{0}=0$, and $\alpha=a_{0}^{2}+a_{0}$ and $\beta=1$ for $r_{0}=1$, and evaluating

\footnotetext{
${ }^{\dagger}$ See 6CoefficientsGenus.m.
} 
mod 2 gives the following:

$$
\begin{aligned}
T_{7}\left(a_{0}^{2}+a_{0}+r_{0}\right) & =r_{0} T_{3}\left(a_{0}\right)+T_{1}\left(a_{0}^{5}\right) T_{1}\left(a_{0}^{3}\right) T_{1}\left(a_{0}\right)+r_{0}\left(T_{2}\left(a_{0}^{3}\right) T_{1}\left(a_{0}^{3}\right)+T_{2}\left(a_{0}^{3}\right) T_{1}\left(a_{0}\right)+T_{2}\left(a_{0}\right) T_{1}\left(a_{0}^{3}\right)\right) \\
& +T_{2}\left(a_{0}^{3}\right) T_{1}\left(a_{0}^{5}\right)+T_{2}\left(a_{0}^{3}\right) T_{1}\left(a_{0}\right)+T_{2}\left(a_{0}\right) T_{1}\left(a_{0}^{5}\right)+T_{2}\left(a_{0}\right) T_{1}\left(a_{0}\right)+r_{0}\left(T_{2}\left(a_{0}\right)\right. \\
& \left.+T_{2}\left(a_{0}^{3}\right)\right)\left(\begin{array}{c}
n \\
3
\end{array}\right)+r_{0} T_{2}\left(a_{0}\right)+r_{0} T_{2}\left(a_{0}^{5}\right)+T_{1}\left(a_{0}^{9}\right) T_{1}\left(a_{0}^{3}\right)+T_{1}\left(a_{0}^{9}\right) T_{1}\left(a_{0}\right)+T_{1}\left(a_{0}^{7}\right) T_{1}\left(a_{0}^{5}\right) \\
& +r_{0} T_{1}\left(a_{0}^{7}\right) T_{1}\left(a_{0}^{3}\right)+\left(r_{0}+1\right) T_{1}\left(a_{0}^{7}\right) T_{1}\left(a_{0}\right)+\left(r_{0}+1+r_{0}\left(\begin{array}{c}
n \\
2
\end{array}\right)\right) T_{1}\left(a_{0}^{5}\right) T_{1}\left(a_{0}^{3}\right) \\
& +\left(1+r_{0}\left(\begin{array}{l}
n \\
2
\end{array}\right)\right) T_{1}\left(a_{0}^{5}\right) T_{1}\left(a_{0}\right)+\left(r_{0}+1+r_{0}\left(\begin{array}{c}
n \\
2
\end{array}\right)+r_{0}\left(\begin{array}{c}
n \\
3
\end{array}\right)\right) T_{1}\left(a_{0}^{3}\right) T_{1}\left(a_{0}\right) \\
& +T_{1}\left(a_{0}^{13}+\left(r_{0}+1\right) a_{0}^{11}+a_{0}^{9}+r_{0}\left(a_{0}^{7}+a_{0}^{5}+a_{0}^{3}\right)+a_{0}+r_{0}\left(a_{0}^{9}+a_{0}^{3}+a_{0}\right)\left(\begin{array}{c}
n \\
2
\end{array}\right)\right. \\
& \left.+r_{0} a_{0}^{7}\left(\begin{array}{c}
n \\
3
\end{array}\right)+r_{0}\left(a_{0}^{5}+a_{0}\right)\left(\begin{array}{c}
n \\
4
\end{array}\right)+r_{0}\left(a_{0}^{3}+a_{0}\right)\left(\begin{array}{c}
n \\
5
\end{array}\right)+r_{0}\left(\begin{array}{c}
n \\
7
\end{array}\right)\right) .
\end{aligned}
$$

As in the six coefficient case, this can be reduced to a $T_{1}$ expression using the substitutions $a_{0}=a_{1}^{2}+a_{1}+r_{1}$, $a_{0}^{3}=a_{2}^{2}+a_{2}+r_{2}$ and $a_{0}^{5}=a_{3}^{2}+a_{3}+r_{3}$, where $r_{1}, r_{2}, r_{3} \in \mathbb{F}_{2}$ are the traces of $a_{0}, a_{0}^{3}$ and $a_{0}^{5}$ respectively. This results in

$$
\begin{aligned}
T_{7}\left(a_{0}^{2}+a_{0}+r_{0}\right) & =T_{1}\left(r_{0}\left(a_{3}^{3}+a_{3}\right)+\left(r_{0} r_{1}+r_{0} r_{2}+r_{1}+r_{3}+r_{0}\left(\begin{array}{l}
n \\
3
\end{array}\right)\right)\left(a_{2}^{3}+a_{2}\right)+r_{0}\left(a_{1}^{5}+a_{1}^{3}\right)\right. \\
& +\left(r_{0} r_{1}+r_{0} r_{2}+r_{1}+r_{3}+r_{0}\left(\begin{array}{l}
n \\
3
\end{array}\right)\right)\left(a_{1}^{3}+a_{1}\right)+a_{0}^{13}+\left(r_{0}+1\right) a_{0}^{11} \\
& +\left(r_{1}+r_{2}+1+r_{0}\left(\begin{array}{l}
n \\
2
\end{array}\right)\right) a_{0}^{9}+\left(r_{0}+r_{1}+r_{3}+r_{0} r_{1}+r_{0} r_{2}+r_{0}\left(\begin{array}{c}
n \\
3
\end{array}\right)\right) a_{0}^{7} \\
& +r_{1}+r_{0} r_{2}+r_{0} r_{3}+r_{1} r_{2}+r_{1} r_{3}+r_{2} r_{3}+r_{0} r_{1} r_{2}+r_{0} r_{2} r_{3}+r_{1} r_{2} r_{3} \\
& +\left(r_{1}+r_{0} r_{3}+r_{1} r_{2}+r_{1} r_{3}+r_{2} r_{3}+r_{0} r_{1} r_{2}+r_{0} r_{1} r_{3}+r_{0} r_{2} r_{3}\right)\left(\begin{array}{c}
n \\
2
\end{array}\right) \\
& +\left(r_{0} r_{1}+r_{0} r_{1} r_{2}\right)\left(\begin{array}{l}
n \\
3
\end{array}\right)+\left(r_{0} r_{1}+r_{0} r_{2}\right)\left(\begin{array}{c}
n \\
2
\end{array}\right)\left(\begin{array}{c}
n \\
3
\end{array}\right)+\left(r_{0} r_{1}+r_{0} r_{3}\right)\left(\begin{array}{c}
n \\
4
\end{array}\right) \\
& \left.+\left(r_{0} r_{1}+r_{0} r_{2}\right)\left(\begin{array}{l}
n \\
5
\end{array}\right)+r_{0}\left(\begin{array}{l}
n \\
7
\end{array}\right)\right) .
\end{aligned}
$$

For $64 \leq i \leq 127$ let $\mathbf{i}=\left(i_{6}, i_{5}, i_{4}, i_{3}, i_{2}, i_{1}, i_{0}\right)$. Applying Transform 3 , the curves we are interested in are given by the following intersections:

$$
\begin{aligned}
a_{4}^{2}+a_{4} & =i_{6}\left(r_{0}\left(a_{3}^{3}+a_{3}\right)+\left(r_{0} r_{1}+r_{0} r_{2}+r_{1}+r_{3}+r_{0}\left(\begin{array}{c}
n \\
3
\end{array}\right)\right)\left(a_{2}^{3}+a_{2}\right)+r_{0}\left(a_{1}^{5}+a_{1}^{3}\right)\right. \\
& +\left(r_{0} r_{1}+r_{0} r_{2}+r_{1}+r_{3}+r_{0}\left(\begin{array}{c}
n \\
3
\end{array}\right)\right)\left(a_{1}^{3}+a_{1}\right)+a_{0}^{13}+\left(r_{0}+1\right) a_{0}^{11} \\
& +\left(r_{1}+r_{2}+1+r_{0}\left(\begin{array}{c}
n \\
2
\end{array}\right)\right) a_{0}^{9}+\left(r_{0}+r_{1}+r_{3}+r_{0} r_{1}+r_{0} r_{2}+r_{0}\left(\begin{array}{l}
n \\
3
\end{array}\right)\right) a_{0}^{7} \\
& +r_{1}+r_{0} r_{2}+r_{0} r_{3}+r_{1} r_{2}+r_{1} r_{3}+r_{2} r_{3}+r_{0} r_{1} r_{2}+r_{0} r_{2} r_{3}+r_{1} r_{2} r_{3} \\
& +\left(r_{1}+r_{0} r_{3}+r_{1} r_{2}+r_{1} r_{3}+r_{2} r_{3}+r_{0} r_{1} r_{2}+r_{0} r_{1} r_{3}+r_{0} r_{2} r_{3}\right)\left(\begin{array}{c}
n \\
2
\end{array}\right) \\
& +\left(r_{0} r_{1}+r_{0} r_{1} r_{2}\right)\left(\begin{array}{c}
n \\
3
\end{array}\right)+\left(r_{0} r_{1}+r_{0} r_{2}\right)\left(\begin{array}{c}
n \\
2
\end{array}\right)\left(\begin{array}{l}
n \\
3
\end{array}\right)+\left(r_{0} r_{1}+r_{0} r_{3}\right)\left(\begin{array}{l}
n \\
4
\end{array}\right)
\end{aligned}
$$




$$
\begin{aligned}
& \left.+\left(r_{0} r_{1}+r_{0} r_{2}\right)\left(\begin{array}{l}
n \\
5
\end{array}\right)+r_{0}\left(\begin{array}{l}
n \\
7
\end{array}\right)\right) \\
& +i_{5}\left(a_{3}^{3}+a_{3}+\left(r_{1}+r_{2}\right)\left(a_{2}^{3}+a_{2}+a_{1}^{3}+a_{1}+a_{0}^{7}\right)+a_{1}^{5}+a_{1}^{3}+a_{0}^{11}+a_{0}^{7}+r_{0} r_{1}+r_{0} r_{2}\right. \\
& +r_{1} r_{2}+r_{2} r_{3}+\left(r_{0}\left(a_{2}^{3}+a_{2}+a_{1}^{3}+a_{1}+a_{0}^{7}+r_{1}+r_{2}+r_{1} r_{2}+1\right)+r_{1}+r_{2}+r_{3}\right)\left(\begin{array}{c}
n \\
2
\end{array}\right) \\
& \left.+\left(r_{0} r_{1}+r_{0} r_{3}+r_{1}\right)\left(\begin{array}{l}
n \\
3
\end{array}\right)+r_{0}\left(r_{1}+r_{2}\right)\left(\begin{array}{l}
n \\
4
\end{array}\right)+r_{0}\left(\begin{array}{l}
n \\
6
\end{array}\right)\right) \\
& +i_{4}\left(r_{0}\left(a_{2}^{3}+a_{2}+a_{1}^{3}+a_{1}\right)+a_{0}^{9}+r_{0} a_{0}^{7}+\left(r_{1}+r_{2}\right) a_{0}^{5}+r_{1}+r_{2}+r_{1} r_{2}+r_{0} r_{1} r_{2}\right. \\
& \left.+\left(r_{0} a_{0}^{5}+r_{0} r_{2}\right)\left(\begin{array}{l}
n \\
2
\end{array}\right)+\left(r_{0} r_{1}+r_{0} r_{2}\right)\left(\begin{array}{l}
n \\
3
\end{array}\right)+r_{0}\left(\begin{array}{l}
n \\
5
\end{array}\right)\right) \\
& +i_{3}\left(a_{2}^{3}+a_{2}+a_{1}^{3}+a_{1}+a_{0}^{7}+a_{0}^{5}+\left(r_{0}+1\right)\left(r_{1}+r_{2}\right)\left(\begin{array}{c}
n \\
2
\end{array}\right)+r_{0} r_{1}+r_{0} r_{2}+r_{1} r_{2}+r_{2}+r_{0}\left(\begin{array}{c}
n \\
4
\end{array}\right)\right) \\
& +i_{2}\left(a_{0}^{5}+a_{0}+r_{0}\left(a_{0}^{3}+a_{0}+\left(\begin{array}{c}
n \\
3
\end{array}\right)\right)\right)+i_{1}\left(a_{0}^{3}+a_{0}+r_{0}\left(\begin{array}{c}
n \\
2
\end{array}\right)\right)+i_{0} r_{0}, \\
& a_{0}=a_{1}^{2}+a_{1}+r_{1}, \\
& a_{0}^{3}=a_{2}^{2}+a_{2}+r_{2} . \\
& a_{0}^{5}=a_{3}^{2}+a_{3}+r_{3} .
\end{aligned}
$$

Corollary 1 implies that mod 2 one has

$$
\left(\left(\begin{array}{l}
n \\
7
\end{array}\right),\left(\begin{array}{l}
n \\
6
\end{array}\right),\left(\begin{array}{l}
n \\
5
\end{array}\right),\left(\begin{array}{l}
n \\
4
\end{array}\right),\left(\begin{array}{l}
n \\
3
\end{array}\right),\left(\begin{array}{l}
n \\
2
\end{array}\right),\left(\begin{array}{l}
n \\
1
\end{array}\right)\right) \equiv\left\{\begin{array}{lll}
(0,0,0,0,0,0,1) & \text { if } n \equiv 1 \quad(\bmod 8) \\
(0,0,0,0,1,1,1) & \text { if } n \equiv 3 \quad(\bmod 8) \\
(0,0,1,1,0,0,1) & \text { if } n \equiv 5 \quad(\bmod 8) \\
(1,1,1,1,1,1,1) & \text { if } n \equiv 7 & (\bmod 8)
\end{array}\right.
$$

and hence there are four cases to consider when computing the zeta functions of each of the curves (49).

For $i_{6}=1$, the genus of each of the above curves is $58^{\dagger}$. Therefore, we again leave it as an open problem to determine their zeta functions.

\subsection{Reducing the prescribed coefficients problem to the prescribed traces problem}

In this section we give formulae for all $I_{2}\left(n, t_{1}, t_{2}, t_{3}, t_{4}\right)$ in terms of the $F_{2}\left(n, t_{1}, t_{2}, t_{3}, t_{4}\right)$. In the work [30], Koma and Panario gave explicit formulae for the transform between $I_{2}\left(n, t_{1}, \ldots, t_{l}\right)$ and $F_{2}\left(n, t_{1}, \ldots, t_{l}\right)$ for $l=4$ and $l=5$; the extension to the $l=6$ and $l=7$ cases was also explained and a sketch of how to compute the transform for arbitrary $l \geq 8$ was provided. However, there is a small error in the application of the multinomial theorem which unfortunately invalidates several lemmas, propositions and some of the formulae. Since in this work we have provided formulae for $F_{2}\left(n, t_{1}, t_{2}, t_{3}, t_{4}\right)$ we now provide the correct formulae for $I_{2}\left(n, t_{1}, t_{2}, t_{3}, t_{4}\right)$. The following lemma is simply a subcase of Lemma 6 .

Lemma 12. For each integer $d \geq 1$ and $f(x) \in \mathbb{F}_{2}[x]$,

1. $T_{1}\left(f^{d}\right)=d T_{1}(f)$

2. $T_{2}\left(f^{d}\right)=\left(\begin{array}{l}d \\ 2\end{array}\right) T_{1}(f)+d T_{2}(f)$

3. $T_{3}\left(f^{d}\right)=\left(\begin{array}{l}d \\ 3\end{array}\right) T_{1}(f)+d T_{3}(f)$

4. $T_{4}\left(f^{d}\right)=\left(\begin{array}{l}d \\ 4\end{array}\right) T_{1}(f)+\left(\begin{array}{l}d \\ 2\end{array}\right) T_{2}(f)+d T_{4}(f)+(d-2)\left(\begin{array}{l}d \\ 2\end{array}\right) T_{1}(f) T_{2}(f)$

\footnotetext{
${ }^{\dagger}$ See 7CoefficientsGenus.m.
} 
It is the final term of Lemma 12 part (4) that is missing from [30, Prop. 2.1], which has coefficient 1 for $d \equiv 3(\bmod 4)$.

Recall from Corollary 1 that the featured binomial coefficients have maximal period 8 and hence we need to consider the residue classes of $d \bmod 8$. For brevity we write $d \equiv a(8)$ to denote $d \equiv a(\bmod 8)$ and similarly write $d \equiv a(4)$ to denote $d \equiv a(\bmod 4)$. As in [8] let $\operatorname{Irr}(n)$ denote the set of all irreducible polynomials of degree $n$ over $\mathbb{F}_{2}$, and let $a \cdot \operatorname{Irr}(n)$ denote the multiset consisting of $a$ copies of $\operatorname{Irr}(n)$. Following [8] and [57], applying Lemma 12 gives the following result.

Lemma 13. For $n \geq 4$ we have

$$
\begin{aligned}
& F_{2}\left(n, t_{1}, t_{2}, t_{3}, t_{4}\right)=\left|\bigcup_{\beta \in \mathbb{F}_{2^{n}}, T_{1}(\beta)=t_{1}, T_{2}(\beta)=t_{2}, T_{3}(\beta)=t_{3}, T_{4}(\beta)=t_{4}} \operatorname{Min}(\beta)\right| \\
& =\mid \bigcup_{d \mid n} \frac{n}{d}\left\{f \in \operatorname{Irr}\left(\frac{n}{d}\right): d T_{1}(f)=t_{1},\left(\begin{array}{l}
d \\
2
\end{array}\right) T_{1}(f)+d T_{2}(f)=t_{2},\left(\begin{array}{l}
d \\
3
\end{array}\right) T_{1}(f)+d T_{3}(f)=t_{3}\right. \\
& \left.\left(\begin{array}{l}
d \\
4
\end{array}\right) T_{1}(f)+\left(\begin{array}{l}
d \\
2
\end{array}\right) T_{2}(f)+d T_{4}(f)+(d-2)\left(\begin{array}{l}
d \\
2
\end{array}\right) T_{1}(f) T_{2}(f)=t_{4}\right\} \mid \\
& =\left|\bigcup_{d \mid n, d \equiv 0(8)} \frac{n}{d}\left\{f \in \operatorname{Irr}\left(\frac{n}{d}\right): 0=t_{1}, 0=t_{2}, 0=t_{3}, 0=t_{4}\right\}\right| \\
& +\left|\bigcup_{d \mid n, d \equiv 1(8)} \frac{n}{d}\left\{f \in \operatorname{Irr}\left(\frac{n}{d}\right): T_{1}(f)=t_{1}, T_{2}(f)=t_{2}, T_{3}(f)=t_{3}, T_{4}(f)=t_{4}\right\}\right| \\
& +\left|\bigcup_{d \mid n, d \equiv 2(8)} \frac{n}{d}\left\{f \in \operatorname{Irr}\left(\frac{n}{d}\right): 0=t_{1}, T_{1}(f)=t_{2}, 0=t_{3}, T_{2}(f)=t_{4}\right\}\right| \\
& +\mid \bigcup_{d \mid n, d \equiv 3(8)} \frac{n}{d}\left\{f \in \operatorname{Irr}\left(\frac{n}{d}\right): T_{1}(f)=t_{1}, T_{1}(f)+T_{2}(f)=t_{2}, T_{1}(f)+T_{3}(f)=t_{3},\right. \\
& \left.T_{2}(f)+T_{4}(f)+T_{1}(f) T_{2}(f)=t_{4}\right\} \\
& +\left|\bigcup_{d \mid n, d \equiv 4(8)} \frac{n}{d}\left\{f \in \operatorname{Irr}\left(\frac{n}{d}\right): 0=t_{1}, 0=t_{2}, 0=t_{3}, T_{1}(f)=t_{4}\right\}\right| \\
& +\left|\bigcup_{d \mid n, d \equiv 5(8)} \frac{n}{d}\left\{f \in \operatorname{Irr}\left(\frac{n}{d}\right): T_{1}(f)=t_{1}, T_{2}(f)=t_{2}, T_{3}(f)=t_{3}, T_{1}(f)+T_{4}(f)=t_{4}\right\}\right| \\
& +\left|\bigcup_{d \mid n, d \equiv 6(8)} \frac{n}{d}\left\{f \in \operatorname{Irr}\left(\frac{n}{d}\right): 0=t_{1}, T_{1}(f)=t_{2}, 0=t_{3}, T_{1}(f)+T_{2}(f)=t_{4}\right\}\right| \\
& +\mid \bigcup_{d \mid n, d \equiv 7(8)} \frac{n}{d}\left\{f \in \operatorname{Irr}\left(\frac{n}{d}\right): T_{1}(f)=t_{1}, T_{1}(f)+T_{2}(f)=t_{2}, T_{1}(f)+T_{3}(f)=t_{3},\right. \\
& \left.T_{1}(f)+T_{2}(f)+T_{4}(f)+T_{1}(f) T_{2}(f)=t_{4}\right\} \mid .
\end{aligned}
$$

Evaluating Lemma 13 at each set of traces and applying the same arguments as given in [30] and [31, Chap. 5] mutatis mutandis leads to Theorem 13. The impact of the extra term of Lemma 12 part (4) affects only parts (2), (4), (6) and (8) - switching the $d \equiv 3$ and $d \equiv 7$ terms - but we include all of them for the sake of completeness.

Theorem 13. For $n \geq 4$ we have

$$
\text { (1) } \begin{aligned}
n I_{2}(n, 1,1,1,0) & =\sum_{\substack{d \mid n \\
d \equiv 1(8)}} \mu(d) F_{2}(n / d, 1,1,1,0)+\sum_{\substack{d \mid n \\
d \equiv 3(8)}} \mu(d) F_{2}(n / d, 1,0,0,0) \\
& +\sum_{\substack{d \mid n \\
d \equiv 5(8)}} \mu(d) F_{2}(n / d, 1,1,1,1)+\sum_{\substack{d \mid n \\
d \equiv 7(8)}} \mu(d) F_{2}(n / d, 1,0,0,1),
\end{aligned}
$$


(2) $\begin{aligned} n I_{2}(n, 1,0,0,0) & =\sum_{\substack{d \mid n \\ d \equiv 1(8)}} \mu(d) F_{2}(n / d, 1,0,0,0)+\sum_{\substack{d \mid n \\ d \equiv 3(8)}} \mu(d) F_{2}(n / d, 1,1,1,0) \\ & +\sum_{\substack{d \mid n \\ d=5(8)}} \mu(d) F_{2}(n / d, 1,0,0,1)+\sum_{\substack{d \mid n \\ d \equiv 7(8)}} \mu(d) F_{2}(n / d, 1,1,1,1),\end{aligned}$

(3) $n I_{2}(n, 1,1,1,1)=\sum_{\substack{d \mid n \\ d \equiv 1(8)}}^{d \equiv 5(8)} \mu(d) F_{2}(n / d, 1,1,1,1)+\sum_{\substack{d \mid n \\ d \equiv 3(8)}}^{d \equiv 7(8)} \mu(d) F_{2}(n / d, 1,0,0,1)$

$+\sum_{\substack{d \mid n \\ d=5(8)}} \mu(d) F_{2}(n / d, 1,1,1,0)+\sum_{\substack{d \mid n \\ d=7(8)}} \mu(d) F_{2}(n / d, 1,0,0,0)$,

(4) $n I_{2}(n, 1,0,0,1)=\sum_{\substack{d \mid n \\ d \equiv 1(8)}}^{d \equiv 5(8)} \mu(d) F_{2}(n / d, 1,0,0,1)+\sum_{\substack{d \mid n \\ d \equiv 3(8)}}^{d \equiv 7(8)} \mu(d) F_{2}(n / d, 1,1,1,1)$

$+\sum_{\substack{d \mid n \\ d=5(8)}} \mu(d) F_{2}(n / d, 1,0,0,0)+\sum_{\substack{d \mid n \\ d=7(8)}} \mu(d) F_{2}(n / d, 1,1,1,0)$,

(5) $n I_{2}(n, 1,1,0,0)=\sum_{d \mid n}^{d \equiv 5(8)} \mu(d) F_{2}(n / d, 1,1,0,0)+\sum_{d \mid n}^{d \equiv 7(8)} \mu(d) F_{2}(n / d, 1,0,1,0)$ $d \equiv 1$ (8) $\quad d \equiv 3(8)$

$+\sum_{\substack{d \mid n \\ d=5(8)}} \mu(d) F_{2}(n / d, 1,1,0,1)+\sum_{d \mid n} \mu(d) F_{2}(n / d, 1,0,1,1)$,

(6) $n I_{2}(n, 1,0,1,0)=\sum_{\substack{d \mid n \\ d \equiv 1(8)}}^{d \equiv 5(8)} \mu(d) F_{2}(n / d, 1,0,1,0)+\sum_{\substack{d \mid n \\ d \equiv 3(8)}}^{d \equiv 7(8)} \mu(d) F_{2}(n / d, 1,1,0,0)$

$+\sum_{d \mid n} \mu(d) F_{2}(n / d, 1,0,1,1)+\sum_{d \mid n} \mu(d) F_{2}(n / d, 1,1,0,1)$,

(7) $n I_{2}(n, 1,1,0,1)=\sum_{\substack{d \mid n \\ d \equiv 1(8)}}^{d \equiv 5(8)} \mu(d) F_{2}(n / d, 1,1,0,1)+\sum_{\substack{d \mid n \\ d \equiv 3(8)}}^{d \equiv 7(8)} \mu(d) F_{2}(n / d, 1,0,1,1)$

$+\sum_{d \mid n} \mu(d) F_{2}(n / d, 1,1,0,0)+\sum_{d \mid n} \mu(d) F_{2}(n / d, 1,0,1,0)$,

(8) $n I_{2}(n, 1,0,1,1)=\sum_{\substack{d \mid n \\ d \equiv 1(8)}}^{d \equiv 5(8)} \mu(d) F_{2}(n / d, 1,0,1,1)+\sum_{\substack{d \mid n \\ d \equiv 3(8)}}^{d \equiv 7(8)} \mu(d) F_{2}(n / d, 1,1,0,1)$

$+\sum_{\substack{d \mid n \\ d \equiv 5(8)}} \mu(d) F_{2}(n / d, 1,0,1,0)+\sum_{\substack{d \mid n \\ d \equiv 7(8)}} \mu(d) F_{2}(n / d, 1,1,0,0)$,

(9) $n I_{2}(n, 0,0,1,0)=\sum_{d \mid n}^{d \equiv 5(8)} \mu(d) F_{2}(n / d, 0,0,1,0)$,

(10) $n I_{2}(n, 0,0,1,1)=\sum_{d \mid n}^{d \text { odd }} \mu(d) F_{2}(n / d, 0,0,1,1)$,

(11) $n I_{2}(n, 0,1,1,1)=\sum_{d \mid n}^{d \text { odd }} \mu(d) F_{2}(n / d, 0,1,1,1)+\sum_{d \mid n} \mu(d) F_{2}(n / d, 0,1,1,0)$,

(12) $n I_{2}(n, 0,1,1,0)=\sum_{\substack{d \mid n \\ d \equiv 1(4)}}^{d \equiv 1(4)} \mu(d) F_{2}(n / d, 0,1,1,0)+\sum_{\substack{d \mid n \\ d \equiv 3(4)}}^{d \equiv 3(4)} \mu(d) F_{2}(n / d, 0,1,1,1)$, 


$$
\begin{aligned}
\text { (13) } n I_{2}(n, 0,0,0,0) & =\sum_{\substack{d|n \\
d| \text { odd }}} \mu(d) F_{2}(n / d, 0,0,0,0)-\sum_{\substack{d \mid n, \frac{n}{d} \text { even } \\
d \text { odd }}} \mu(d) F_{2}(n / 2 d, 0,0), \\
\text { (14) } n I_{2}(n, 0,0,0,1) & =\sum_{\substack{d|n \\
d| n}}^{d \text { odd }} \mu(d) F_{2}(n / d, 0,0,0,1)-\sum_{\substack{d \mid n, \frac{n}{d} \text { even } \\
\text { odd }}} \mu(d) F_{2}(n / 2 d, 0,1), \\
\text { (15) } n I_{2}(n, 0,1,0,0) & =\sum_{\substack{d \mid n \\
d \equiv 1(4)}} \mu(d) F_{2}(n / d, 0,1,0,0)-\sum_{\substack{d \mid n, \frac{n}{d} \text { even } \\
d \equiv 1(4)}} \mu(d) F_{2}(n / 2 d, 1,0) \\
& \sum_{d \mid n} \mu(d) F_{2}(n / d, 0,1,0,1)-\sum_{\substack{d \mid n, \frac{n}{d} \text { even } \\
d \equiv 3(4)}} \mu(d) F_{2}(n / 2 d, 1,1), \\
\text { (16) } n I_{2}(n, 0,1,0,1) & =\sum_{\substack{d \mid n \\
d \equiv 1(4)}} \mu(d) F_{2}(n / d, 0,1,0,1)-\sum_{\substack{d \mid n, \frac{n}{d} \text { even } \\
d \equiv 1(4)}} \mu(d) F_{2}(n / 2 d, 1,1) \\
& \sum_{\substack{d \mid n \\
d \equiv 3(4)}} \mu(d) F_{2}(n / d, 0,1,0,0)-\sum_{\substack{d \mid n, \frac{n}{d} \text { even } \\
d \equiv 3(4)}} \mu(d) F_{2}(n / 2 d, 1,0) .
\end{aligned}
$$

4.6.1 Formulae for $\boldsymbol{l} \geq \mathbf{5}$. The formulae for five coefficients follow from an argument analogous to that given in Lemma 13, using the identity

$$
T_{5}\left(f^{d}\right)=\left(\begin{array}{l}
d \\
5
\end{array}\right) T_{1}(f)+d T_{5}(f)+(d-2)\left(\begin{array}{l}
d \\
2
\end{array}\right)\left(T_{1}(f) T_{2}(f)+T_{1}(f) T_{3}(f)\right) .
$$

We omit the details since they are not part of the primary contribution of this work and follow easily. Note that Eq. (50) is at odds with Eq. (3) of [30] which claims that for any $l \geq 1$ and $d \geq 1$ the following holds $\bmod 2$ :

$$
T_{l}\left(f^{d}\right)=\sum_{k \mid l}\left(\begin{array}{l}
d \\
k
\end{array}\right) T_{l / k}(f)
$$

which contradicts Lemma 6. If combined with Lemma 6, the approach sketched in [30, §4.2] for arbitrary $l$, which uses the generalised Möbius inversion of Miers and Ruskey [40, Theorem 3.2], can no doubt be developed into a general algorithm for computing the required transforms. However, we leave describing such an algorithm, for $q=2$ and for arbitrary $q$ and $l$, as an open problem.

\section{Curves and Explicit Formulae for $q=l=3$}

In this section we determine curves and explicit formulae for all $F_{3}\left(n, t_{1}, t_{2}, t_{3}\right)$ for $n \geq 3$ but coprime to 3 and for $F_{3}(n, 0,0,0)$ for all $n \geq 3$. We do this using the indirect and direct methods, and one other method, in order to highlight some redundancy in the formulae arising from the indirect method, i.e., linear relations between powers of the roots of the featured characteristic polynomials of Frobenius. Since the Galois groups of all the featured polynomials are soluble, eliminating such redundancy is feasible, by identifying cancellations between their roots for various residues of $n \bmod 9$. On the other hand the direct method, although producing curves with harder-to-compute zeta functions, seems to have no such redundancy. This implies that there is a trade-off between the ease of computation and the compactness of the formulae.

The only previous result on such counts over $\mathbb{F}_{3}$ are due to Sharma et al. [46], who gave approximations to $F_{3}\left(n, t_{1}, *, t_{3}\right)$ using analogous simplifications to those used in [30,31]. Note that [46] contains the transforms to $I_{3}\left(n, t_{1}, *, t_{3}\right)$ and hence one can use the results of this section to compute these counts.

The reason we do not compute formulae for four coefficients is that the terms $T_{4}(\alpha)$ and $T_{4}(\beta)$ in Lemma 10 part (4) do not cancel mod 3 and the method of introducing new variables in order to linearise $T_{4}\left(a_{0}^{3}-a_{0}+r_{0}\right)$ fails as a result. 
In order to express $F_{3}\left(n, t_{1}, t_{2}, t_{3}\right)$ compactly, we define the following eight polynomials:

$$
\begin{aligned}
\epsilon_{2,1}= & X^{2}-3 X+3 \\
\epsilon_{2,2}= & X^{2}+3 X+3 \\
\epsilon_{2,3}= & X^{2}+3 \\
\epsilon_{6}= & X^{6}+3 X^{5}+9 X^{4}+15 X^{3}+27 X^{2}+27 X+27 \\
\epsilon_{12,1}= & X^{12}-3 X^{11}+3 X^{9}+9 X^{8}-45 X^{6}+81 X^{4}+81 X^{3}-729 X+729 \\
\epsilon_{12,2}= & X^{12}-3 X^{11}+12 X^{9}-18 X^{8}-27 X^{7}+117 X^{6}-81 X^{5}-162 X^{4}+324 X^{3}-729 X+729, \\
\epsilon_{12,3}= & X^{12}-3 X^{11}+9 X^{10}-15 X^{9}+36 X^{8}-54 X^{7}+117 X^{6}-162 X^{5}+324 X^{4}-405 X^{3}+729 X^{2} \\
& -729 X+729, \\
\epsilon_{12,4}= & X^{12}+6 X^{11}+18 X^{10}+39 X^{9}+63 X^{8}+81 X^{7}+117 X^{6}+243 X^{5}+567 X^{4}+1053 X^{3}+1458 X^{2} \\
& +1458 X+729 .
\end{aligned}
$$

Observe that by Theorem $5, \epsilon_{6}, \epsilon_{12,1}, \epsilon_{12,2}, \epsilon_{12,3}$ and $\epsilon_{12,4}$ are not the characteristic polynomials of the Frobenius endomorphism of supersingular abelian varieties. As in $\S 4$ we use Lemma 10 parts (1) to (3). Also as in $\S 4$, it is more efficient to compute linearised forms for $T_{2}(a)$ and $T_{3}(a)$ and to combine them as appropriate for each $i \in\left\{1, \ldots, 3^{3}-1\right\}$.

\subsection{Indirect method}

Setting $\mathbf{f}=\left(T_{3}, T_{2}, T_{1}\right)$, by the transform (12) we have

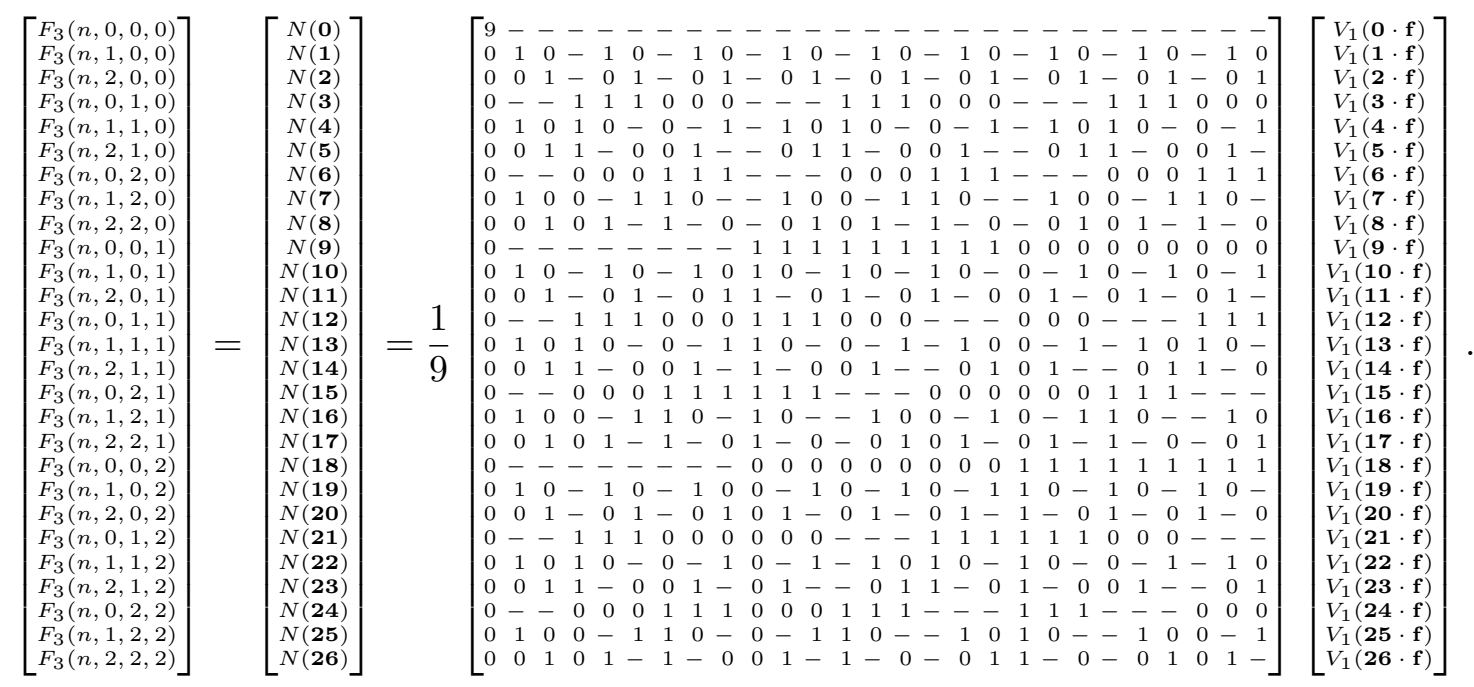

By definition we have $V_{1}(\mathbf{0} \cdot \mathbf{f})=3^{n}$, while $V_{1}(\mathbf{1} \cdot \mathbf{f})=V_{1}\left(T_{1}\right)=\#\left\{a \in \mathbb{F}_{3^{n}} \mid T_{1}(a)=1\right\}=3^{n-1}$, as does $V_{1}(\mathbf{2} \cdot \mathbf{f})=V_{1}\left(2 T_{1}\right)=\#\left\{a \in \mathbb{F}_{3^{n}} \mid 2 T_{1}(a)=1\right\}$. Note that for $r_{0} \in \mathbb{F}_{3}$ one has $T_{l}\left(r_{0}\right)=\left(\begin{array}{l}n \\ l\end{array}\right) r_{0}$. To determine $V_{1}(\mathbf{i} \cdot \mathbf{f})$ for $3 \leq i \leq 26$, setting $\alpha=a_{0}^{3}-a_{0}$ and $\beta=-r_{0}$, and using Lemma 10 parts (1) to (3) mod 3 gives the following ${ }^{\dagger}$ :

$$
\begin{aligned}
& T_{1}\left(a_{0}^{3}-a_{0}+r_{0}\right)=T_{1}\left(r_{0}\right), \\
& T_{2}\left(a_{0}^{3}-a_{0}+r_{0}\right)=T_{1}\left(a_{0}^{4}-a_{0}^{2}-r_{0}\left(\begin{array}{c}
n \\
2
\end{array}\right) / n\right), \\
& T_{3}\left(a_{0}^{3}-a_{0}+r_{0}\right)=T_{1}\left(a_{0}^{7}-a_{0}^{5}+r_{0}(n+1)\left(a_{0}^{4}-a_{0}^{2}\right)+r_{0}\left(\begin{array}{c}
n \\
3
\end{array}\right) / n\right) .
\end{aligned}
$$

\footnotetext{
${ }^{\dagger}$ See Ternary3Coefficients.mw.
} 
For $3 \leq i \leq 26$ let $\mathbf{i}=\left(i_{2}, i_{1}, i_{0}\right)$. The curves we are interested in for $a$ of trace $r_{0}$ are

$$
a_{1}^{3}-a_{1}+1 / n=i_{2}\left(a_{0}^{7}-a_{0}^{5}-r_{0}(n+1)\left(a_{0}^{4}-a_{0}^{2}\right)-r_{0}\left(\begin{array}{c}
n \\
3
\end{array}\right) / n\right)+i_{1}\left(a_{0}^{4}-a_{0}^{2}+r_{0}\left(\begin{array}{c}
n \\
2
\end{array}\right) / n\right)+i_{0} r_{0} .
$$

These curves have genus 3 if $i_{2}=0$ and genus 6 if $i_{2} \neq 0$. Corollary 1 implies that mod 3 one has

$$
\left(\left(\begin{array}{l}
n \\
3
\end{array}\right),\left(\begin{array}{l}
n \\
2
\end{array}\right),\left(\begin{array}{l}
n \\
1
\end{array}\right)\right) \equiv\left\{\begin{array}{lll}
(0,0,1) & \text { if } n \equiv 1 & (\bmod 9) \\
(0,1,-1) & \text { if } n \equiv 2 & (\bmod 9) \\
(1,0,1) & \text { if } n \equiv 4 & (\bmod 9) \\
(1,1,-1) & \text { if } n \equiv 5 & (\bmod 9) \\
(-1,0,1) & \text { if } n \equiv 7 & (\bmod 9) \\
(-1,1,-1) & \text { if } n \equiv 8 & (\bmod 9)
\end{array}\right.
$$

and hence there are six cases to consider when computing the zeta functions of each of the relevant curves. Using Magma to compute the zeta functions of the curves (55) and applying (51) gives the following theorem ${ }^{\ddagger}$, where $\mathbf{v}=\left(\rho_{n}\left(\epsilon_{2,1}\right), \rho_{n}\left(\epsilon_{2,2}\right), \rho_{n}\left(\epsilon_{2,3}\right), \rho_{n}\left(\epsilon_{6}\right), \rho_{n}\left(\epsilon_{12,1}\right), \rho_{n}\left(\epsilon_{12,2}\right), \rho_{n}\left(\epsilon_{12,3}\right), \rho_{n}\left(\epsilon_{12,4}\right)\right)$.

Theorem 14. For $n \geq 3$ we have

$$
\begin{aligned}
& F_{3}(n, 0,0,0)=3^{n-3}-\frac{1}{81}(-21,-15,-18,-8,-14,-14,-14,-8) \cdot \mathbf{v} \quad \text { if } n \equiv 1,2,4,5,7,8 \quad(\bmod 9) \\
& F_{3}(n, 1,0,0)=3^{n-3}-\frac{1}{81} \cdot\left\{\begin{array}{lrr}
(-3,3,0,4,-2,-2,-2,4) \cdot \mathbf{v} & \text { if } n \equiv 1 & (\bmod 9) \\
(3,0,-3,4,-2,4,-2,-2) \cdot \mathbf{v} & \text { if } n \equiv 2 & (\bmod 9) \\
(-3,3,0,-2,1,1,1,-2) \cdot \mathbf{v} & \text { if } n \equiv 4,7 & (\bmod 9) \\
(3,0,-3,-2,1,-2,1,1) \cdot \mathbf{v} & \text { if } n \equiv 5,8 & (\bmod 9)
\end{array}\right. \\
& F_{3}(n, 2,0,0)=3^{n-3}-\frac{1}{81} \cdot\left\{\begin{array}{lrr}
(-3,3,0,4,-2,-2,-2,4) \cdot \mathbf{v} & \text { if } n \equiv 1 & (\bmod 9) \\
(0,-3,3,4,-2,-2,4,-2) \cdot \mathbf{v} & \text { if } n \equiv 2 & (\bmod 9) \\
(-3,3,0,-2,1,1,1,-2) \cdot \mathbf{v} & \text { if } n \equiv 4,7 & (\bmod 9) \\
(0,-3,3,-2,1,1,-2,1) \cdot \mathbf{v} & \text { if } n \equiv 5,8 & (\bmod 9)
\end{array}\right. \\
& F_{3}(n, 0,1,0)=3^{n-3}-\frac{1}{81} \cdot \begin{cases}(12,9,6,4,-2,4,-2,-2) \cdot \mathbf{v} & \text { if } n \equiv 1,4,7 \quad(\bmod 9) \\
(9,6,12,-8,-14,4,10,4) \cdot \mathbf{v} & \text { if } n \equiv 2,5,8 \quad(\bmod 9)\end{cases} \\
& F_{3}(n, 1,1,0)=3^{n-3}-\frac{1}{81} \cdot\left\{\begin{array}{lrr}
(3,0,-3,-2,1,-2,1,1) \cdot \mathbf{v} & \text { if } n \equiv 1,7 \quad(\bmod 9) \\
(-3,3,0,4,-2,-2,-2,4) \cdot \mathbf{v} & \text { if } n \equiv 2 \quad(\bmod 9) \\
(3,0,-3,4,-2,4,-2,-2) \cdot \mathbf{v} & \text { if } n \equiv 4 \quad(\bmod 9) \\
(-3,3,0,-2,1,1,1,-2) \cdot \mathbf{v} & \text { if } n \equiv 5,8 \quad(\bmod 9)
\end{array}\right. \\
& F_{3}(n, 2,1,0)=3^{n-3}-\frac{1}{81} \cdot\left\{\begin{array}{lrr}
(3,0,-3,-2,1,-2,1,1) \cdot \mathbf{v} & \text { if } n \equiv 1,5,7,8 & (\bmod 9) \\
(3,0,-3,4,-2,4,-2,-2) \cdot \mathbf{v} & \text { if } n \equiv 2,4 & (\bmod 9)
\end{array}\right. \\
& F_{3}(n, 0,2,0)=3^{n-3}-\frac{1}{81} \cdot \begin{cases}(9,6,12,4,-2,-2,4,-2) \cdot \mathbf{v} & \text { if } n \equiv 1,4,7 \quad(\bmod 9) \\
(12,9,6,-8,-14,10,4,4) \cdot \mathbf{v} & \text { if } n \equiv 2,5,8 \quad(\bmod 9)\end{cases} \\
& F_{3}(n, 1,2,0)=3^{n-3}-\frac{1}{81} \cdot\left\{\begin{array}{lrr}
(0,-3,3,-2,1,1,-2,1) \cdot \mathbf{v} & \text { if } n \equiv 1,4,5,8 & (\bmod 9) \\
(0,-3,3,4,-2,-2,4,-2) \cdot \mathbf{v} & \text { if } n \equiv 2,7 & (\bmod 9)
\end{array}\right. \\
& F_{3}(n, 2,2,0)=3^{n-3}-\frac{1}{81} \cdot\left\{\begin{array}{lrr}
(0,-3,3,-2,1,1,-2,1) \cdot \mathbf{v} & \text { if } n \equiv 1,4 & (\bmod 9) \\
(-3,3,0,4,-2,-2,-2,4) \cdot \mathbf{v} & \text { if } n \equiv 2 & (\bmod 9) \\
(-3,3,0,-2,1,1,1,-2) \cdot \mathbf{v} & \text { if } n \equiv 5,8 & (\bmod 9) \\
(0,-3,3,4,-2,-2,4,-2) \cdot \mathbf{v} & \text { if } n \equiv 7 & (\bmod 9)
\end{array}\right. \\
& F_{3}(n, 0,0,1)=3^{n-3}-\frac{1}{81}(-21,-15,-18,4,7,7,7,4) \cdot \mathbf{v} \quad \text { if } n \equiv 1,2,4,5,7,8 \quad(\bmod 9)
\end{aligned}
$$

${ }^{\ddagger}$ See $\mathrm{F} 3(\mathrm{n}, \mathrm{t} 1, \mathrm{t} 2, \mathrm{t} 3)$. $\mathrm{m}$ for generation and counting code, and F3 $(\mathrm{n}, \mathrm{t} 1, \mathrm{t} 2, \mathrm{t} 3)$ verify.mw for verification of $F_{3}(n, 0,0,0)$, which can easily be adapted for the other cases. 


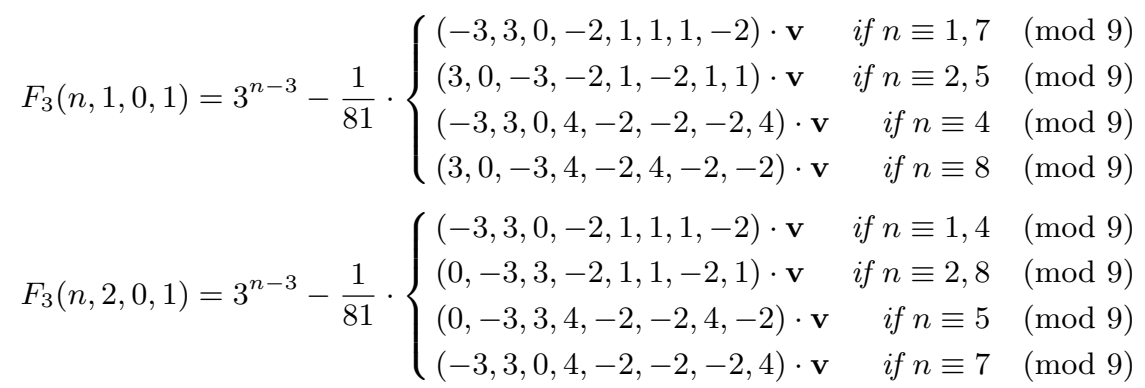

$F_{3}(n, 0,1,1)=3^{n-3}-\frac{1}{81} \cdot\left\{\begin{array}{lll}(12,9,6,-2,1,-2,1,1) \cdot \mathbf{v} & \text { if } n \equiv 1,4,7 \quad(\bmod 9) \\ (9,6,12,4,7,-2,-5,-2) \cdot \mathbf{v} & \text { if } n \equiv 2,5,8 \quad(\bmod 9)\end{array}\right.$

$F_{3}(n, 1,1,1)=3^{n-3}-\frac{1}{81} \cdot\left\{\begin{array}{lrr}(3,0,-3,-2,1,-2,1,1) \cdot \mathbf{v} & \text { if } n \equiv 1,4 & (\bmod 9) \\ (-3,3,0,-2,1,1,1,-2) \cdot \mathbf{v} & \text { if } n \equiv 2,5 & (\bmod 9) \\ (3,0,-3,4,-2,4,-2,-2) \cdot \mathbf{v} & \text { if } n \equiv 7 & (\bmod 9) \\ (-3,3,0,4,-2,-2,-2,4) \cdot \mathbf{v} & \text { if } n \equiv 8 & (\bmod 9)\end{array}\right.$

$F_{3}(n, 2,1,1)=3^{n-3}-\frac{1}{81} \cdot\left\{\begin{array}{lrr}(3,0,-3,4,-2,4,-2,-2) \cdot \mathbf{v} & \text { if } n \equiv 1,5 \quad(\bmod 9) \\ (3,0,-3,-2,1,-2,1,1) \cdot \mathbf{v} & \text { if } n \equiv 2,4,7,8 \quad(\bmod 9)\end{array}\right.$

$F_{3}(n, 0,2,1)=3^{n-3}-\frac{1}{81} \cdot\left\{\begin{array}{lll}(9,6,12,-2,1,1,-2,1) \cdot \mathbf{v} & \text { if } n \equiv 1,4,7 \quad(\bmod 9) \\ (12,9,6,4,7,-5,-2,-2) \cdot \mathbf{v} & \text { if } n \equiv 2,5,8 \quad(\bmod 9)\end{array}\right.$

$F_{3}(n, 1,2,1)=3^{n-3}-\frac{1}{81} \cdot\left\{\begin{array}{lrr}(0,-3,3,4,-2,-2,4,-2) \cdot \mathbf{v} & \text { if } n \equiv 1,8 \quad(\bmod 9) \\ (0,-3,3,-2,1,1,-2,1) \cdot \mathbf{v} & \text { if } n \equiv 2,4,5,7 \quad(\bmod 9)\end{array}\right.$

$F_{3}(n, 2,2,1)=3^{n-3}-\frac{1}{81} \cdot\left\{\begin{array}{lrr}(0,-3,3,-2,1,1,-2,1) \cdot \mathbf{v} & \text { if } n \equiv 1,7 & (\bmod 9) \\ (-3,3,0,-2,1,1,1,-2) \cdot \mathbf{v} & \text { if } n \equiv 2,8 & (\bmod 9) \\ (0,-3,3,4,-2,-2,4,-2) \cdot \mathbf{v} & \text { if } n \equiv 4 & (\bmod 9) \\ (-3,3,0,4,-2,-2,-2,4) \cdot \mathbf{v} & \text { if } n \equiv 5 & (\bmod 9)\end{array}\right.$

$F_{3}(n, 0,0,2)=3^{n-3}-\frac{1}{81}(-21,-15,-18,4,7,7,7,4) \cdot \mathbf{v} \quad$ if $n \equiv 1,2,4,5,7,8 \quad(\bmod 9)$

$F_{3}(n, 1,0,2)=3^{n-3}-\frac{1}{81} \cdot\left\{\begin{array}{lrr}(-3,3,0,-2,1,1,1,-2) \cdot \mathbf{v} & \text { if } n \equiv 1,4 & (\bmod 9) \\ (3,0,-3,-2,1,-2,1,1) \cdot \mathbf{v} & \text { if } n \equiv 2,8 & (\bmod 9) \\ (3,0,-3,4,-2,4,-2,-2) \cdot \mathbf{v} & \text { if } n \equiv 5 & (\bmod 9) \\ (-3,3,0,4,-2,-2,-2,4) \cdot \mathbf{v} & \text { if } n \equiv 7 & (\bmod 9)\end{array}\right.$

$F_{3}(n, 2,0,2)=3^{n-3}-\frac{1}{81} \cdot\left\{\begin{array}{lrr}(-3,3,0,-2,1,1,1,-2) \cdot \mathbf{v} & \text { if } n \equiv 1,7 & (\bmod 9) \\ (0,-3,3,-2,1,1,-2,1) \cdot \mathbf{v} & \text { if } n \equiv 2,5 & (\bmod 9) \\ (-3,3,0,4,-2,-2,-2,4) \cdot \mathbf{v} & \text { if } n \equiv 4 & (\bmod 9) \\ (0,-3,3,4,-2,-2,4,-2) \cdot \mathbf{v} & \text { if } n \equiv 8 & (\bmod 9)\end{array}\right.$

$F_{3}(n, 0,1,2)=3^{n-3}-\frac{1}{81} \cdot\left\{\begin{array}{lll}(12,9,6,-2,1,-2,1,1) \cdot \mathbf{v} & \text { if } n \equiv 1,4,7 \quad(\bmod 9) \\ (9,6,12,4,7,-2,-5,-2) \cdot \mathbf{v} & \text { if } n \equiv 2,5,8 \quad(\bmod 9)\end{array}\right.$

$F_{3}(n, 1,1,2)=3^{n-3}-\frac{1}{81} \cdot\left\{\begin{array}{lrr}(3,0,-3,4,-2,4,-2,-2) \cdot \mathbf{v} & \text { if } n \equiv 1 & (\bmod 9) \\ (-3,3,0,-2,1,1,1,-2) \cdot \mathbf{v} & \text { if } n \equiv 2,8 & (\bmod 9) \\ (3,0,-3,-2,1,-2,1,1) \cdot \mathbf{v} & \text { if } n \equiv 4,7 & (\bmod 9) \\ (-3,3,0,4,-2,-2,-2,4) \cdot \mathbf{v} & \text { if } n \equiv 5 & (\bmod 9)\end{array}\right.$

$F_{3}(n, 2,1,2)=3^{n-3}-\frac{1}{81} \cdot\left\{\begin{array}{lrr}(3,0,-3,-2,1,-2,1,1) \cdot \mathbf{v} & \text { if } n \equiv 1,2,4,5 & (\bmod 9) \\ (3,0,-3,4,-2,4,-2,-2) \cdot \mathbf{v} & \text { if } n \equiv 7,8 & (\bmod 9)\end{array}\right.$

$F_{3}(n, 0,2,2)=3^{n-3}-\frac{1}{81} \cdot\left\{\begin{array}{lll}(9,6,12,-2,1,1,-2,1) \cdot \mathbf{v} & \text { if } n \equiv 1,4,7 \quad(\bmod 9) \\ (12,9,6,4,7,-5,-2,-2) \cdot \mathbf{v} & \text { if } n \equiv 2,5,8 \quad(\bmod 9)\end{array}\right.$ 


$$
\begin{gathered}
F_{3}(n, 1,2,2)=3^{n-3}-\frac{1}{81} \cdot\left\{\begin{array}{lc}
(0,-3,3,-2,1,1,-2,1) \cdot \mathbf{v} & \text { if } n \equiv 1,2,7,8 \quad(\bmod 9) \\
(0,-3,3,4,-2,-2,4,-2) \cdot \mathbf{v} & \text { if } n \equiv 4,5 \quad(\bmod 9)
\end{array}\right. \\
F_{3}(n, 2,2,2)=3^{n-3}-\frac{1}{81} \cdot\left\{\begin{array}{lcc}
(0,-3,3,4,-2,-2,4,-2) \cdot \mathbf{v} & \text { if } n \equiv 1 \quad(\bmod 9) \\
(-3,3,0,-2,1,1,1,-2) \cdot \mathbf{v} & \text { if } n \equiv 2,5 \quad(\bmod 9) \\
(0,-3,3,-2,1,1,-2,1) \cdot \mathbf{v} & \text { if } n \equiv 4,7 \quad(\bmod 9) \\
(-3,3,0,4,-2,-2,-2,4) \cdot \mathbf{v} & \text { if } n \equiv 8 \quad(\bmod 9)
\end{array}\right.
\end{gathered}
$$

5.1.1 Less redundant formulae. An alternative and more representationally efficient way to evaluate these formulae is to write:

$$
\begin{aligned}
F_{3}\left(n, t_{1}, t_{2}, t_{3}\right)= & \frac{1}{3} \#\left\{a_{0} \in \mathbb{F}_{3^{n}} \mid T_{1}\left(a_{0}^{4}-a_{0}^{2}-t_{1}\left(\begin{array}{c}
n \\
2
\end{array}\right) / n^{2}\right)=t_{2},\right. \\
& \left.T_{1}\left(a_{0}^{7}-a_{0}^{5}+t_{1}(n+1)\left(a_{0}^{4}-a_{0}^{2}\right) / n+t_{1}\left(\begin{array}{c}
n \\
3
\end{array}\right) / n^{2}\right)=t_{3}\right\},
\end{aligned}
$$

and then for each $t_{1}$ apply the indirect method to compute all nine $F_{3}\left(n, t_{1}, t_{2}, t_{3}\right)$ simultaneously. In particular, we instead let $\mathbf{f}=\left(a_{0}^{7}-a_{0}^{5}+t_{1}(n+1)\left(a_{0}^{4}-a_{0}^{2}\right) / n+t_{1}\left(\begin{array}{c}n \\ 3\end{array}\right) / n^{2}, a_{0}^{4}-a_{0}^{2}-t_{1}\left(\begin{array}{c}n \\ 2\end{array}\right) / n^{2}\right)$. Since there are now fewer summands, the total degree of the expressions is 84 in the worst case: for $n \geq 3$ and coprime to 3 we have

$$
F_{3}(n, 0,0,0)=3^{n-3}+\frac{1}{27}\left(3 \rho_{n}\left(\epsilon_{2,1}\right)+\rho_{n}\left(\epsilon_{2,2}\right)+2 \rho_{n}\left(\epsilon_{2,3}\right)+2 \rho_{n}\left(\epsilon_{12,1}\right)+2 \rho_{n}\left(\epsilon_{12,2}\right)+2 \rho_{n}\left(\epsilon_{12,3}\right)\right) .
$$

Comparing this with Theorem 14 implies the identity, valid for all $n \equiv 1,2,4,5,7,8(\bmod 9)$ :

$$
3\left(\rho_{n}\left(\epsilon_{2,1}\right)+\rho_{n}\left(\epsilon_{2,2}\right)+\rho_{n}\left(\epsilon_{2,3}\right)\right)+2\left(\rho_{n}\left(\epsilon_{6}\right)+\rho_{n}\left(\epsilon_{12,1}\right)+\rho_{n}\left(\epsilon_{12,2}\right)+\rho_{n}\left(\epsilon_{12,3}\right)+\rho_{n}\left(\epsilon_{12,4}\right)\right)=0 .
$$

This is therefore an example of a linear relation between the powers of the roots of the featured characteristic polynomials of Frobenius. Furthermore, since the roots of $\epsilon_{2,1}, \epsilon_{2,2}, \epsilon_{2,3}$ are 12-th roots of unity, one can check that

$$
\rho_{n}\left(\epsilon_{2,1}\right)+\rho_{n}\left(\epsilon_{2,2}\right)+\rho_{n}\left(\epsilon_{2,3}\right)=\left\{\begin{array}{l}
6 \cdot 3^{n / 2} \text { if } n \equiv 0 \quad(\bmod 12) \\
-6 \cdot 3^{n / 2} \text { if } n \equiv 6 \quad(\bmod 12) \\
0 \text { otherwise }
\end{array}\right.
$$

We therefore deduce that for all $n \equiv 1,2,4,5,7,8(\bmod 9)$, we have

$$
\rho_{n}\left(\epsilon_{6}\right)+\rho_{n}\left(\epsilon_{12,1}\right)+\rho_{n}\left(\epsilon_{12,2}\right)+\rho_{n}\left(\epsilon_{12,3}\right)+\rho_{n}\left(\epsilon_{12,4}\right)=0,
$$

which does not seem obvious from the polynomials themselves.

\subsection{Direct method}

For $n \geq 3$ and coprime to 3 applying Equations (52) to (54) we have

$$
\begin{aligned}
F_{3}\left(n, t_{1}, t_{2}, t_{3}\right)= & \#\left\{a \in \mathbb{F}_{3^{n}} \mid T_{1}(a)=t_{1}, T_{2}(a)=t_{2}, T_{3}(a)=t_{3}\right\} \\
= & \frac{1}{3} \#\left\{a_{0} \in \mathbb{F}_{3^{n}} \mid T_{2}\left(a_{0}^{3}-a_{0}+t_{1} / \bar{n}\right)=t_{2}, T_{3}\left(a_{0}^{3}-a_{0}+t_{1} / n\right)=t_{3}\right\} \\
= & \frac{1}{3^{3}} \#\left\{\left(a_{0}, a_{1}, a_{2}\right) \in\left(\mathbb{F}_{3^{n}}\right)^{3} \mid a_{1}^{3}-a_{1}+t_{2} / n=a_{0}^{4}-a_{0}^{2}-t_{1}\left(\begin{array}{c}
n \\
2
\end{array}\right) / n^{2},\right. \\
& \left.a_{2}^{3}-a_{2}+t_{3} / n=a_{0}^{7}-a_{0}^{5}+t_{1}(n+1)\left(a_{0}^{4}-a_{0}^{2}\right) / n+t_{1}\left(\begin{array}{c}
n \\
3
\end{array}\right) / n^{2}\right\} .
\end{aligned}
$$


These curves are all absolutely irreducible and of genus 21 , which is much less than half of the total degree of each expression in Theorem 14 and precisely half the degree of the worst case of the alternative indirect method of §5.1.1. Based on some preliminary experiments, Magma can compute the zeta functions of the curves (57) in a matter of days. However, in order to compute these functions more efficiently, since there are only two linear conditions one can apply [3, Lemma 6]. In particular, we have

$$
F_{3}\left(n, t_{1}, t_{2}, t_{3}\right)=\frac{1}{9}\left(V((0,1) \cdot \mathbf{f})+\sum_{\alpha \in \mathbb{F}_{3}} V((1, \alpha) \cdot \mathbf{f})-3^{n}\right),
$$

where $\mathbf{f}=\left(a_{0}^{7}-a_{0}^{5}+t_{1}(n+1)\left(a_{0}^{4}-a_{0}^{2}\right) / n+t_{1}\left(\begin{array}{c}n \\ 3\end{array}\right) / n^{2}, a_{0}^{4}-a_{0}^{2}-t_{1}\left(\begin{array}{c}n \\ 2\end{array}\right) / n^{2}\right)$. Since this uses the zero count $V(\mathbf{i} \cdot \mathbf{f})$ the resulting formula for $F_{3}(n, 0,0,0)$ is valid for all $n \geq 3$. This leads to the following refinement of Theorem 14.

Theorem 15. For $n \geq 3$ we have

$$
\begin{aligned}
& F_{3}(n, 0,0,0)=3^{n-3}-\frac{1}{27}(0,2,1,2,0,0,0,2) \cdot \mathbf{v} \text { for all } n \geq 3 \\
& F_{3}(n, 1,0,0)=3^{n-3}-\frac{1}{27} \cdot\left\{\begin{array}{rrr}
(0,2,1,2,0,0,0,2) \cdot \mathbf{v} & \text { if } n \equiv 1 \quad(\bmod 9) \\
(2,1,0,2,0,2,0,0) \cdot \mathbf{v} & \text { if } n \equiv 2 \quad(\bmod 9) \\
(0,2,1,0,1,1,1,0) \cdot \mathbf{v} & \text { if } n \equiv 4,7 \quad(\bmod 9) \\
(2,1,0,0,1,0,1,1) \cdot \mathbf{v} & \text { if } n \equiv 5,8 \quad(\bmod 9)
\end{array}\right. \\
& F_{3}(n, 2,0,0)=3^{n-3}-\frac{1}{27} \cdot\left\{\begin{array}{rrr}
(0,2,1,2,0,0,0,2) \cdot \mathbf{v} & \text { if } n \equiv 1 \quad(\bmod 9) \\
(1,0,2,2,0,0,2,0) \cdot \mathbf{v} & \text { if } n \equiv 2 \quad(\bmod 9) \\
(0,2,1,0,1,1,1,0) \cdot \mathbf{v} & \text { if } n \equiv 4,7 \quad(\bmod 9) \\
(1,0,2,0,1,1,0,1) \cdot \mathbf{v} & \text { if } n \equiv 5,8 & (\bmod 9)
\end{array}\right. \\
& F_{3}(n, 0,1,0)=3^{n-3}-\frac{1}{27} \cdot \begin{cases}(2,1,0,2,0,2,0,0) \cdot \mathbf{v} & \text { if } n \equiv 1,4,7 \quad(\bmod 9) \\
(1,0,2,2,0,0,2,0) \cdot \mathbf{v} & \text { if } n \equiv 2,5,8 \quad(\bmod 9)\end{cases} \\
& F_{3}(n, 1,1,0)=3^{n-3}-\frac{1}{27} \cdot\left\{\begin{array}{lrr}
(2,1,0,0,1,0,1,1) \cdot \mathbf{v} & \text { if } n \equiv 1,7 \quad(\bmod 9) \\
(0,2,1,2,0,0,0,2) \cdot \mathbf{v} & \text { if } n \equiv 2 \quad(\bmod 9) \\
(2,1,0,2,0,2,0,0) \cdot \mathbf{v} & \text { if } n \equiv 4 \quad(\bmod 9) \\
(0,2,1,0,1,1,1,0) \cdot \mathbf{v} & \text { if } n \equiv 5,8 \quad(\bmod 9)
\end{array}\right. \\
& F_{3}(n, 2,1,0)=3^{n-3}-\frac{1}{27} \cdot\left\{\begin{array}{rr}
(2,1,0,0,1,0,1,1) \cdot \mathbf{v} & \text { if } n \equiv 1,5,7,8 \quad(\bmod 9) \\
(2,1,0,2,0,2,0,0) \cdot \mathbf{v} & \text { if } n \equiv 2,4 \quad(\bmod 9)
\end{array}\right. \\
& F_{3}(n, 0,2,0)=3^{n-3}-\frac{1}{27} \cdot \begin{cases}(1,0,2,2,0,0,2,0) \cdot \mathbf{v} & \text { if } n \equiv 1,4,7 \quad(\bmod 9) \\
(2,1,0,2,0,2,0,0) \cdot \mathbf{v} & \text { if } n \equiv 2,5,8 \quad(\bmod 9)\end{cases} \\
& F_{3}(n, 1,2,0)=3^{n-3}-\frac{1}{27} \cdot\left\{\begin{array}{rrr}
(1,0,2,0,1,1,0,1) \cdot \mathbf{v} & \text { if } n \equiv 1,4,5,8 \quad(\bmod 9) \\
(1,0,2,2,0,0,2,0) \cdot \mathbf{v} & \text { if } n \equiv 2,7 & (\bmod 9)
\end{array}\right. \\
& F_{3}(n, 2,2,0)=3^{n-3}-\frac{1}{27} \cdot\left\{\begin{array}{lrr}
(1,0,2,0,1,1,0,1) \cdot \mathbf{v} & \text { if } n \equiv 1,4 \quad(\bmod 9) \\
(0,2,1,2,0,0,0,2) \cdot \mathbf{v} & \text { if } n \equiv 2 \quad(\bmod 9) \\
(0,2,1,0,1,1,1,0) \cdot \mathbf{v} & \text { if } n \equiv 5,8 \quad(\bmod 9) \\
(1,0,2,2,0,0,2,0) \cdot \mathbf{v} & \text { if } n \equiv 7 \quad(\bmod 9)
\end{array}\right. \\
& F_{3}(n, 0,0,1)=3^{n-3}-\frac{1}{27}(0,2,1,0,1,1,1,0) \cdot \mathbf{v} \quad \text { if } n \equiv 1,2,4,5,7,8 \quad(\bmod 9) \\
& F_{3}(n, 1,0,1)=3^{n-3}-\frac{1}{27} \cdot\left\{\begin{array}{rrr}
(0,2,1,0,1,1,1,0) \cdot \mathbf{v} & \text { if } n \equiv 1,7 \quad(\bmod 9) \\
(2,1,0,0,1,0,1,1) \cdot \mathbf{v} & \text { if } n \equiv 2,5 \quad(\bmod 9) \\
(0,2,1,2,0,0,0,2) \cdot \mathbf{v} & \text { if } n \equiv 4 \quad(\bmod 9) \\
(2,1,0,2,0,2,0,0) \cdot \mathbf{v} & \text { if } n \equiv 8 \quad(\bmod 9)
\end{array}\right. \\
& F_{3}(n, 2,0,1)=3^{n-3}-\frac{1}{27} \cdot\left\{\begin{array}{rrr}
(0,2,1,0,1,1,1,0) \cdot \mathbf{v} & \text { if } n \equiv 1,4 \quad(\bmod 9) \\
(1,0,2,0,1,1,0,1) \cdot \mathbf{v} & \text { if } n \equiv 2,8 \quad(\bmod 9) \\
(1,0,2,2,0,0,2,0) \cdot \mathbf{v} & \text { if } n \equiv 5 \quad(\bmod 9) \\
(0,2,1,2,0,0,0,2) \cdot \mathbf{v} & \text { if } n \equiv 7 \quad(\bmod 9)
\end{array}\right.
\end{aligned}
$$




$$
\begin{aligned}
& F_{3}(n, 0,1,1)=3^{n-3}-\frac{1}{27} \cdot\left\{\begin{array}{l}
(2,1,0,0,1,0,1,1) \cdot \mathbf{v} \quad \text { if } n \equiv 1,4,7 \quad(\bmod 9) \\
(1,0,2,0,1,1,0,1) \cdot \mathbf{v} \quad \text { if } n \equiv 2,5,8 \quad(\bmod 9)
\end{array}\right. \\
& F_{3}(n, 1,1,1)=3^{n-3}-\frac{1}{27} \cdot\left\{\begin{array}{rrr}
(2,1,0,0,1,0,1,1) \cdot \mathbf{v} & \text { if } n \equiv 1,4 & (\bmod 9) \\
(0,2,1,0,1,1,1,0) \cdot \mathbf{v} & \text { if } n \equiv 2,5 & (\bmod 9) \\
(2,1,0,2,0,2,0,0) \cdot \mathbf{v} & \text { if } n \equiv 7 & (\bmod 9) \\
(0,2,1,2,0,0,0,2) \cdot \mathbf{v} & \text { if } n \equiv 8 & (\bmod 9)
\end{array}\right. \\
& F_{3}(n, 2,1,1)=3^{n-3}-\frac{1}{27} \cdot\left\{\begin{array}{rrr}
(2,1,0,2,0,2,0,0) \cdot \mathbf{v} & \text { if } n \equiv 1,5 \quad(\bmod 9) \\
(2,1,0,0,1,0,1,1) \cdot \mathbf{v} & \text { if } n \equiv 2,4,7,8 \quad(\bmod 9)
\end{array}\right. \\
& F_{3}(n, 0,2,1)=3^{n-3}-\frac{1}{27} \cdot \begin{cases}(1,0,2,0,1,1,0,1) \cdot \mathbf{v} & \text { if } n \equiv 1,4,7 \quad(\bmod 9) \\
(2,1,0,0,1,0,1,1) \cdot \mathbf{v} & \text { if } n \equiv 2,5,8 \quad(\bmod 9)\end{cases} \\
& F_{3}(n, 1,2,1)=3^{n-3}-\frac{1}{27} \cdot\left\{\begin{array}{rrr}
(1,0,2,2,0,0,2,0) \cdot \mathbf{v} & \text { if } n \equiv 1,8 \quad(\bmod 9) \\
(1,0,2,0,1,1,0,1) \cdot \mathbf{v} & \text { if } n \equiv 2,4,5,7 & (\bmod 9)
\end{array}\right. \\
& F_{3}(n, 2,2,1)=3^{n-3}-\frac{1}{27} \cdot\left\{\begin{array}{rrr}
(1,0,2,0,1,1,0,1) \cdot \mathbf{v} & \text { if } n \equiv 1,7 & (\bmod 9) \\
(0,2,1,0,1,1,1,0) \cdot \mathbf{v} & \text { if } n \equiv 2,8 & (\bmod 9) \\
(1,0,2,2,0,0,2,0) \cdot \mathbf{v} & \text { if } n \equiv 4 & (\bmod 9) \\
(0,2,1,2,0,0,0,2) \cdot \mathbf{v} & \text { if } n \equiv 5 & (\bmod 9)
\end{array}\right. \\
& F_{3}(n, 0,0,2)=3^{n-3}-\frac{1}{27}(0,2,1,0,1,1,1,0) \cdot \mathbf{v} \quad \text { if } n \equiv 1,2,4,5,7,8 \quad(\bmod 9) \\
& F_{3}(n, 1,0,2)=3^{n-3}-\frac{1}{27} \cdot\left\{\begin{array}{rrr}
(0,2,1,0,1,1,1,0) \cdot \mathbf{v} & \text { if } n \equiv 1,4 \quad(\bmod 9) \\
(2,1,0,0,1,0,1,1) \cdot \mathbf{v} & \text { if } n \equiv 2,8 \quad(\bmod 9) \\
(2,1,0,2,0,2,0,0) \cdot \mathbf{v} & \text { if } n \equiv 5 \quad(\bmod 9) \\
(0,2,1,2,0,0,0,2) \cdot \mathbf{v} & \text { if } n \equiv 7 \quad(\bmod 9)
\end{array}\right. \\
& F_{3}(n, 2,0,2)=3^{n-3}-\frac{1}{27} \cdot\left\{\begin{array}{rrr}
(0,2,1,0,1,1,1,0) \cdot \mathbf{v} & \text { if } n \equiv 1,7 & (\bmod 9) \\
(1,0,2,0,1,1,0,1) \cdot \mathbf{v} & \text { if } n \equiv 2,5 & (\bmod 9) \\
(0,2,1,2,0,0,0,2) \cdot \mathbf{v} & \text { if } n \equiv 4 & (\bmod 9) \\
(1,0,2,2,0,0,2,0) \cdot \mathbf{v} & \text { if } n \equiv 8 & (\bmod 9)
\end{array}\right. \\
& F_{3}(n, 0,1,2)=3^{n-3}-\frac{1}{27} \cdot\left\{\begin{array}{lll}
(2,1,0,0,1,0,1,1) \cdot \mathbf{v} & \text { if } n \equiv 1,4,7 \quad(\bmod 9) \\
(1,0,2,0,1,1,0,1) \cdot \mathbf{v} & \text { if } n \equiv 2,5,8 \quad(\bmod 9)
\end{array}\right. \\
& F_{3}(n, 1,1,2)=3^{n-3}-\frac{1}{27} \cdot\left\{\begin{array}{rrr}
(2,1,0,2,0,2,0,0) \cdot \mathbf{v} & \text { if } n \equiv 1 & (\bmod 9) \\
(0,2,1,0,1,1,1,0) \cdot \mathbf{v} & \text { if } n \equiv 2,8 & (\bmod 9) \\
(2,1,0,0,1,0,1,1) \cdot \mathbf{v} & \text { if } n \equiv 4,7 & (\bmod 9) \\
([0,2,1,2,0,0,0,2) \cdot \mathbf{v} & \text { if } n \equiv 5 & (\bmod 9)
\end{array}\right. \\
& F_{3}(n, 2,1,2)=3^{n-3}-\frac{1}{27} \cdot\left\{\begin{array}{rrr}
(2,1,0,0,1,0,1,1) \cdot \mathbf{v} & \text { if } n \equiv 1,2,4,5 & (\bmod 9) \\
(2,1,0,2,0,2,0,0) \cdot \mathbf{v} & \text { if } n \equiv 7,8 & (\bmod 9)
\end{array}\right. \\
& F_{3}(n, 0,2,2)=3^{n-3}-\frac{1}{27} \cdot\left\{\begin{array}{lll}
(1,0,2,0,1,1,0,1) \cdot \mathbf{v} & \text { if } n \equiv 1,4,7 \quad(\bmod 9) \\
(2,1,0,0,1,0,1,1) \cdot \mathbf{v} & \text { if } n \equiv 2,5,8 \quad(\bmod 9)
\end{array}\right. \\
& F_{3}(n, 1,2,2)=3^{n-3}-\frac{1}{27} \cdot\left\{\begin{array}{rrr}
(1,0,2,0,1,1,0,1) \cdot \mathbf{v} & \text { if } n \equiv 1,2,7,8 \quad(\bmod 9) \\
(1,0,2,2,0,0,2,0) \cdot \mathbf{v} & \text { if } n \equiv 4,5 \quad(\bmod 9)
\end{array}\right. \\
& F_{3}(n, 2,2,2)=3^{n-3}-\frac{1}{27} \cdot\left\{\begin{array}{rrr}
(1,0,2,2,0,0,2,0) \cdot \mathbf{v} & \text { if } n \equiv 1 & (\bmod 9) \\
(0,2,1,0,1,1,1,0) \cdot \mathbf{v} & \text { if } n \equiv 2,5 & (\bmod 9) \\
(1,0,2,0,1,1,0,1) \cdot \mathbf{v} & \text { if } n \equiv 4,7 & (\bmod 9) \\
(0,2,1,2,0,0,0,2) \cdot \mathbf{v} & \text { if } n \equiv 8 & (\bmod 9)
\end{array}\right.
\end{aligned}
$$

Observe that the total degrees of the featured polynomials in Theorem 15 is always 42 , which means these are the characteristic polynomials of Frobenius arising from the direct method and should have optimal representational efficiency. 
If one compares for instance the formulae for $F_{3}(n, 0,1,2)$ in Theorems 14 and 15 one can further deduce that for all $n \equiv 2,5,8(\bmod 9)$, we have $\rho_{n}\left(\epsilon_{6}\right)+\rho_{n}\left(\epsilon_{12,1}\right)=0$ and $\rho_{n}\left(\epsilon_{12,2}\right)+\rho_{n}\left(\epsilon_{12,3}\right)+\rho_{n}\left(\epsilon_{12,4}\right)=0$, which again are seemingly not obvious from the polynomials alone. The three observed identities and possibly any others arising by such a comparison would probably allow one to transform Theorem 14 into Theorem 15 without carrying out any zeta function computations. Conversely, although our goal was to compute formulae for $F_{3}\left(n, t_{1}, t_{2}, t_{3}\right)$, by using various approaches to do so one can also deduce identities between the roots of the featured characteristic polynomials of Frobenius for residues of $n$ mod 9 without computing any of the roots explicitly.

\section{Final Remarks and Open Problems}

We have presented the first algorithmic approaches to solving the prescribed traces problem and therefore the prescribed coefficients problem. While our main algorithm for $l<p$ is extremely simple to state and very efficient, the $l \geq p$ case in full generality remains open. There are many properties and consequences of our approaches that have yet to be determined or explored. We now present some open problems, in what may be considered to be an approximately increasing order of interest.

Problem 1: Provide an algorithm which for any $q=p^{r}$ and $l$ outputs the transforms between $I_{q}\left(n, t_{1}, \ldots, t_{l}\right)$ and $F_{q}\left(n, t_{1}, \ldots, t_{l}\right)$, and deduce its complexity.

Problem 2: Compute the characteristic polynomials of Frobenius of the curves arising for $F_{2}\left(n, t_{1}, \ldots, t_{6}\right)$ and $F_{2}\left(n, t_{1}, \ldots, t_{7}\right)$, for $n$ odd. Although it is feasible to compute these by brute force point counting, it would be preferable to employ a more elegant approach, perhaps by computing the quotient curves arising from various curve automorphisms and then applying a theorem of Kani and Rosen [28] to infer the decomposition of their Jacobians.

Problem 3: Compute formulae for $F_{2}\left(n, t_{1}, \ldots, t_{l}\right)$ with $4 \leq l \leq 7$ for all $n \geq l$.

Problem 4: More generally, for the main algorithm provide a method for solving the $n \equiv 0(\bmod p)$ cases, for any $q \geq 2$. Note that if solved then the formulae for $F_{q}\left(n, t_{1}, \ldots, t_{l}\right)$ for each of the $p$ residue classes of $n$ can be unified via Fourier analysis using the complex $p$-th roots of unity, as in [3]. Similarly, if $l \geq p$ then as per Corollary 1 the complex $p^{1+\left\lfloor\log _{p} l\right\rfloor}$ th roots of unity can be employed. However, it may be preferable to retain the residue class distinctions for simplicity (cf. [3, Prop. 4 and 5]).

Problem 5: Determine if it is possible to express $T_{l}(\alpha-\beta)$ in terms of lower degree traces, over $\mathbb{Z}$, for any or all $l>7$, so that one may extend the $q=2$ approach.

Problem 6: For the indirect method in the main algorithm when $q>p$, remove the reliance on Lemma 5 so that the $\frac{q-1}{p-1}$ factor in the sum in (1) in Theorem 1 may be removed.

Problem 7: When $l<p$ provide an algorithm to compute the zeta function of the curve (7) arising from the direct method, or indeed of the fibre product (6), which is more efficient for a single $F_{q}\left(n, t_{1}, \ldots, t_{l}\right)$ than executing the indirect method. This is also desirable since the direct method seems to produce the most compact formulae.

Problem 8: As is evident from the examples we have given for $q=2$, there are often several ways to associate an affine curve $C_{\mathbf{r}, \bar{n}}$ to a given counting problem $F_{q}\left(n, t_{l_{0}}, \ldots, t_{l_{m-1}}\right)$ with differing numbers of auxiliary variables $\left(a_{0}, \ldots, a_{s-1}\right)$ with corresponding linear traces $\mathbf{r}=\left(r_{0}, \ldots, r_{s-1}\right)$. Not only does this affect the complexity of determining the relevant characteristic values, but each such curve may lead to different (but necessarily numerically identical) formulae. One can associate a generating, or zeta function to each prescribed traces problem, by letting $N_{n}=q^{m} \cdot F_{q}\left(n, t_{l_{0}}, \ldots, t_{l_{m-1}}\right)+1$ and as usual defining

$$
Z\left(F_{q}\left(n, t_{l_{0}}, \ldots, t_{l_{m-1}}\right) ; t\right)=\exp \left(\sum_{n=1}^{\infty} \frac{N_{n}}{n} t^{n}\right) \text {. }
$$


If $C_{\mathbf{r}, \bar{n}}$ is absolutely irreducible for each $\mathbf{r}$ and assuming Problem 4 has been solved then for all $\bar{n} \in$ $\{0, \ldots, p-1\}$ one has:

$$
Z\left(F_{q}\left(n, t_{l_{0}}, \ldots, t_{l_{m-1}}\right) ; t\right)=\frac{L_{\bar{n}}(t)^{1 / q^{s}}}{(1-t)(1-q t)}, \text { for all } n \equiv \bar{n} \quad(\bmod p) \text { with } n \geq l_{m-1},
$$

where $L_{\bar{n}}(t)=c_{0}+c_{1} t+\cdots+c_{2 g} t^{2 g} \in \mathbb{Z}[t]$ with $c_{0}=1$ is the product of the characteristic polynomials of Frobenius of the $q^{s}$ specialisations of $C_{\mathbf{r}, \bar{n}}$. Since $L_{\bar{n}}(t)$ and $s$ in Eq. (58) are non-canonical, this is less than aesthetically pleasing in comparison to the rational zeta functions of smooth projective curves. Is there a canonical way to associate such a curve, which also avoids all redundancy? Perhaps one can associate a motivic zeta function to each $F_{q}\left(n, t_{l_{0}}, \ldots, t_{l_{m-1}}\right)$ à la $[14,15]$, which resolve a similar non-canonicality issue when associating varieties to counting problems in the theory of $p$-groups and nilpotent groups?

Problem 9: Regarding the general $l \geq p$ case, another natural question is whether or not it is possible to obviate the failure of Newton's identities by working $p$-adically and in Galois rings, à la Fan and Han's refinement [16] of Han's work on Cohen's problem [55]? See also the exposition of Cohen [11]. If so, this could lead to an algorithm for solving the prescribed traces problem for any number of coefficients in any positions. Assuming this can be done, for the sake of generality we make the following conjecture.

Conjecture 1. For every $q=p^{r}, 1 \leq l_{0}<\cdots<l_{m-1},\left(t_{l_{0}}, \ldots, t_{l_{m-1}}\right) \in\left(\mathbb{F}_{q}\right)^{m}$ and $\bar{n} \in\left\{0, \ldots, p^{1+\left\lfloor\log _{p} l_{m-1}\right\rfloor}-\right.$ $1\}$, there exists $\omega_{1}, \ldots, \omega_{N} \in \overline{\mathbb{Z}}$, all of norm $\sqrt{q}, v_{1}, \ldots, v_{N} \in \mathbb{Z}$ and an integer $s \geq 0$ such that for all $n \equiv \bar{n}\left(\bmod p^{1+\left\lfloor\log _{p} l_{m-1}\right\rfloor}\right)$ with $n \geq l_{m-1}$ one has

$$
F_{q}\left(n, t_{l_{0}}, \ldots, t_{l_{m-1}}\right)=\frac{1}{q^{m}}\left(q^{n}+\frac{1}{q^{s}} \sum_{i=1}^{N} v_{i} \alpha_{i}^{n}\right)=q^{n-m}+O\left(q^{n / 2}\right) .
$$

Note that the sum is missing the factor $\frac{q-1}{p-1}$ from Theorem 1, which will be avoidable should Problem 6 be solved. Also note that it may always be possible to set $s=0$.

Problem 10: Should sufficiently general versions of Problems 1, 4 and 9 be resolved positively and bounds on the genera of the resulting curves be sufficiently small, then can one prove interesting existence results - in the best case with up to $\lfloor(1 / 2-\epsilon) n\rfloor$ coefficients prescribed for any $\epsilon>0$ - when $n$ is sufficiently large for the main term to dominate the error term in Conjecture 1 ?

\section{Acknowledgements}

This work was supported by the Swiss National Science Foundation via grant number 200021-156420, while the author was based in the Laboratory for Cryptologic Algorithms, École polytechnique fédérale de Lausanne, Switzerland. Some of the ideas were developed while the author was visiting Steven Galbraith at The University of Auckland in April 2016; I would like to thank him for his excellent hospitality and for some enlightening discussions on intersections. I would also like to thank: Wouter Castryck and Jan Tuitman for explaining to me the state-of-the-art in $p$-adic point counting techniques for curves; Daniel Panario for his useful comments on an early draft of this work; Alan Lauder for answering my questions regarding his and Wan's algorithm; Claus Diem and Benjamin Wesolowski for discussions; and Omran Ahmadi for providing the translation of Lemma 5. Finally, I would very much like to thank Thorsten Kleinjung for numerous very helpful discussions and suggestions, and for acting as a sounding board throughout much of this work.

\section{References}

1. Maple 18. Maplesoft, a division of Waterloo Maple Inc., Waterloo, Ontario.

2. Omran Ahmadi. Simplifying the Computation of Zeta Functions of Artin-Schreier Curves. Personal communication, 2017. 
3. Omran Ahmadi, Faruk Göloğlu, Robert Granger, Gary McGuire, and Emrah Sercan Yilmaz. Fibre products of supersingular curves and the enumeration of irreducible polynomials with prescribed coefficients. Finite Fields and Their Applications, 42:128 - 164, 2016.

4. Omran Ahmadi and Robert Granger. An efficient deterministic test for kloosterman sum zeros. Math. Comput., 83(285):347-363, 2014.

5. Wieb Bosma, John Cannon, and Catherine Playoust. The Magma algebra system. I. The user language. J. Symbolic Comput., 24(3-4):235-265, 1997. Computational algebra and number theory (London, 1993).

6. Jean Bourgain. Prescribing the binary digits of primes, II. Israel Journal of Mathematics, 206(1):165-182, 2015.

7. L. Carlitz. A theorem of Dickson on irreducible polynomials. Proceedings of the American Mathematical Society, 3:693$700,1952$.

8. K. Cattell, C.R. Miers, F. Ruskey, M. Serra, and J. Sawada. The number of irreducible polynomials over GF(2) with given trace and subtrace. J. Combin. Math. Combin. Comput., 47:31 - 64, 2003.

9. Pascale Charpin and Guang Gong. Hyperbent functions, Kloosterman sums, and Dickson polynomials. IEEE Trans. Inform. Theory, 54(9):4230-4238, 2008.

10. Stephen D. Cohen. Uniform distribution of polynomials over finite fields. Journal of the London Mathematical Society, s2-6(1):93-102, 1972.

11. Stephen D. Cohen. Primitive Polynomials over Small Fields, pages 197-214. Springer Berlin Heidelberg, Berlin, Heidelberg, 2004.

12. John Dalbec. Multisymmetric functions. Beitrge zur Algebra und Geometrie, 40(1):27-51, 1999.

13. John. F. Dillon. Elementary Hadamard Difference Sets. PhD Thesis. University of Maryland, 1974.

14. Marcus du Sautoy. Counting p-groups and nilpotent groups. Publications Mathématiques de l'Institut des Hautes Études Scientifiques, 92(1):63-112, 2000.

15. Marcus du Sautoy. Counting subgroups in nilpotent groups and points on elliptic curves. Journal für die reine und angewandte Mathematik, 549:1-21, 2002.

16. Shuqin Fan and Wenbao Han. p-adic formal series and primitive polynomials over finite fields. Proc. Amer. Math. Soc., 132(1):15-31 (electronic), 2004.

17. Robert W. Fitzgerald and Joseph L. Yucas. Irreducible polynomials over $G F(2)$ with three prescribed coefficients. Finite Fields and Their Applications, 9(3):286 - 299, 2003.

18. Kseniya Garaschuk and Petr Lisoněk. On binary Kloosterman sums divisible by 3. Des. Codes Cryptogr., 49(1-3):347-357, 2008.

19. C.F. Gauss. Untersuchungen Uber Höhere Arithmetik (second edition). Chelsea publishing company, New York, 1981.

20. F. Göloğlu, P. Lisoněk, G. McGuire, and R. Moloney. Binary kloosterman sums modulo 256 and coefficients of the characteristic polynomial. Information Theory, IEEE Transactions on, 58(4):2516-2523, 2012.

21. Faruk Göloglu, Gary McGuire, and Richard Moloney. Ternary kloosterman sums modulo 18 using stickelberger's theorem. In SETA, pages 196-203, 2010.

22. Faruk Göloğlu, Gary McGuire, and Richard Moloney. Binary Kloosterman sums using Stickelberger's theorem and the Gross-Koblitz formula. Acta Arith., 148(3):269-279, 2011.

23. Junsoo Ha. Irreducible polynomials with several prescribed coefficients. Finite Fields and Their Applications, 40:10 - 25, 2016.

24. J. Hadamard. Résolution d'une question relative aux déterminants. Bulletin des Sciences Mathématiques, 17:240-246, 1893.

25. David R. Hayes. The distribution of irreducibles in GF[ q,x ]. Transactions of the American Mathematical Society, 117:101-127, 1965.

26. Tor Helleseth and Victor Zinoviev. On $Z_{4}$-linear Goethals codes and Kloosterman sums. Des. Codes Cryptogr., 17(13):269-288, 1999.

27. Chih-Nung Hsu. The Distribution of Irreducible Polynomials in $\mathbb{F}_{q}[t]$. Journal of Number Theory, 61(1):85 - 96, 1996.

28. E. Kani and M. Rosen. Idempotent relations and factors of jacobians. Mathematische Annalen, 284(2):307-327, 1989.

29. Nicholas Katz and Ron Livné. Sommes de Kloosterman et courbes elliptiques universelles en caractéristiques 2 et 3 . $C$. R. Acad. Sci. Paris Sér. I Math., 309(11):723-726, 1989.

30. Behzad Omidi Koma and Daniel Panario. Approximating the Number of Irreducible Polynomials over GF(2) with Several Prescribed Coefficients. Journal of Combinatorial Mathematics and Combinatorial Computing, 90:255-284, 2014.

31. B.O. Koma. The Number of Irreducible Polynomials Over a Finite Field With Prescribed Coefficients. PhD thesis, Carleton University, 2010.

32. E. N. Kuz'min. A class of irreducible polynomials over a finite field. (Russian) Dokl. Akad. Nauk SSSR, 313(3):552-555, 1990. translation in Soviet Math. Dokl. 42 (1991), no. 1, 45-48.

33. E. N. Kuz'min. Irreducible polynomials over a finite field and an analogue of Gauss sums over a field of characteristic 2. Siberian Mathematical Journal, 32(6):982-989, 1991.

34. Gilles Lachaud and Jacques Wolfmann. The weights of the orthogonals of the extended quadratic binary Goppa codes. IEEE Trans. Inform. Theory, 36(3):686-692, 1990.

35. Alan G. B. Lauder and Daqing Wan. Computing zeta functions of Artin-Schreier curves over finite fields. LMS Journal of Computation and Mathematics, 5:3455, 2002.

36. Rudolf Lidl and Harald Niederreiter. Finite fields, volume 20 of Encyclopedia of Mathematics and its Applications. Cambridge University Press, Cambridge, second edition, 1997. 
37. Petr Lisoněk. On the connection between Kloosterman sums and elliptic curves. In Sequences and their applicationsSETA 2008, volume 5203 of Lecture Notes in Comput. Sci., pages 182-187. Springer, Berlin, 2008.

38. Petr Lisoněk and Marko Moisio. On zeros of kloosterman sums. Designs, Codes and Cryptography, 59:223-230, 2011. 10.1007/s10623-010-9457-x.

39. C. Robert Miers and Frank Ruskey. COUNTING STRINGS WITH GIVEN ELEMENTARY SYMMETRIC FUNCTION EVALUATIONS I: STRINGS OVER $\mathbb{Z}_{p}$ WITH $p$ PRIME. SIAM J. Discrete Math., 17(4):675-685, 2004.

40. C. Robert Miers and Frank Ruskey. COUNTING STRINGS WITH GIVEN ELEMENTARY SYMMETRIC FUNCTION EVALUATIONS II: CIRCULAR STRINGS. SIAM J. Discrete Math., 18(1):71-82, 2004.

41. Marko Moisio. The divisibility modulo 24 of Kloosterman sums on $\mathrm{GF}\left(2^{m}\right), m$ even. Finite Fields Appl., 15(2):174-184, 2009.

42. Marko Moisio and Kalle Ranto. Kloosterman sum identities and low-weight codewords in a cyclic code with two zeros. Finite Fields Appl., 13(4):922-935, 2007.

43. Daniel Panario. Open Problems for Polynomials over Finite Fields and Applications. In Çetin Kaya Koç, editor, Open Problems in Mathematics and Computational Science, chapter 5, pages 111-126. Springer Publishing Company, Incorporated, 2015.

44. Paul Pollack. Irreducible polynomials with several prescribed coefficients. Finite Fields and Their Applications, 22:70 78, 2013.

45. Won-Ho Ri, Gum-Chol Myong, Ryul Kim, and Chang-Il Rim. The number of irreducible polynomials over finite fields of characteristic 2 with given trace and subtrace. Finite Fields and Their Applications, 29(0):118 - 131, 2014.

46. P. L. Sharma, M. Rehan, and S. Sharma. Counting irreducible polynomials over GF(3) with first and third coefficients given. Asian-European Journal of Mathematics, 08(01):1550015, 2015.

47. Henning Stichtenoth and Chaoping Xing. On the structure of the divisor class group of a class of curves over finite fields. Archiv der Mathematik, 65(2):141-150, 1995.

48. J. J. Sylvester. Thoughts on Orthogonal Matrices, Simultaneous Sign-Successions, and Tessellated Pavements in Two or More Colours, with Applications to Newtons Rule, Ornamental Tile-Work, and the Theory of Numbers. Phil. Mag., 34:461-475, 1867.

49. Jan Tuitman. Counting points on curves using a map to $\mathbf{P}^{1}$. Math. Comp., 85:961-981, 2016.

50. Jan Tuitman. Counting points on curves using a map to $\mathbf{P}^{1}$, II. Finite Fields and Their Applications, 45:301 - 322, 2017.

51. Gerard van der Geer and Marcel van der Vlugt. Kloosterman sums and the p-torsion of certain Jacobians. Math. Ann., 290(3):549-563, 1991.

52. Gerard van der Geer and Marcel van der Vlugt. Fibre Products of Artin-Schreier Curves and Generalized Hamming Weights of Codes. Journal of Combinatorial Theory, Series A, 70(2):337 - 348, 1995.

53. José Felipe Voloch. Generators of finite fields with powers of trace zero and cyclotomic function fields. Portugal. Math. (N.S.), 73(1):65-70, 2016.

54. J.L. Walsh. A closed set of normal orthogonal functions. Amer. J. Math, 45:5-24, 1923.

55. Han W.B. On Cohens Problem. Chinacrypt96, Academic Press, pages 231-235, 1996 (in Chinese).

56. A. Weil. Sur les courbes algébriques et les variétés qui sen déduisent,. Actualités Sci. Ind. = Publ. Inst. Math. Univ. Strasbourg 7 (1945). Hermann et Cie., Paris, 1041, 1948.

57. Joseph L. Yucas and Gary L. Mullen. Irreducible polynomials over $G F(2)$ with prescribed coefficients. Discrete Mathematics, 274(13):265 - 279, 2004.

58. S. Zabek. Sur la periodicite modulo m des suits de nombres $\left(\begin{array}{l}n \\ k\end{array}\right)$. Ann. Univ. Mariae Curie-Sklodowska, 10(Sect. A):37-47, 1956. 